\title{
Cooperative genetic networks drive a mammalian cell
}

\section{state transition}

Andreas Lackner ${ }^{\# 1}$, Robert Sehlke ${ }^{\# 2}$, Marius Garmhausen ${ }^{\# 2}$, Giuliano Giuseppe Stirparo ${ }^{\# 3}$, Michelle Huth $\$ 1$, Fabian Titz-Teixeira ${ }^{\$ 2}$, Petra van der Lelij ${ }^{1}$, Julia Ramesmayer ${ }^{1}$, Henry Fabian Thomas $^{1}$, Meryem Ralser ${ }^{3}$, Laura Santini ${ }^{1}$, Mihail Sarov ${ }^{4}$, A. Francis Stewart ${ }^{4,5}$, Austin Smith ${ }^{3}$, Andreas Beyer*2, Martin Leeb*1

\footnotetext{
${ }^{1}$ Max Perutz Laboratories Vienna, University of Vienna, Vienna Biocenter, Dr Bohr Gasse 9, 1030 Vienna

${ }^{2}$ CECAD, Faculty of Mathematics and Natural Sciences, Medical Faculty Cologne, University of Cologne, Joseph-
} Stelzmann-Str. 2650931 Köln, Germany

${ }^{3}$ Wellcome - MRC Cambridge Stem Cell Institute, University of Cambridge, Cambridge CB2 OAW, UK

${ }^{4}$ Max Planck Institute of Molecular Cell Biology and Genetics, Pfotenhauerstraße 108

01307 Dresden, Germany

${ }^{5}$ Biotechnology Center, Center for Molecular and Cellular Bioengineering, Technische Universität Dresden, Tatzberg 47, 01307 Dresden, Germany

${ }^{6}$ Center for Molecular Medicine (CMMC), University of Cologne, Cologne, Germany.

\# Equal contribution

\$ Equal contribution

* Corresponding authors

\section{Key words:}

Haploid ES Cells, Systems Biology, Knock Out, Pluripotency, Naïve to Formative Transition, Epiblast, Signalling, Exit from Naïve Pluripotency, Gene Regulatory Networks 


\section{Abstract}

In the mammalian embryo, epiblast cells must exit their naïve state and acquire formative pluripotency. This cell state transition is recapitulated by mouse embryonic stem cells (ESCs), which undergo pluripotency progression in defined conditions in vitro. However, our understanding of the molecular cascades and gene-networks involved in the exit from naïve pluripotency remains fragmented. Here we employed a combination of genetic screens in haploid ESCs, CRISPR/Cas9 gene disruption, large-scale transcriptomics and computational systems-biology to delineate the regulatory circuits governing naïve state exit. Transcriptome profiles for 73 knockout ESC lines predominantly manifest delays on the trajectory from naive to formative epiblast. We find that gene networks operative in ESCs are active during transition from pre- to post-implantation epiblast in utero. We identified 374 naïve-associated genes tightly connected to epiblast state and largely conserved in human ESCs and primate embryos. Integrated analysis of mutant transcriptomes revealed funneling of multiple gene activities into discrete regulatory modules. Finally, we delineate how intersections with signaling pathways direct this pivotal mammalian cell state transition. 


\section{Introduction}

2 Mouse embryonic stem cells (ESCs) can self-renew in defined conditions in a ground state of

3 naïve pluripotency ${ }^{1}$. The ESC exit from naïve pluripotency provides an amenable

4 experimental system for dissection of a cell fate decision paradigm ${ }^{2,3}$. Naïve pluripotency is

5 under control of a gene regulatory network (GRN) containing the core pluripotency

6 transcription factors (TFs) Pou5f1, Sox2 and naïve-specific TFs like Nanog, Esrrb, Klf4 and

7 others ${ }^{4-6}$. In defined cell culture conditions that include inhibitors against Mek1/2

8 (PD0325901) and Gsk3 (CHIR990201, CH; collectively termed '2i'), ESCs can be homogenously

9 maintained in the naïve state ${ }^{7}$. Within 24 to 36 hours after withdrawal of 2i, ESCs transit into

10 formative pluripotency, entirely losing naïve identity ${ }^{2}$. During this transition, the naïve GRN

11 is extinguished and expression of formative factors like Otx2, Pou3f1, Dnmt3a/b and Fgf5 is

12 initiated. A similar transition is evident during peri-implantation development, where the TF-

13 network maintaining naïve pluripotency dissolves between embryonic day (E) 4.5 and E5.5 ${ }^{8-}$

1410.

15 The speed of the naïve to formative GRN transition is notable because: i) the cell cycle is

16 around 12 hours long; ii) all factors that are required to establish and maintain naïve

17 pluripotency are expressed robustly in naïve cells; and (iii) the naïve pluripotency network is

18 recursively self-reinforcing. The rapid dissolution of naïve pluripotency implies the existence

19 of circuit-breaking mechanisms. In recent years, we and others have identified various factors promoting ESC differentiation using screens in haploid and diploid ES cells ${ }^{11-15}$. 
21 Robust assays employing ESCs expressing a Rex1-promoter driven destabilised GFP reporter ESC line (Rex1::GFPd2) enable the dissection of the exit from naïve pluripotency in high resolution ${ }^{2,16}$. Rex1-GFP downregulation is initiated within $24 \mathrm{~h}$ after $2 \mathrm{i}$ withdrawal (N24) and completed after $48 \mathrm{~h}$ (N48). Nevertheless, the exact nature, mechanistic underpinnings and sequence of events during exit from naïve pluripotency remain only partially understood. In particular, we lack insight into how the different molecular components of the system cooperate to elicit proper cell fate transition.

Here, we have driven a Rex1-GFP reporter screen to saturation, thus providing an extensive list of genes and pathways involved in the exit from naïve pluripotency. We utilized this information in a systems biology approach to explore regulatory principles of the exit from naïve pluripotency. To evaluate dependencies and causal relationships within the pluripotency and differentiation circuitries, we probed the response of the differentiation program to a comprehensive series of exit factor gene knockouts. Through computational integration of molecular profiling data with regulatory networks and in vivo GRN-trajectories, we expose the regulatory foundations of a cell fate choice paradigm at a pivotal junction in early mammalian development. 


\section{Results}

\section{Haploid ES cell saturation screen}

Haploid ES cells are an efficient platform for insertional mutagenesis-based screens ${ }^{11,17,18 .}$

We previously reported a medium-scale screen comprising approximately $5 \times 10^{4}$ mutagenic events to identify factors regulating the exit from naïve pluripotency ${ }^{12}$. We have now driven this approach to saturation by assaying approximately 1.2 million mutations in receptive genomic regions that cause delays in Rex1 downregulation in two independent Rex1-reporter cell lines (Figure 1ab), utilizing three different mutagenic vectors in 35 independent screens (Figure 1cd).

47 Stringent filtering resulted in a candidate list comprising 489 genes (Supplementary Table 1). Reassuringly, the known exit from naïve pluripotency regulators Tcf7l1, Fgfr2, Jarid2 and Mapk1 (Erk2) ${ }^{19}$ were among the highest ranked (Supplementary Figure 1a). The candidate hit genes were enriched for processes involved in transcription regulation, epigenetics and signalling-related functions (Supplementary Figures 1bc; Supplementary Table 1), as well as RNA binding functions in line with emerging evidence of RNA regulatory mechanisms in cell fate control ${ }^{20}$.

These screens generated a candidate inventory of the machinery that mediates exit from naïve pluripotency. Many identified genes are not specific to pluripotency and have functions

57 in common pathways and processes, implying that the exit from naïve pluripotency utilizes 
widely expressed cellular machinery. Therefore, mechanisms mediating ESC transition might also be utilised in other differentiation processes.

\section{Establishment of a mutant ESC library for systematic transcriptional profiling}

To characterise deficiencies in naïve exit, we generated KO ESC lines deficient for 73 selected genes, comprising top ranked genes from the mutagenic screen together with components from pathways and protein complexes for which multiple members were recovered, even if just below the cut-off threshold (e.g. the Paf complex member Leo1, the mTORC1 regulator Tsc2 and the NMD component Smg6), and Mbd3, Zfp281 and L3mbt/3 as known players in the exit from naïve pluripotency ${ }^{14}$. Three control genes were included that are either not expressed in ES cells (Nestin), expected to be neutral (Hprt), or whose ablation was expected to accelerate differentiation (cMyc). Paired gRNAs were used to disrupt target genes in a

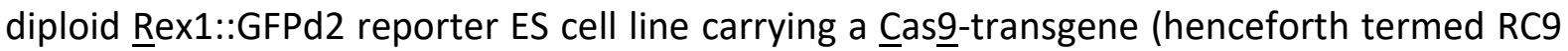
cells) (Figure 1a). Following an efficient parallelized approach, we established passage matched and isogenic cell lines, thus maximising comparability (Supplementary Figure 1de).

Full protein deficiency was validated for 14 KOs (Eed, Suz12, Jarid2, Kdm6a, Smg5, Smg6, Smg7, Tsc2, Pten, Raf1, Tcf7l1, Leo1, Nmt1, and Csnk1a1), while reduced protein expression in concordance with a heterozygote phenotype was confirmed for Mapk1 (Supplementary Figure 1f). For five further genes (Alg13, Dido1, Msi2, Etv5, Jmjd1c) we confirmed absence of the corresponding transcripts or specific out of frame deletion of an exon by RT-qPCR or Sanger sequencing of RT-PCR products. Successful rescue experiments using 3xflag-tagged transgenes for six genes (Rbpj, Etv5, Fgfr1, Jarid2, Mbd3, and Tcf7l1) established causality 
knockouts showed the expected impact on RNA or protein expression. However, we cannot exclude the possibility of hypomorphic phenotypes in some cases.

83

ESC differentiation behaviour is highly dependent on cell density and timing of medium changes. To enable robust comparison of the differentiation of multiple KO ESC lines in parallel, we performed differentiations batch wise in duplicates, always including WT ESCS and negative controls across seven experiments. At N24 we assayed the differentiation status by FACS analysis (Figure 1e) and extracted RNA for transcriptome analysis.

\section{The exit machinery is already poised in $\mathbf{2} i$}

Batch corrected RNA-seq data comprising 14 replicates of WT ES cells indicated 3058 differentially expressed genes (DEG) between $2 \mathrm{i}$ and N24 (HO: $|\mathrm{FC}|<1.5$, FDR $\leq 0.05$; Figure genes identified in the haploid screens including the 73 genes selected for KO did not change significantly in transcript expression between $2 \mathrm{i}$ and N24 and were not present in the list of DEG (Figures $1 f$ and S1K), with only Jarid2, L3mbt/3, Pten and Zfp423 showing upregulation at N24 (FC $\geq 2$; adj.p. $\leq 0.05$ ). This implies that the exit machinery is already embedded in the ground state and ESCs are poised for rapid decommissioning of naïve identity and entry into differentiation ${ }^{19}$.

To facilitate interrogation of the KO gene expression datasets, we developed an interactive

101 online tool (GENEES - Genetic Network Explorer for Embryonic Stem Cells - 
Using t-Distributed Stochastic Neighbour Embedding (t-SNE), we visualized similarities

between KOs based on expression of the DEG in $2 \mathrm{i}$ and at N24. We observed clustering of

(Fgf/ERK), Smg5-Smg6-Smg7 (NMD; nonsense mediated decay), Mta3-Mbd3 (NuRD), Ptenculture condition (2i and N24) and to a lesser extent by genotype (Supplementary Figure 1i).

111 This is consistent with the phenotypic observation that despite manifesting differentiation

112 delays at $\mathrm{N} 24$, all of the KO ESCs ultimately departed from the naïve state during longer differentiation timecourses, as measured by loss of Rex1GFP. Furthermore, even KOs that showed extensive Rex1GFP downregulation delays at N24 displayed transcription profiles that were globally adjusted towards differentiation. Therefore, the knockout of a single gene is not sufficient to permanently block exit from naïve pluripotency in culture, in accord with the finding that ternary depletion of $T c f 7 \mid 1, E t v 5$ and $R b p j$ is required for sustained selfrenewal in the absence of $2 \mathrm{i}$ or LIF ${ }^{21}$.

\section{The exceptional role of $\operatorname{Csnk1a1}$ and the involvement of compensatory mechanisms}

121 The Csnk1a1 KO is an exception to this generalization, clustering with $2 \mathrm{i}$ samples at N24

122 (Supplementary Figure 1i). Csnk1a1 is a serine threonine kinase and a component of the beta123 catenin destruction complex. Although KO of another destruction complex member Apc, or 124 of the downstream repressor $T c f 7 / 1$ resulted in the upregulation of similar gene-sets 125 (Supplementary Figure 1I), we observed stronger differentiation defects and larger amplitude of gene deregulation in two independently derived Csnk1a1 mutants. However, these mutants also exhibited markedly reduced population doubling rates, which was not shown by 
Apc or $T c f 711$ mutants. Upon continuous culture in $2 \mathrm{i}$ medium ( $\sim 5$ passages), proliferation was restored in Csnk1a1 KO cells and differentiation potential was regained, suggesting upregulation of compensatory mechanisms and a likely effect of proliferation rate on differentiation kinetics. Csnk1a1 can be chemically inactivated using Epiblastin $A,{ }^{22}$. We found that Epiblastin A partially blocked exit from naïve pluripotency, without affecting proliferation within the duration of the assay (Supplementary Figure $1 \mathrm{~m}$ ). A second case of phenotype adaptation was observed with Pum1. Pum1 KOs showed pronounced differentiation delays during early passages (Supplementary Figure 1n), as also seen for acute Pum1 depletion by siRNA and in previously generated CRISPR KO ESCs ${ }^{12}$. However, the phenotype was lost in later passages and Pum1 KO cells showed WT like expression-levels of naïve marker genes (Figures 2a and S1a).

\section{Robust feedback wiring in the naïve TF network}

141 Interestingly, the transcriptome data revealed that exit factors do not, in general, reduce naïve transcription factor (TF) expression in the ground state (Supplementary Figure 2a). $R b p j$ and Trim71 $\mathrm{KO}$ resulted in moderate increases in Klf4, while the aforementioned Csnk1a1 KO

144 ESCs showed limited up-regulation of both KIf4 and Tbx3 in 2i. Other KOs had only minor

145 effects on the naïve network in 2i. These data are consistent with robust feedback wiring in the naïve TF network ${ }^{4,5}$ and neutralisation of most 'differentiation drivers' in $2 \mathrm{i}$ in culture ${ }^{23}$.

147 In contrast, we observed a more extensive impact on formative markers. Several KO cell lines 148 showed lower baseline expression in $2 \mathrm{i}$ of Otx2, Fgf5, Dnmt3a/b and Pou3f1 (Oct6)

149 (Supplementary Figure 2b). In line with recent results, we noted that depletion of several 150 Fgf/ERK components resulted in reduced Dnmt3a/b, Pou3f1 and Fgf5 expression in $2 \mathrm{i}^{21}$. 151 Although Fgf/ERK signalling is effectively inhibited in $2 \mathrm{i}^{7}$, our data suggest that either residual 
pathway activity or potential moonlighting functions of pathway components mediate poised expression of the formative pluripotency programme in $2 \mathrm{i}$.

Clustering based on the expression of ten naïve pluripotency marker genes showed that the downregulation of the naïve pluripotency TFs during formative differentiation is defective across multiple KOs (Figure 2a). Although expression of the naïve TFs was highly correlated, KIf2 appeared to be an exception. Klf2 downregulation was notably impaired in Tcf7l1 KO ESCs, whereas it was unaffected by several KOs, including Jarid2, despite a comparable extent of deregulation of most other naïve marker genes. This indicates that Klf2 expression can be uncoupled from the core naïve network. Forced $K l f 2$ expression stabilizes self-renewal ${ }^{24,25}$. KIf2 depletion destabilizes mouse ESC identity ${ }^{26}$ and increases the speed of Rex1-GFP downregulation upon 2i withdrawal (Supplementary Figure 2c). The differentiation delay in Tcf7l1 KO ES cells is partially dependent on Klf2 (Figure 2b), consistent with direct regulation that separable gene-networks contribute to dismantling naïve pluripotency.

\section{Effects of exit gene depletion on global gene regulation}

168 We used two measures to gauge differentiation delays in KOs compared to WT cells: first, the average expression levels of a set of naïve marker genes (defined throughout this manuscript

170 as the average deregulation of the naïve marker genes Esrrb, Nanog, Tfcp2/1, Tbx3, Prdm14

171 and KIf4 at N24); second, the extent of global transcriptome adjustments between 2i and N24.

172 Generally, the delayed extinction of the naïve TF network in KOs at N24 was accompanied by

173 reduced gene expression change between $2 i$ and N24 ( $R=-0.91$; Figure $2 c)$. However, some

174 KOs deviated from this pattern. Alg13, Jmjd1c and Jarid2 mutants showed a less pronounced 
change in naïve marker gene down-regulation than expected based on the reduced level of global gene expression adjustments towards an N24 profile. Trim71 KO cells in contrast displayed modest global transcription changes but a more evident effect on naïve TF expression levels. Thus, the RNA binding protein Trim71 appears to be focused on the regulation of naïve pluripotency TF genes, although the ensuing exit delay is modest.

A general correlation was observed between the numbers of genes deregulated in $2 \mathrm{i}$ and at

N24. Fgfr1 and Tcf7l1 KOs were two exceptions to this correlation with relatively few genes in $2 \mathrm{i}$ is largely masked because they are in the same pathways as the inhibitor targets Mek1/2 (PD0325901) and Gsk3 (Chiron 990201) ${ }^{7,23}$. Defective differentiation could simply equate with the extent of overall gene deregulation. We therefore mapped the average deregulation of naïve marker genes onto Figure $2 \mathrm{~d}$. Most KOs showing the strongest deregulation of naïve marker genes correlated with large-scale gene deregulation (both in $2 \mathrm{i}$ and at N24). However, there were several exceptions: for example, KO of Eed, Suz12, Trim71, and Ctbp2 affected a substantial number of genes, but caused naïve marker deregulation that was weaker than expected assuming a direct correlation between the number of deregulated genes and the differentiation phenotype. Vice versa, KO of $M b d 3, C d k 8$ or Pten showed marked naïve marker downregulation delays, despite a relatively mild impact on overall transcription 
Transcriptome analysis reveals a genetic interaction between Jmjd1c and Tcf7l1

The putative histone 3 lysine 9 (H3K9) demethylase $J m j d 1 c$ (Kdm3c) KO is a case of particular note. Few genes were significantly deregulated at N24 (Figure 2d), with a mild global neutralisation of differentiation-induced transcriptome changes (Figure 2c). Neither the role of Jmjd1c in pluripotency regulation, nor its mode of action are known. We used multiple regression analysis to determine the similarity of the Jmjd1c KO to all other KO RNA-seq profiles. At N24, the Jmjd1c KO transcriptome was most similar to the Tcf7l1 $\mathrm{KO}$ profiles (Supplementary Figure 2e), suggesting a potential functional connection between Jmjd1c and $T c f 7 \mid 1$. Indeed, Jmjd1c has recently been reported as a high-confidence protein-interactor of Tcf7l1 ${ }^{28}$. The $2 \mathrm{i}$ component Chiron 99021 is a specific inhibitor of Gsk3 and phenocopies deletion of $T c f 711$, which is the downstream repressor in ESCs ${ }^{29,}{ }^{30}$. Accordingly, Chiron 99021 delays the differentiation of WT ESCs, but had little additional effect on Tcf7/1 KOs (Figure 2e). Jmjd1c deficient ESCs did not show a discernible phenotype at N72, in line with minimal deregulation of the naïve TF network. Addition of Chiron 99021 had a stronger than expected effect on Jmjd1c KOs and resulted in a synthetic enhanced delay phenotype (Figure 2e), suggesting a cooperative activity of Jmjd1c and the Wnt/Tcf7l1 axis in the exit from naïve pluripotency.

\section{Relative quantification of differentiation delays in vitro}

217 To quantify differentiation delays, we obtained RNA-seq data from a $2 \mathrm{~h}$-resolved WT ESC differentiation time course (Figures 3a and Supplementary Figure 3a). We then compared the expression patterns of specific gene-sets in a given $\mathrm{KO}$ at $\mathrm{N} 24$ to the expression of the same gene-set along the WT $2 \mathrm{i}$ to N32 trajectory. This enabled us to 'position' each KO along the

221 trajectory and thus quantify the differentiation delay with a precision of about 4 h (Figure $3 b$, 
Supplementary Table 2). In a complementary approach, we used all 3058 DEG between 2i and

N24 as an alternative reference set. This yielded similar, but on average slightly less pronounced differentiation delays (Figures $3 c$ and Supplementary Figure 3b), supporting the hypothesis that the naïve TF network is regulated in concordance with but partially independently from the rest of the transcriptome during exit from naïve pluripotency.

The regulatory program for pre- to post-implantation epiblast transition is preserved in

most mutants

To explore the extent to which our in vitro data captures in vivo regulation, we first compared global transcriptomes of WT and KO ESCs to single cell transcriptome data from the in vivo pre- (E4.5) and post-implantation (E5.5 and E6.5) mouse epiblast ${ }^{10}$ (Figure 4a,B). As expected

${ }^{8}$, WT and KO cells in $2 \mathrm{i}$ showed greater similarity (fraction of identity - FOI) to the E4.5 than to the E5.5 epiblast. Cells at N24, in contrast, showed higher FOI to the E5.5 epiblast. Cells in

$2 \mathrm{i}$ and at $\mathrm{N} 24$ showed low FOI to E6.5 epiblast, underscoring that $24 \mathrm{~h} 2 \mathrm{i}$ withdrawal models the E4.5 to E5.5 epiblast transition ${ }^{2}$. Interestingly, some KO ESCs showed expression features in $2 \mathrm{i}$ with increased similarity to the in vivo epiblast (Figures $4 \mathrm{c}$, d and Supplementary Table 3). Among those were KOs showing strong in vitro differentiation defects, such as Zfp281, Tsc2 and Etv5, but also Trim71 and Smg7, which showed only a modest differentiation delay in culture.

241 At N24 several KOs, such as Zfp281, Tsc2, Smg5 and Smg6, retained strong similarity to the 242 E4.5 epiblast (Figures 4c,e and Supplementary Figure 4a). Strikingly, Zfp281 KO profiles at N24 243 showed similarity to the E4.5 epiblast on par with WT cells cultured in $2 \mathrm{i}$ (Figures $4 \mathrm{c}$ and 244 Supplementary Figure 4a), consistent with an overt differentiation delay phenotype ${ }^{31}$. 
Overall, there was a good correlation between the in vitro differentiation delay and the similarity with pre-implantation epiblast cells: the N24 KO transcriptomes that were more similar to the E4.5 epiblast exhibited stronger exit-delay phenotypes (Figure 4e, regulated by similar mechanisms during in vivo and in vitro transitions to formative pluripotency. However, at N24 three KOs (Jarid2, Tcf7l1, and Trp53) showed a similarity to the E4.5 epiblast which was smaller than expected based on their strong in vitro differentiation defects (Figures $2 \mathrm{a}$ and $4 \mathrm{e}$ ).

We then performed principal component analysis (PCA) for genes variably expressed in embryo development, using averaged values for inner cell mass (ICM), primitive endoderm (PrE) and E4.5, 5.5 and 6.5 epiblast ${ }^{10}$. The PCA separated the $2 \mathrm{i}$ and N24 transcriptomes into two clusters with proximity to PrE/EPI and E5.5 respectively. Notably, in vitro differentiation defects were reflected by closer proximity of mutant N24 profiles to the E4.5 epiblast (Figure 4f). Overlaying the PCA with a colour gradient indicating similarity to PrE shows that multiple KOs in 2i gained similarity to $\operatorname{PrE}$ (Supplementary Figure 4d). Concordantly, the expression of Eed, Suz12, Zfp423 and Csnk1a1 KOs in 2i (Supplementary Figure 4e). A shift toward PrEidentity was most pronounced for the PRC2 KOs Eed and Suz12. Notably, this group of KOs (Csnk1a1, PRC2, Zfp423, which exhibited substantial in vitro differentiation defects while showing reduced similarity to the E4.5 epiblast, also showed decreased similarity between their N24 profiles and the E5.5 epiblast (Supplementary Figure 4d). We therefore suggest that deregulation of the PrE-programme above a certain threshold has disengaged these KOs from 
similarity to the E4.5 in $2 \mathrm{i}$ and decreased similarity to the E5.5 epiblast at N24 are deficient for genes regulating the formative transition during development (e.g. Zfp281, Etv5, Rbpj and,

271 Trim71, Supplementary Figure 4d).

272

\section{Correspondence to primate embryogenesis}

274 Despite morphological and timing differences between rodent and primate peri-implantation 275 development, embryos of both orders appear to transit through similar pluripotency states ${ }^{1,}$ 32. To examine this issue, we compared transcriptional profiles of our KO series and cells of

277 the macaque in vivo naive- and post-implantation epiblast ${ }^{33}$. In general, ESCs in $2 \mathrm{i}$ were more similar than cells at $\mathrm{N} 24$ to the pre-implantation macaque epiblast (Figure $4 \mathrm{~g}$ ). Correspondingly, N24 cells were closer to the macaque post implantation epiblast. Interestingly, KOs showing higher identity with the mouse in vivo epiblast also displayed an increased similarity with the macaque pre-implantation epiblast (Supplementary Figures $4 \mathrm{f}, \mathrm{h})$. We also compared our datasets to data obtained from in vitro capacitation during human naïve to primed ESC differentiation ${ }^{34}$. KOs displaying strong differentiation defects in mouse cell culture had higher correspondence to naïve human ESCs and, at N24, lower observations suggest that the overall GRN-redeployment during the naïve to formative transition is conserved between rodents and primates.

\section{Identification of an extended naïve pluripotency network}

289 ESC differentiation requires fine-tuned coordination between extinction of the naïve and initiation of the formative transcription networks. To date, only incomplete inventories of the 
defined based simply on differential expression between $2 \mathrm{i}$ and N24, because not all DEG will be functionally linked to the naïve GRN. To identify those genes that show specific linkage to the core naïve pluripotency network, we trained regression models to predict expression changes across all KOs (in $2 \mathrm{i}$ and at N24) as a function of naïve marker log-fold-changes. The 374 genes whose expression was tightly correlated $\left(R^{2}>0.7\right)$ with one or several of the seven core pluripotency markers (Nanog, Esrrb, Tbx3, Tfcp2I1, KIf4, Prdm14 and Zfp42) were defined as 'Naive-Associated Genes' (NAGs; Supplementary Table 3). The naïve pluripotency TFs not represented in the defining TF-set were correctly identified as NAGs, including Klf5, NrOb1 and $N r 5 a 2$. However, $K I f 2$ is not one of the NAGs being only weakly associated $\left(R^{2}=0.53\right)$ with the naïve network. This concords with our earlier observation that $K l f 2$ expression can be uncoupled from the naïve TF network. Of further note, KIf2 expression is barely detectable in marmoset or human pre-implantation epiblast cells (Supplementary Figure 5b).

The NAG showing the highest correlation with the naïve core network is $P d g f a$, which is relatively highly expressed in ES cells (FPKM 70). Pdgfa has no known role in ESC selfrenewal, but functions in segregation of the primitive endoderm ${ }^{35}$. To examine whether the link between Pdgfa and the naïve transcription factor network is maintained in vivo, we utilized GRAPPA, a tool to visualize single cell expression data from preimplantation development ${ }^{36}$. Indeed, Pdgfa is uniformly expressed in the E4.5 epiblast (Supplementary

311 Figure 5c). Interestingly, its cognate receptor Pdgfra is neither expressed in ES cells nor the naïve epiblast (FPKM in WT ESCs $<0.5$ ), but specifically marks the neighbouring primitive endoderm at E4.5 ${ }^{37}$. 
We surveyed the expression of NAGs at the transition from naïve to post-implantation pluripotency in the single cell RNA-seq datasets from E4.5, E5.5 and E6.5 epiblast cells ${ }^{10}$. We detected a clear enrichment of NAGs within genes that separate the pre- from the postimplantation epiblast in the differentiation-state resolving dimension of a principal component analysis (PCA) (Figures 5a and Supplementary Figure 5d), highlighting that NAGs are a set of indicators for the naïve epiblast state in vitro and in vivo. Strikingly, NAGs showed a near identical fold change behaviour in vitro and in vivo during the epiblast transition from expressed genes in ESC differentiation in vitro. Furthermore, NAG orthologues showed significantly co-regulated expression dynamics during macaque pre- to post-implantation epiblast differentiation in vivo (Figure $5 \mathrm{c}, \mathrm{e}, \mathrm{f}$ ) and during capacitation of human ESCs in vitro (Figures $5 d, e, f)$, which further underscores their relevance. We thus propose that NAGs constitute an integral component of the naïve transcriptional network with potential functional relevance in vivo across mammalian species.

\section{Deregulation of signaling cascades is a hallmark of differentiation delay}

We next analysed the extent of deregulation of five key signalling pathways known to be in the activity of a signalling pathway are not necessarily reflected in expression changes of pathway member transcripts. Thus, in order to quantify pathway activities, we employed 'expression footprints'. We identified pathway specific marker gene-sets, each containing 50 genes, reporting pathway activity changes. These marker sets were determined using N24 
(Tcf7l1 KO), Fgf/ERK (Fgfr1 and Ptpn11 KOs), and Notch (Rbpj KO) pathway targets

(Supplementary Figure 6a, Supplementary Table 4). Overlaps with available Tcf7l1 and Rbpj

ChIP and activity profiles ${ }^{27,43}$ supported the reliability of this approach (Supplementary Figure for 24 hours. This resulted in a list of LIF-sensitive genes including known targets like Socs3, footprints present non-overlapping sets of marker genes whose expression state is indicative of the activity of the respective signaling pathway.

Using the expression footprints, we then asked whether we could detect a preferential deregulation of one or more of these signalling footprints in specific KOs either in $2 \mathrm{i}$ (for LIF) or at the N24 time-point (for all other pathway-profiles) (Figure 6a, Supplementary Table 4). Surprisingly, we detected mis-regulated LIF target genes in several KO ESC lines cultured in $2 \mathrm{i}$ in the absence of LIF (Figure 6a). Activation of such a 'LIF-like'-profile in $2 \mathrm{i}$ was closely correlated with the extent of differentiation delay observed at N24 (Supplementary Figure 6b). Notably, presence of LIF together with $2 \mathrm{i}$ before induction of differentiation slows down naïve state exit (Supplementary Figure 6c) 4, 16. Ptpn11, Zfp281, Tsc2 and Trim71 KO ESCs showed the greatest similarity to the LIF profile in $2 \mathrm{i}$; in contrast, Tcf7/1 KO ESCs, despite showing a pronounced differentiation defect, lack the 'LIF footprint' (Supplementary Figure 6b). Addition of a JAK inhibitor to several KOs showing a LIF-like profile resulted in no or only minor amelioration of the differentiation defect. This suggests that these KOs do not directly activate Jak/Stat signalling, but that a LIF-like expression profile reflects a consolidated naïve network that is resilient to dismantling. 
As expected, Raf1, Fgfr2, Mapk1 and Etv5 KO ESCs showed an Fgf-signalling footprint at N24.

Mbd3, Mta3, Nsd1 and Arid5b KOs showed Fgf/ERK target deregulation similar to reference KOs, indicating an involvement of chromatin regulators in ERK target-gene control (Figure 6a). been associated with an exit from naïve pluripotency phenotype ${ }^{45}$. Many KOs (including Pten) downstream effectors) is a common node to gate the exit from naïve pluripotency. Tsc2 depletion resulted in partial activation of a LIF footprint, suggesting that a component of the LIF response is mediated through Akt ${ }^{39,46}$. Rbpj and Tcf7/1 KOs did not show a correlation with each other or with Tsc2 KO, consistent with independent mechanisms ${ }^{21}$. The betacatenin destruction complex member $A p c$ and the previously discussed Jmjd1c showed the expected similarities with the $T c f 7 / 1$ profile. Interestingly, several KOs with strong phenotypes showed footprints similar to $T c f 7 / 1 \mathrm{KOs}$ at N24. This is in line with evidence that ?-catenin constitutes or regulates a major differentiation switch during the exit from naïve pluripotency and is influenced by multiple exit KO genes ${ }^{29,47}$. Genes downstream of the Notch pathway were most strongly affected in a group of mutants deficient for mRNA homeostasis (Smg5 and Smg6) and chromatin regulators, including Jarid2, L3mbt/3 and Mbd3. The latter suggests an interaction between the Notch pathway $(R b p j)$ with the Polycomb and Nurd complexes to modulate network rewiring during the exit from naïve pluripotency. Cooperativity between Rbpj and the Polycomb-associated protein L3mbtl3 has been reported in Drosophila and C. elegans ${ }^{48}$.

We asked to what extent the alteration of any of the five signalling pathways was predictive for the strength of the differentiation defect. We found that whereas aberrant Fgf/ERK or 
mTORC1 activity were unreliable predictors, transcriptional similarity to Rbpj or Tcf7l1 KOs at

N24 or deregulation of LIF target genes in $2 \mathrm{i}$ correlated with differentiation delay

388

389

390

391

392

393

394

395

396

397

398

399

400

401

402

403

404

405

406

407

408

(Supplementary Figure 6d). These data indicate that many KOs with a delay phenotype have altered activity of at least one of the five key signalling pathways. Strikingly, however, there was no single signalling pathway perturbed downstream of all KOs. We observed that KOs showing multiple pathway footprints are rare, but most KOs showing a delayed naïve marker gene downregulation at N24 deregulate at least one specific pathway. Trp53, Ncor1 and Usp9x constitute exceptions, which show appreciable differentiation defects without deregulating of any of the tested signalling cascades. We conclude that signalling pathways are harnessed in parallel and that most KO genes contribute directly or indirectly to at least one pathway activity that promotes the exit from naïve pluripotency.

\section{Identification of gene-networks downstream of multiple KO genes}

To explore common regulatory targets of multiple KO-factors, we classified deregulated genes into two groups: (i), deregulated by a $\mathrm{KO}$ in both $2 \mathrm{i}$ and at $\mathrm{N} 24$, termed 'constitutive $\mathrm{KO}$ response'; (ii) not, or only weakly, deregulated by a $\mathrm{KO}$ in $2 \mathrm{i}$, but significantly deregulated at N24, termed 'N24 induced KO response'. Genes in both groups are affected by knockouts, but genes in the second group deviate from wild-type expression only under conditions of differentiation. We identified all genes that showed either type of behaviour in at least one KO (Supplementary Figure 6e; Supplementary Table 5). Genes deregulated exclusively in PRC2 core-components (Eed or Suz12 KOs) or Csnk1a1 KOs were excluded from further analysis, because the deregulation of thousands of genes in these KOs had a disproportionate impact on the resulting gene-lists. In total, the constitutive and the N24 induced KO response groups contained 1,837 and 720 genes, respectively. Genes belonging to the N24 induced KO 
response group were strongly enriched for factors that are dynamically regulated during normal differentiation, whereas genes of the constitutive response group were not

411 (Supplementary Figure 6f). Thus, naïve marker genes were absent from the constitutive 412 group. Together with NAGs, they were strongly enriched in the N24 induced group, because 413 deregulation of the naïve network only initiates upon $2 i$ withdrawal.

415 To characterize the constitutive KO response genes, we grouped them based on their log-foldchanges between $\mathrm{KO}$ and WT at N24. This resulted in twelve clusters, ranging in size from 20

417 to 597 genes (Supplementary Table 5) (Figure 6b). By Gene Ontology (GO) analysis, eight of 418 the twelve clusters showed a significant enrichment of at least one GO term containing more 419 than five genes (Figure 6c, Supplementary Table 5). Many of these GO terms relate to cell fate specification and development. Notably, the constitutive response clusters often shared identical or similar GO terms, suggesting functional convergence. NAGs were underrepresented in constitutive clusters and only contained in clusters 1 (const $\mathrm{c} 1$ ) and 2, for which expression profiles across all KOs correlated with naïve marker expression (Figure 6b). Therefore, deregulation of genes within const $c 1$ and $c 2$ in $2 i$ is proposed to directly translate to differentiation defects observed at N24. Cluster 1 genes were enriched for general differentiation-related factors and response to Bmp signalling. Constitutive cluster 2 contained a significant number of Fgf/ERK pathway defining genes (Supplementary Figure 7e) and was consistently enriched for Fgf/ERK signalling related terms (Supplementary Table 5). Intriguingly, constitutive cluster 10 contained multiple Zscan4 isoforms ${ }^{49}$ and 14 out of 45 genes in this cluster are bound by the co-repressor Trim28, which is involved in silencing of 
and Zfp281 KOs, but strong downregulation in Rbpj and Jarid2 KOs. Overall, cluster 10

regulation showed no strict correlation with a differentiation delay phenotype.

Analysis of the N24 induced response genes yielded 12 clusters ranging from 8 to 103 genes

(Figure 6d and Supplementary Table 5). All naïve marker genes were detected in N24 induced

cluster 1 (N24i c1), with the notable exception of KIf2. KIf2 was part of N24 induced cluster

10, within which more than half the genes are Tcf7I1 ChIP targets ${ }^{27}$ (Supplementary Figure

7E). This provides further support for the notion that Wnt/Tcf7l1 controls KIf2 expression independently of the rest of the naïve network. Interestingly, twelve percent of genes in N24 induced cluster 10 were screen hit genes, suggesting a major impact of this cluster on the exit from naïve pluripotency (Supplementary Figure 7e). NAGs were distributed in eight out of the twelve N24 induced clusters. In marked contrast to the constitutive KO response, individual N24-induced clusters displayed specific functional enrichments (Figure 6e). Therefore, the

N24 induced response clusters represent distinct molecular pathways and cellular functions that were altered in multiple KOs. N24 induced cluster 7 was significantly regulated in Etv5,

Trim71 and Zfp281 KOs at N24 (Figure 6d). Cluster 7 contains pivotal cell fate switch genes like $\mathrm{Fgf4}, \mathrm{Tfe} 3^{14}$ and $T c f 7 / 1$ itself, but NAGS were underrepresented in this cluster. Notably, Etv5, Trim71 and Zfp281 are the three KO lines that show the strongest exit delay while retaining E4.5 identity at N24 (Supplementary Figure 4d), suggesting that N24 induced cluster genetic modules that are co-regulated by multiple exit factors. 
We asked whether the expression status of these genetic modules correlated with the activity of differentiation regulating pathways. We utilized pathway activity levels and cluster specific expression levels across all KOs to build a model predicting the impact of pathway activity on cluster-gene expression (Figures 7A and S7A to F). Adding further evidence to the pathway to cluster relationships, we found that in most cases activity changes of the regulatory pathways preceded the expression changes of their putative target clusters over the $2 \mathrm{~h}$ differentiation

461 time course. The Notch and mTor pathways showed high connectivity to N24-induced clusters

462 (8 and 6 out of 12 clusters, respectively), whereas Wnt/? ?catenin signalling was specifically 463 correlated with clusters containing high levels of NAGs (N24i c1 and c10) and the only constitutive cluster containing an appreciable number of NAGs (const c1) (Figure 7A).

465 Together, this shows that at least 24 distinct transcription modules can be defined, whose expression is deregulated upon exit gene depletion and which are largely under the control of five key signalling pathways gating differentiation. 


\section{Discussion}

469 Ordered progression through pluripotency requires shutdown of the naïve TF-network and

470 concomitant large-scale rewiring to establish the formative GRN 2, 3, 19 . Following Harald

471 Weintraub's pioneering work with muscle differentiation and Thomas Graf's work on

472 haematopoiesis, the ability of certain transcription factors to change cell identity without a

473 developmental context gained central prominence with somatic cell reprogramming to

474 pluripotency ${ }^{53-55}$. However, most of these studies involved forced expression of selected

475 transcription factors to achieve synthetic cell state transitions in vitro. Here we utilized the

476 remarkable properties of ESCs to recapitulate the pivotal embryonic transition from naïve to

477 formative pluripotency. Notably, we show that this developmental cell state transition is not

478 based on the action of one or two master transcription factors. Although crucial for defining

479 lineage trajectories, transcription factors do not initiate cell fate decisions. Instead, the robust

480 stability of naïve pluripotent stem cells is sustained by multiple crosstalking components and

481 is dismantled by at least four diverse signalling inputs.

482

483 Our dissection of an authentic cell state transition indicates that the exit from naïve 484 pluripotency is largely guided along a trajectory constrained by signalling pathways and 485 funnelled through a handful of genetic modules. These adaptations of cellular networks establish the opportunity for TFs to initiate and consolidate cell identity. In the specific case

487 of the exit from naïve pluripotency, expression of Oct4 and Sox2, which are key TFs for both the naïve and formative states, provide cornerstones for both the naïve and formative regulatory networks to ensure lineage fidelity during differentiation (Figure 7B). Our data 
of signalling and the chromatin modification machinery precedes the initiation of a novel cell fate.

493

494

Several signalling-cascades have been implicated in ESC self-renewal and naïve pluripotency exit, including pathways controlled by LIF, Akt/mTOR, Wnt, Fgf/ERK and Notch. We derived transcription footprints of these pathways and scrutinized the extent to which they were deregulated by the different KOs to find that virtually all KOs showing a strong differentiation defect also showed deregulation of at least one of these five pathways. Notably, however, we observed segregation in terms of pathway deregulation. Thus, KOs that induce e.g. a Tcf7/1-

like profile were less likely to show a signature of another pathway. This suggests that ESC of separable genetic networks. The observation that single gene depletion is not sufficient to prevent exit from the naïve state is consistent with this interpretation ${ }^{21}$. Our analyses exposed discrete pathway and GRN features that mediate timely and robust mammalian cell state transitions. We found that 12 genetic sub-networks (clusters) with non-overlapping cellular functions emerge during the exit from naïve pluripotency. Interestingly, signalling pathway activity was closely co-regulated with these clusters suggesting a functional linkage. genetic modules under control of, or controlling, key signalling pathway activities.

511 By examining co-regulation with the core naïve network across 146 perturbations (73 KOs in

$5122 \mathrm{i}$ and at N24) we identified the NAGs. NAGs obey strikingly similar expression dynamics in 
naïve pluripotency. Therefore, this cohort of genes constitutes a layer of the pluripotency network that is tightly linked to, and likely acts in conjunction with, the core naïve TF-network in mammals ${ }^{4}$. We suggest that collective modulation of NAG expression will propel naïve cells into formative differentiation and conversely, that collective NAG deregulation will delay proper differentiation. The control of NAG expression appears to be interwoven with the signalling pathways involved in controlling the naïve to formative transition. The N24 induced clusters enriched for NAGs showed high connectivity to all tested pathways. The functional significance of NAGs likely extends beyond self-renewal and naïve identity. Indeed, most NAGs are not transcription factors. The growth factor Pdgfa is a case in point. Pdgfa is the most strongly associated NAG, but has no known activity on ESCs, which do not express the cognate receptor, Pdfgra. Within the ICM, Pdgfa expression is restricted to the naïve epiblast, whereas Pdgfra is present exclusively in the primitive endoderm. This reciprocal expression pattern is consistent with the known paracrine action of Pdgfa to promote primitive endoderm segregation ${ }^{35}$. Thus, conserved linkage of NAGs to the naïve TF network may mediate paracrine communication to other lineages within the blastocyst as well as consolidate naïve epiblast fate. expressed in mouse ESCs and naïve epiblast, it is very lowly expressed in primate blastocysts, and also in the porcine pluripotent compartment ${ }^{56}$. KIf2 may therefore be a rodent specific addition to the naïve TF-network. A further significant species difference is the very low expression of TCF7L1 in primate naïve cells, which underlies the differential responsiveness

538 of mouse and human naïve cells to GSK3 or Wnt pathway inhibition ${ }^{34,57}$. Klf2 is a genomic 
target of Tcf7l1 ${ }^{27}$. Interestingly, KIf2 and Tcf7l1 are found in the same N24 induced geneexpression cluster (cluster 10), suggesting coregulation of both components of this mouse specific naïve pluripotency feedback loop. For most KOs the degree of failure in naïve TF downregulation and the neutralisation of global differentiation-related transcription changes are comparable. However, the relatively weak Trim71 phenotype despite specific must reach a certain threshold to stably maintain naïve pluripotency, consistent with negative feedback constraints within the network $4,5,58$.

The remarkable advantages of ESCs as an experimental venue include the utilisation of welldefined culture conditions to recapitulate key features of in utero developmental progression hours in vitro mimics the peri-implantation transition from naïve to formative epiblast in utero. These data provide rich resources for further studies. In particular, many strong differentiation delay KO transcriptome profiles retained transcriptome resemblance to the pre-implantation epiblast. This relationship held true in $2 \mathrm{i}$ and at N24 and indicates that the $\mathrm{KO}$ of these genes perturb the operative in vivo cell state transition machinery. In general, we surmise that stalling the activity of the exit machinery is critical to in vitro capture and selfrenewal of naïve stem cells, consistent with empirical requirements for signal inhibition in

558 both mouse and human ${ }^{7,57}$. Interestingly, deficiency for some differentiation drivers increases similarity of $2 \mathrm{i}$ profiles to the in vivo pre-implantation epiblast. Therefore, we

560 propose that some of the mechanisms driving exit from naïve pluripotency contribute to the

561 lack of complete identity between in vivo E4.5 epiblast and ES cells in 2i. This is in line with 562 ESCs representing an engineered interruption in developmental progression and suggests 
563 that state-of-the-art self-renewal culture conditions may not yet capture the naïve state

564 perfectly ${ }^{1}$.

565

566 Our analyses provide a comprehensive inventory of genetic factors and regulatory networks

567 governing the major cell fate transition from pre- to post-implantation epiblast (Figure 7B).

568 We present evidence that mammalian development, employs similar regulatory networks

569 operating with similar mechanisms ${ }^{61,62}$. These programmed cell state transitions in

570 development do not rely upon instructions delivered by one or two master transcription

571 factors. Rather, a cloud of activity, involving multiple co-ordinated inputs, serves to destabilise

572 an existing, multiply stabilised GRN. Thereby a consolidated cell state can be rapidly

573 dismantled by a combination of signals, triggering transition to the next developmental

574 waystation. 
575

576

577

578

579

580

581

582

583

584

585

586

587

588

589

590

591

592

\section{Acknowledgements}

We thank Johanna Stranner, Thomas Sauer and Andy Riddell for help with flow cytometry. Meng Li and Kosuke Yusa for sharing RC9 cells, Andreas Dahl for NGS-sequencing support and Christa Bücker for critical comments on the manuscript. ML is funded by a WWTF-VRG grant (VRG14-006). This study was supported by an FWF/DFG DACH grant to AB and ML (FWF grant number: I 3786; DGF grant number 398882498). AB and MG were supported by the BMBF (Sybacol). RS received support by the Cologne Graduate School of Ageing Research. Wellcome and the Medical Research Council provide core support for the Wellcome-MRC Cambridge Stem Cell Institute. AS is an MRC Professor. This project was initiated within the EU FP7 integrated project SyBoSS (Grant agreement: 242129) co-ordinated by AFS and AB including partners $\mathrm{ML}$ and $\mathrm{AS}$.

\section{Author contributions:}

$A B, M L, A S$ and $A F S$ conceptualized the study. $A B, M L$ designed the study. $A L, M L, A B, R S, M G$ and FTT designed experiments. AL, MH, JR, PvdL, HFT, LS and MS carried out wet-lab experiments. RS, MG, GGS, FTT and AL performed bioinformatic analyses. ML, AB, AS, AFS wrote the paper with input from AL, RS, MG, MH, FTT and GG. Funding was acquired by ML, $A B, A F S$ and $A S$. 
bioRxiv preprint doi: https://doi.org/10.1101/2020.03.23.000109; this version posted July 2, 2020. The copyright holder for this preprint (which was not certified by peer review) is the author/funder. All rights reserved. No reuse allowed without permission.

a

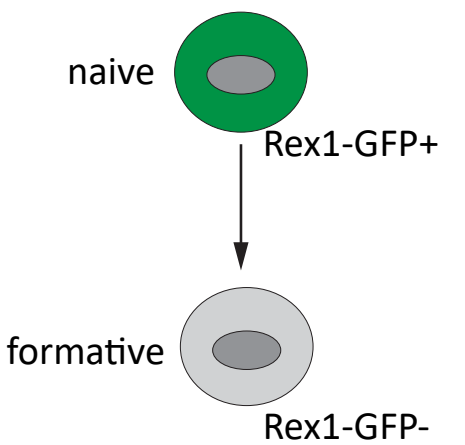

C

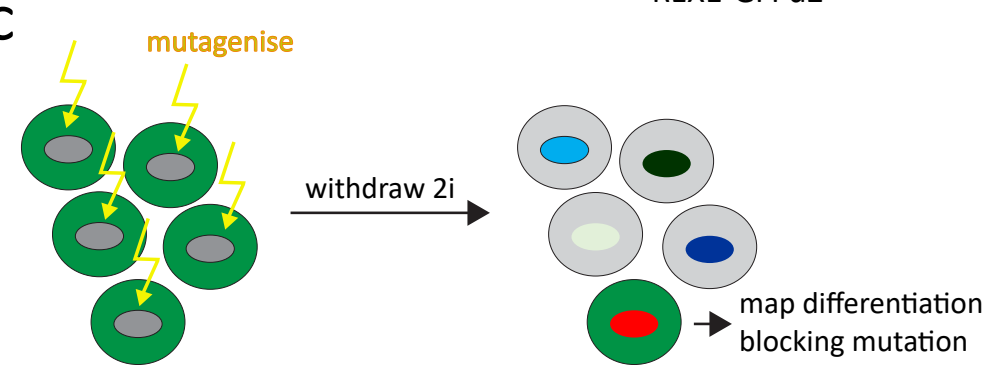

N24

e

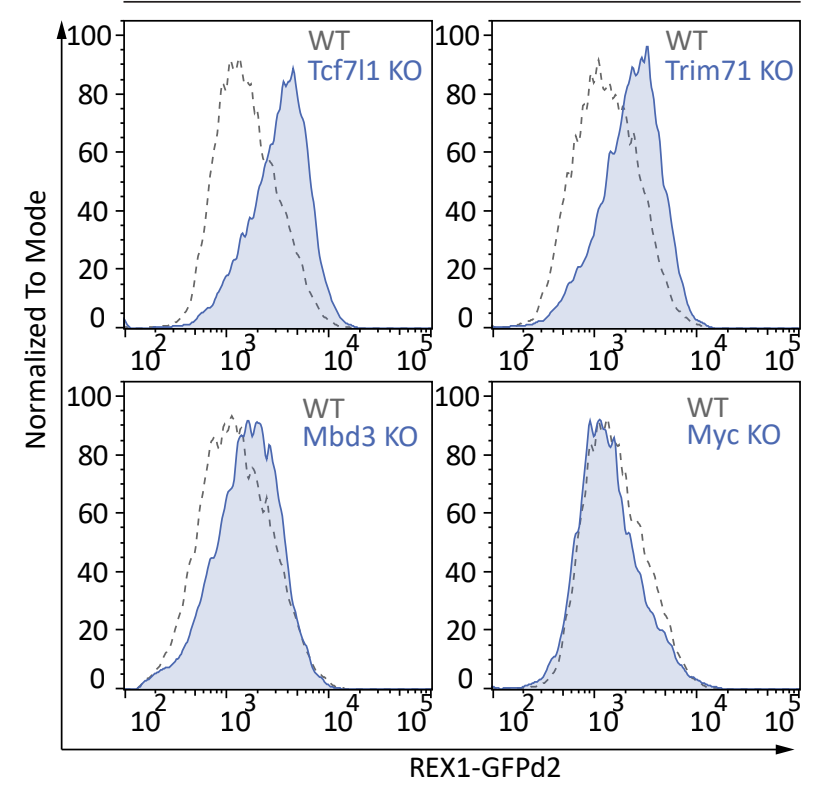

g
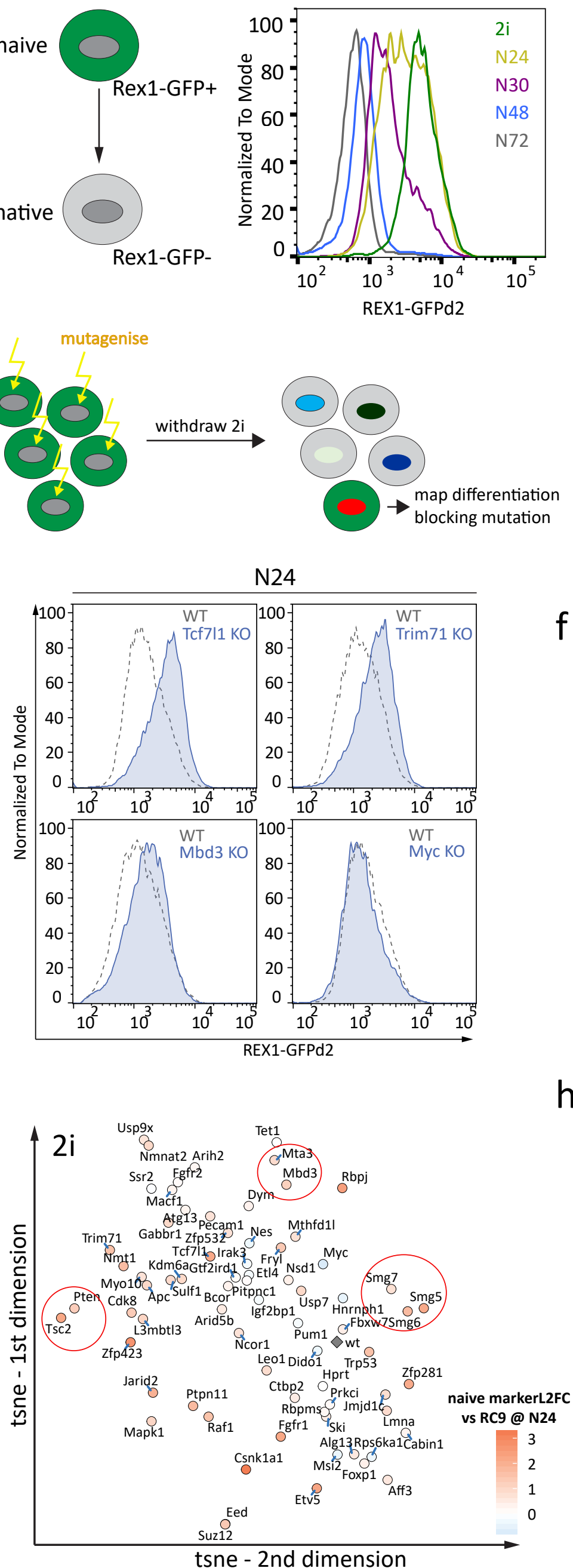

h d

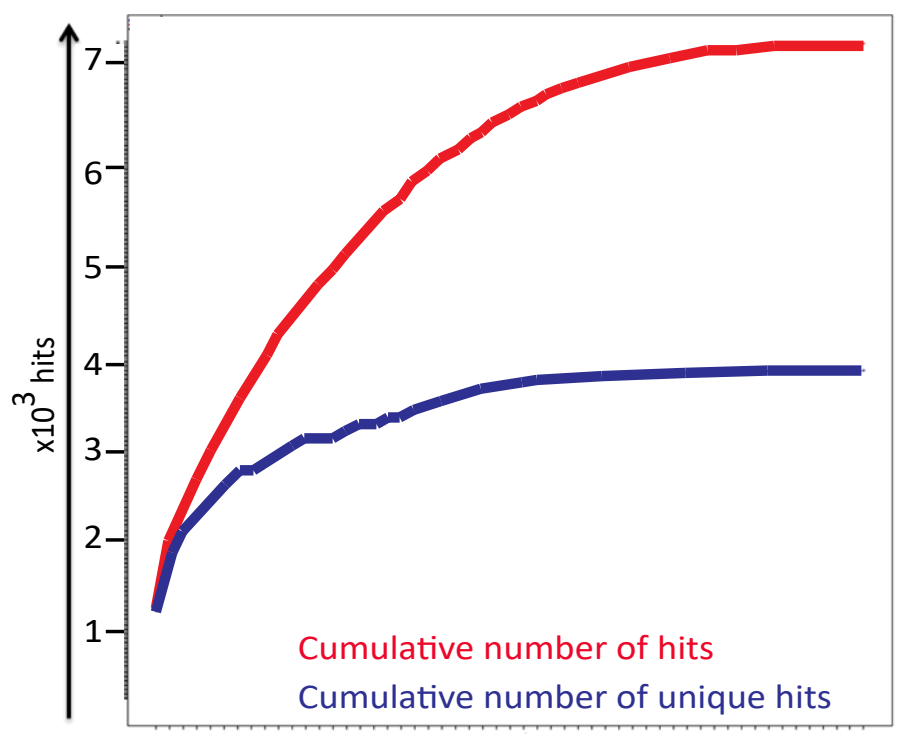

35 screens
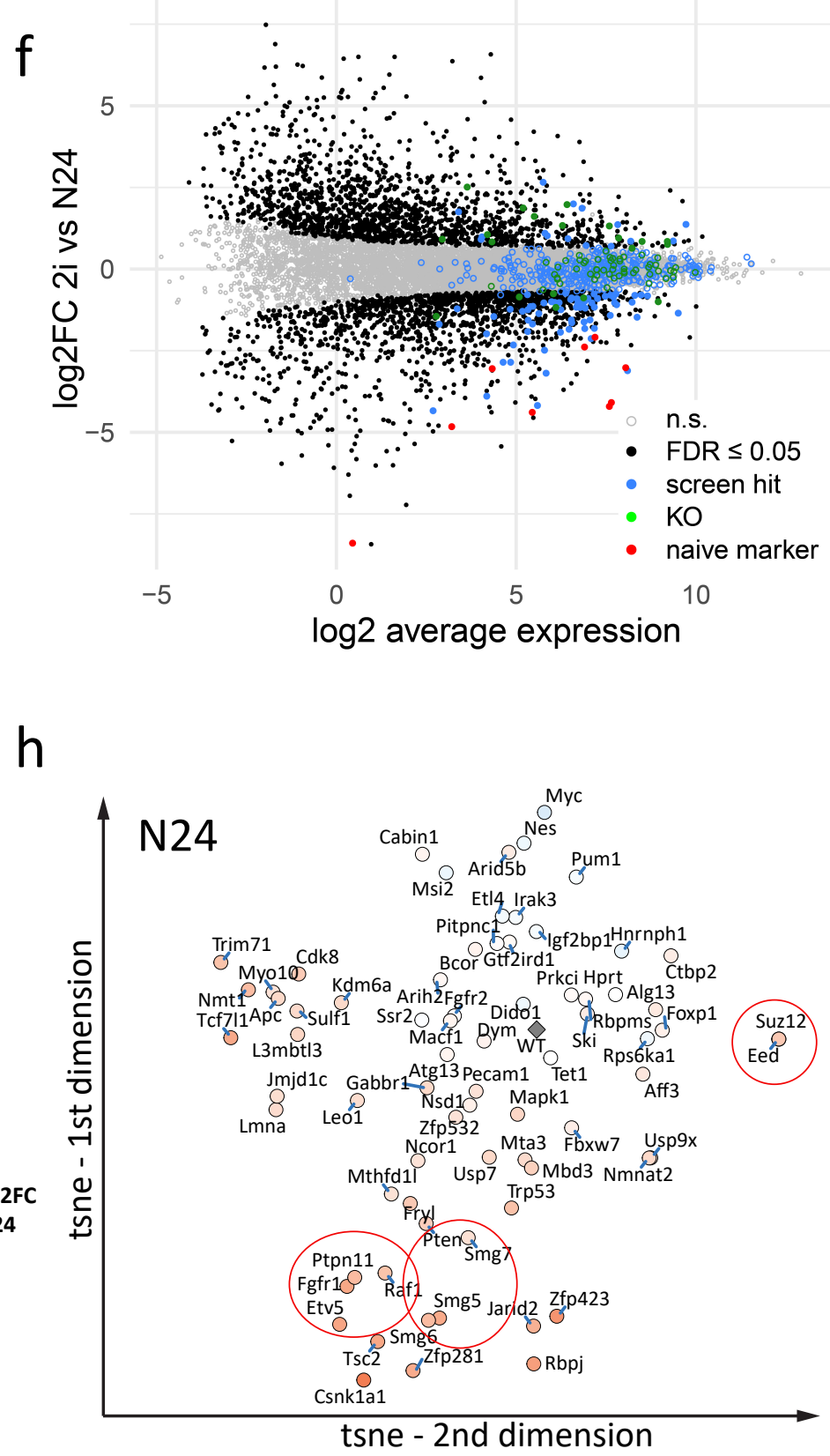
bioRxiv preprint doi: https://doi.org/10.1101/2020.03.23.000109; this version posted July 2, 2020. The copyright holder for this preprint

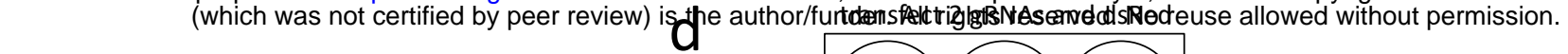

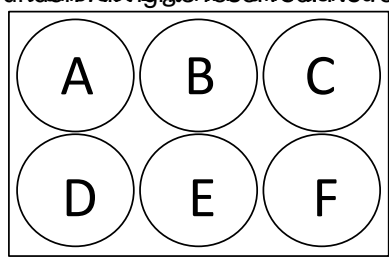

times hit in integration

\begin{tabular}{lcrr} 
Gene & 35 screens & sites & \multicolumn{1}{c}{$\mathrm{fdr}$} \\
\hline Tcf7l1 & 34 & 166 & $1 \mathrm{E}-232$ \\
Fgfr2 & 25 & 78 & $6.1 \mathrm{E}-21$ \\
Alg13 & 20 & 35 & $9.3 \mathrm{E}-39$ \\
Ctbp2 & 17 & 43 & $5.6 \mathrm{E}-45$ \\
Arfip1 & 17 & 29 & $4.5 \mathrm{E}-34$ \\
Jarid2 & 16 & 35 & $7 \mathrm{E}-31$ \\
Rbpj & 16 & 25 & $7 \mathrm{E}-19$ \\
Nsd1 & 14 & 29 & $1.1 \mathrm{E}-26$ \\
Arid5b & 14 & 17 & $1.9 \mathrm{E}-11$ \\
Fbxw7 & 13 & 25 & $1.9 \mathrm{E}-16$ \\
Msi2 & 13 & 24 & $1.2 \mathrm{E}-12$ \\
Dym & 13 & 16 & $6.6 \mathrm{E}-07$ \\
Etv5 & 12 & 26 & $8 \mathrm{E}-30$ \\
Mapk1 & 12 & 19 & $1.1 \mathrm{E}-16$ \\
Smg7 & 12 & 19 & $3.5 \mathrm{E}-15$
\end{tabular}

Beta-catenin phosphorylation cascade Regulation of lipid metabolism by PPARalpha SHC-mediated cascade:FGFR1

Regulation of PTEN gene transcription catenin transactivating complex Deactivation of $\mathrm{b}$-catenin transactivating complex
Intrinsic Pathway for Apoptosis
TP53 Regulates Metabolic Genes
Signaling by FGFR2
Regulation of HSF1-mediated heat shock response
Transcriptional activity of SMAD2/3:SMAD4 Deactivation of $\mathrm{b}$-catenin transactivating complex
Intrinsic Pathway for Apoptosis
TP53 Regulates Metabolic Genes
Signaling by FGFR2
Regulation of HSF1-mediated heat shock response
Transcriptional activity of SMAD2/3:SMAD4 Deactivation of $\mathrm{b}$-catenin transactivating complex
Intrinsic Pathway for Apoptosis
TP53 Regulates Metabolic Genes
Signaling by FGFR2
Regulation of HSF1-mediated heat shock response
Transcriptional activity of SMAD2/3:SMAD4 Transcriptional activity of SMAD2/3:SMAD4 PIP3 activates AKT signaling RHO GTPase Effectors

TCF dependent signaling in response to WNT Transcriptional Regulation by TP53 Metabolism of RNA Cellular responses to stress Signaling by Receptor Tyrosine Kinases Cell Cycle, Mitotic

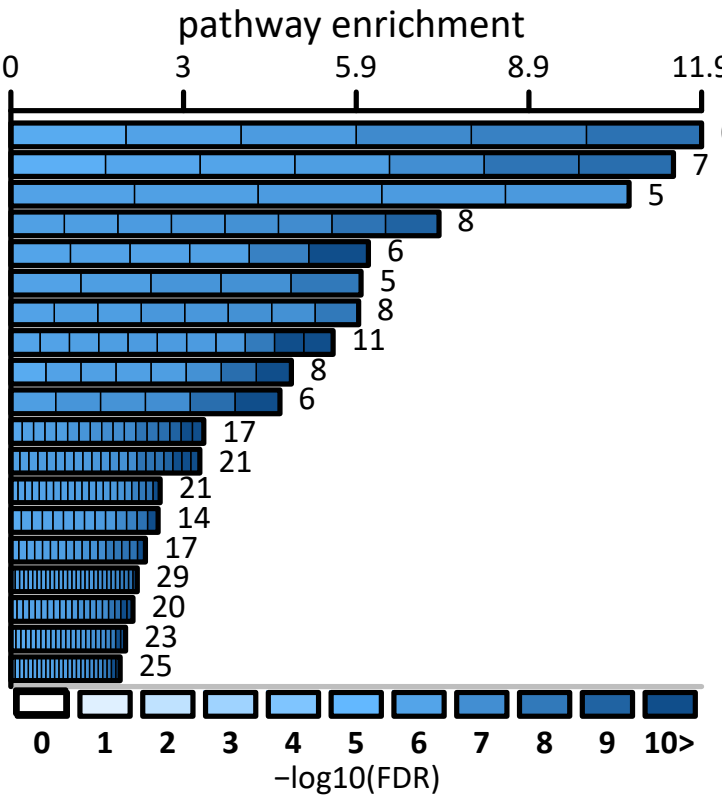

e

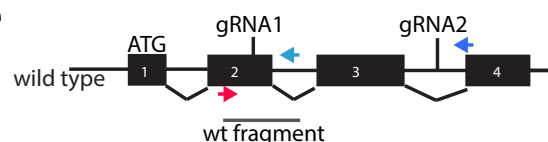

d2
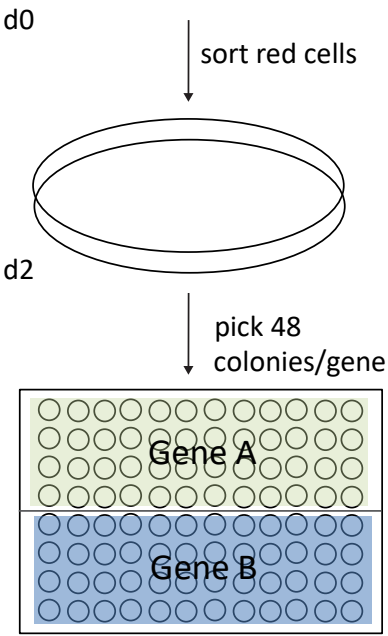

d9

GO enrichment

C

retinoid $X$ receptor binding cerebellum morphogenesis Fgfr signaling pathway histone methyltransferase complex hindbrain development positive regulation of Wnt signaling pathway

stem cell population maintenance regulation of morphogenesis of an epithelium negative regulation of epithelial cell proliferation regulation of DNA replication neural tube closure stem cell differentiation regulation of gene expression, epigenetic skin development lung development response to growth factor stimulus DNA-binding transcription activator activity, epithelial tube morphogenesis cell adhesion molecule binding

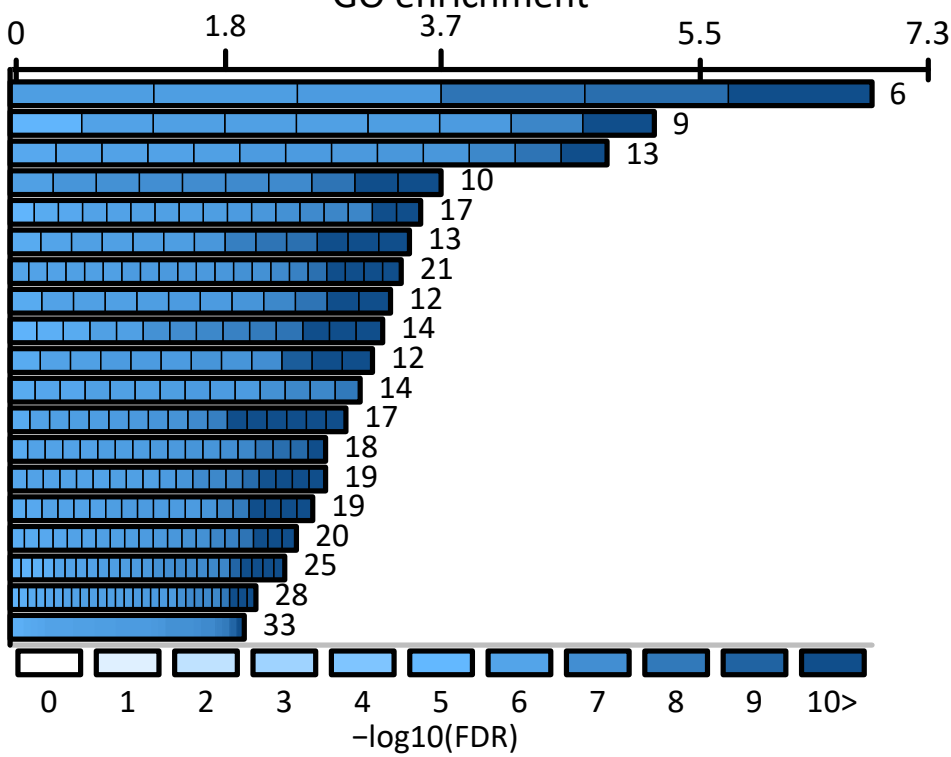

Supplementary Figure 1 
bioRxiv preprint doi: https://doi.org/10.1101/2020.03.23.000109; this version posted July 2, 2020. The copyright holder fordthis preprint (which was not certified by peer review) is the author/funder. All rights reserved. No reuse allowed without pe\&misssigar. "co

f

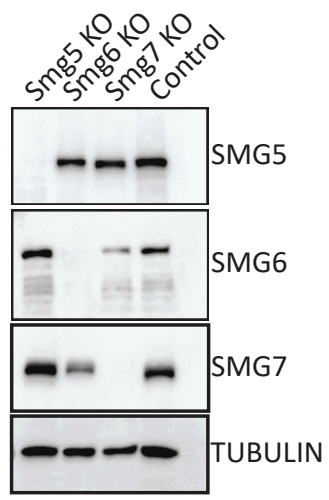

20

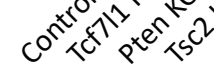
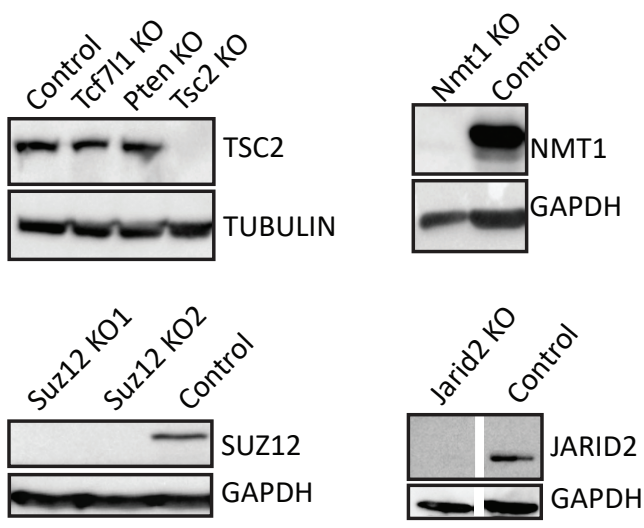
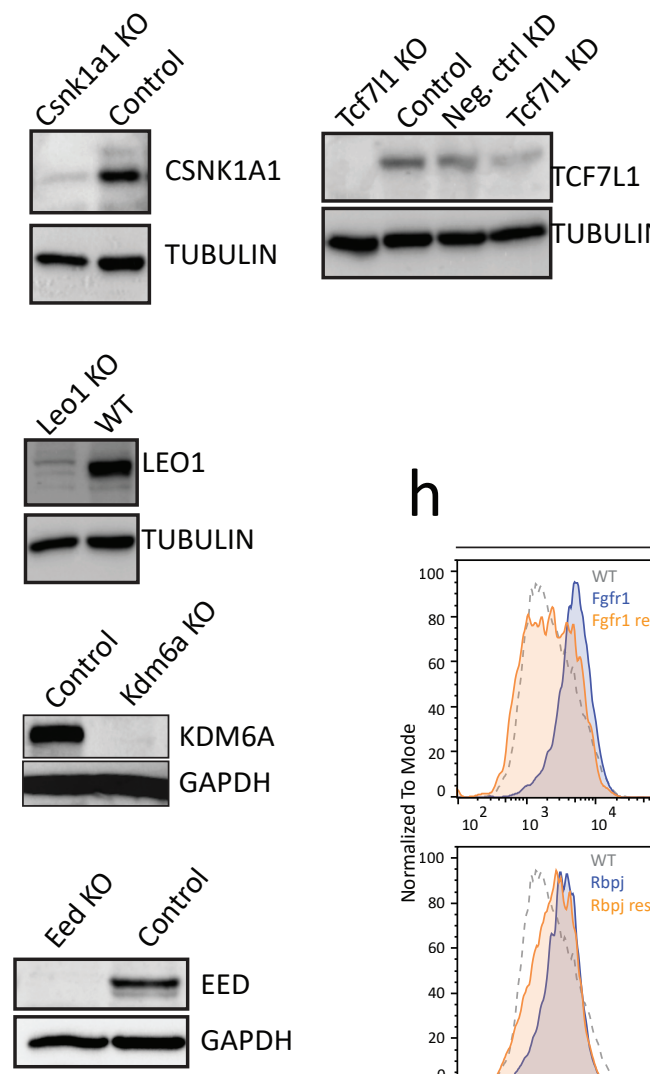

h
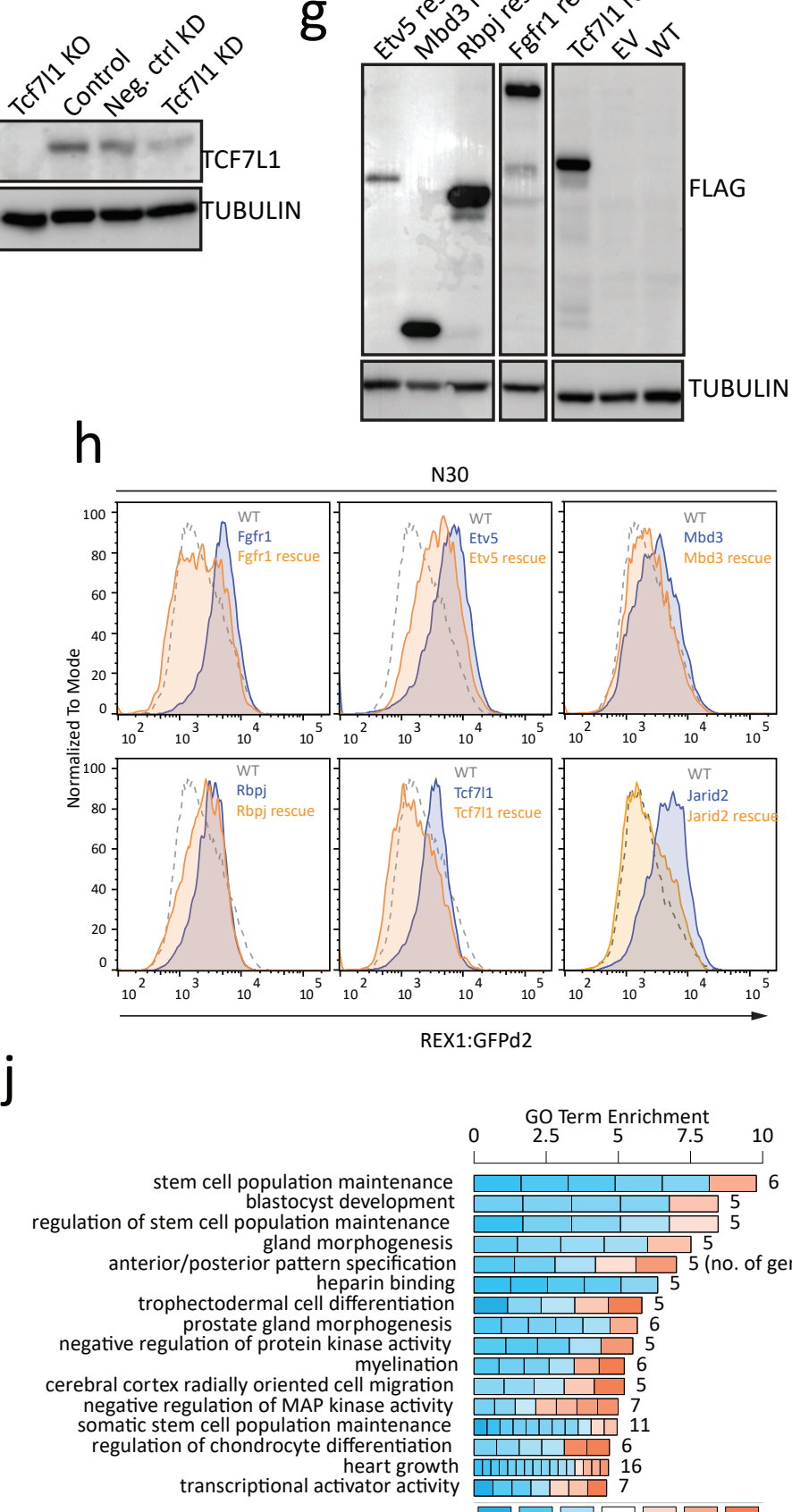

tSNE 2nd dimension

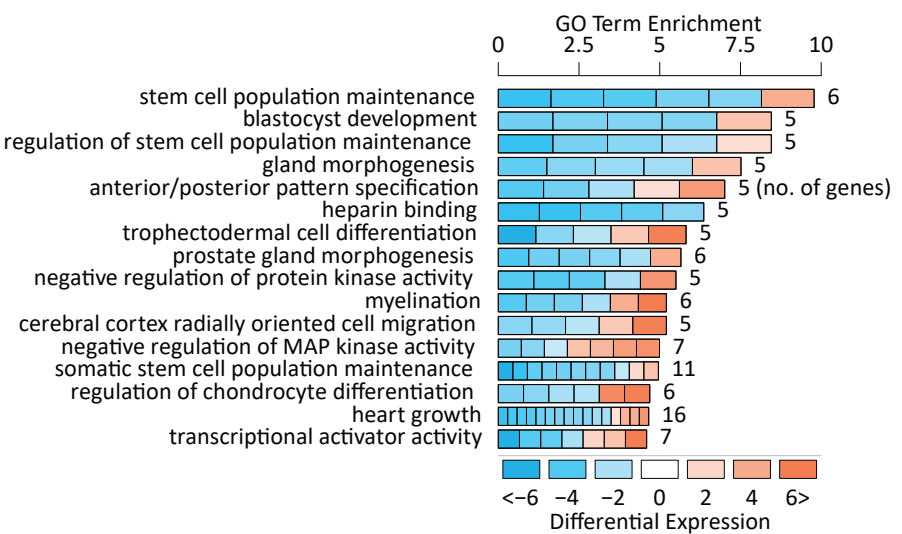

TLS: $y=-0.06+0.18 x$

$\mathrm{m}$
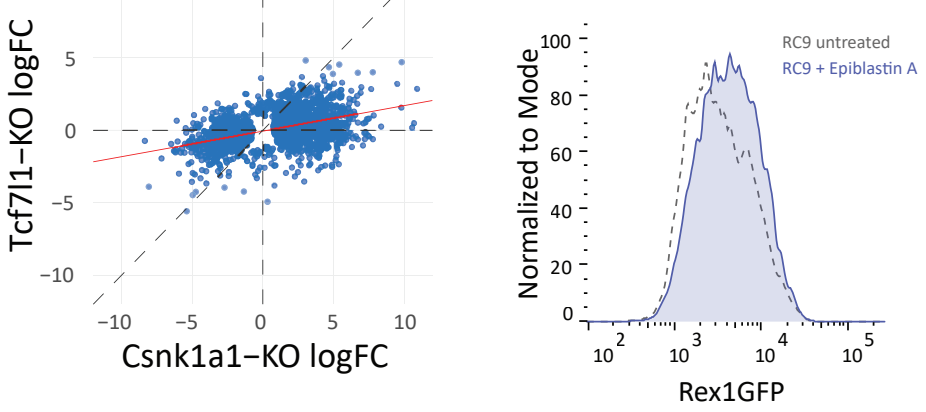

100: early passage

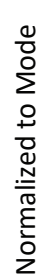

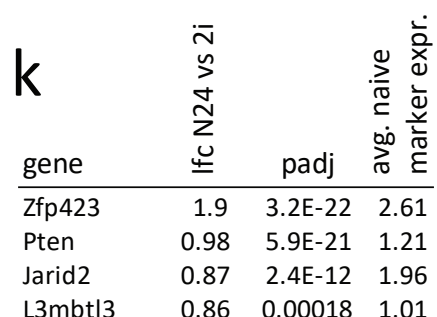

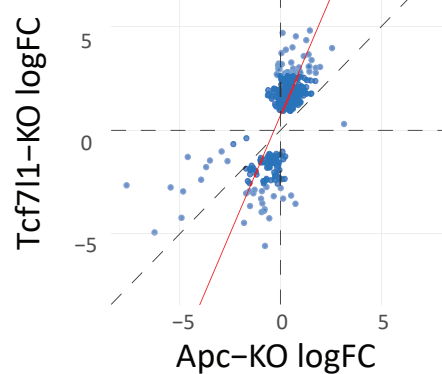

2i WT

N24 Pum1 KO N24 WT

4 passages

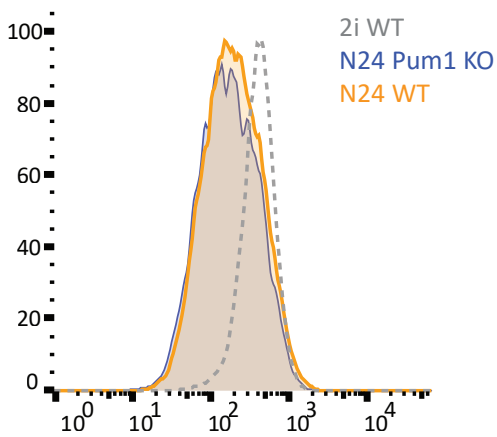

Supplementary Figure 1 
bioRxiv preprint doi: https://doi.org/10.1101/2020.03.23.000109; this version posted July 2, 2020. The copyright holder for this preprint (which was not certified by peer review) is the auther/funder. All rights reserved. No reuse allowed without permission.

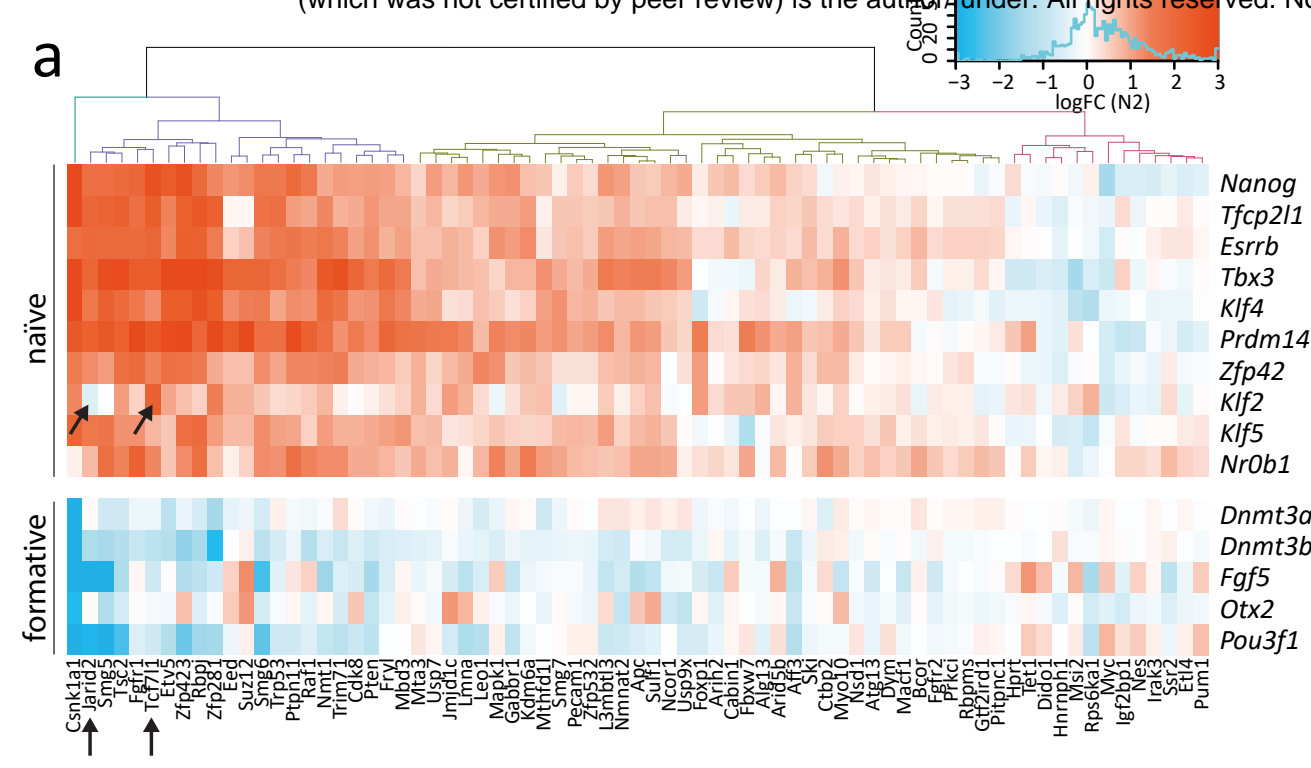

b

C

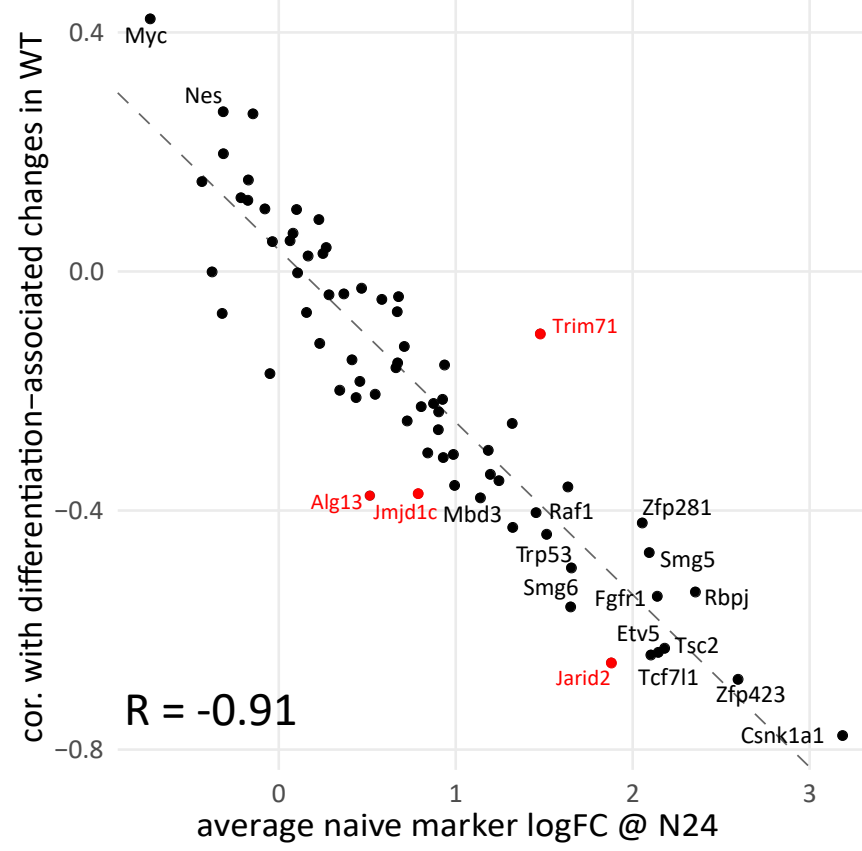

d

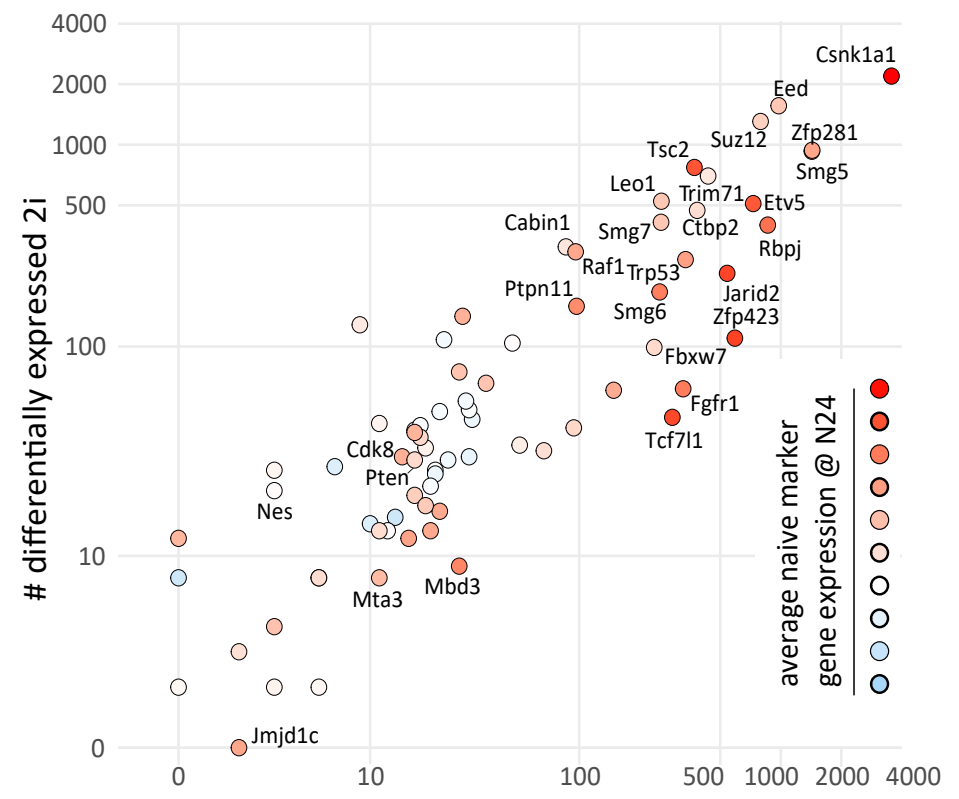
\# differentially expressed N24

e
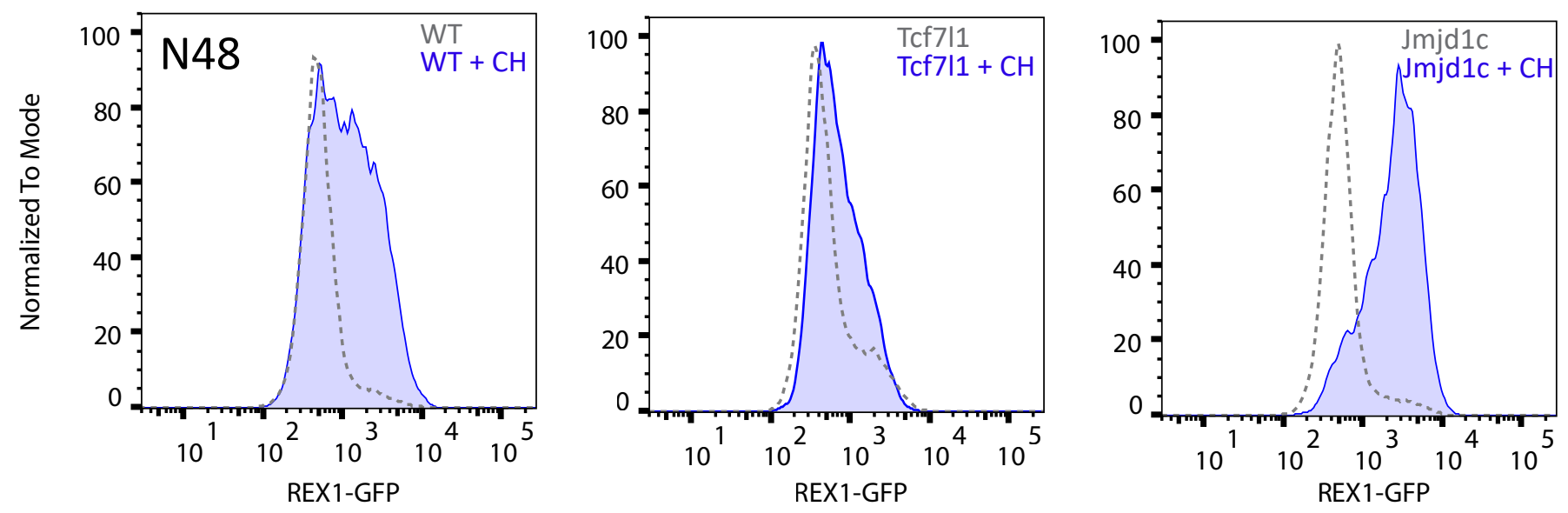

Figure 2 
bioRxiv preprint doi: https://doi.org/10.1101/2020.03.23.000109; this version posted July 2, 2020. The copyright holder for this preprint (which was not certified by peer review) is the author/funder. All rights reserved. No reuse allowed without permission.

a

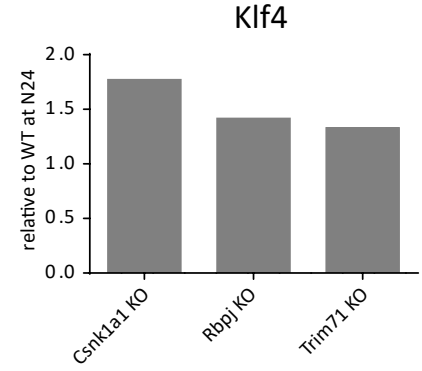

C

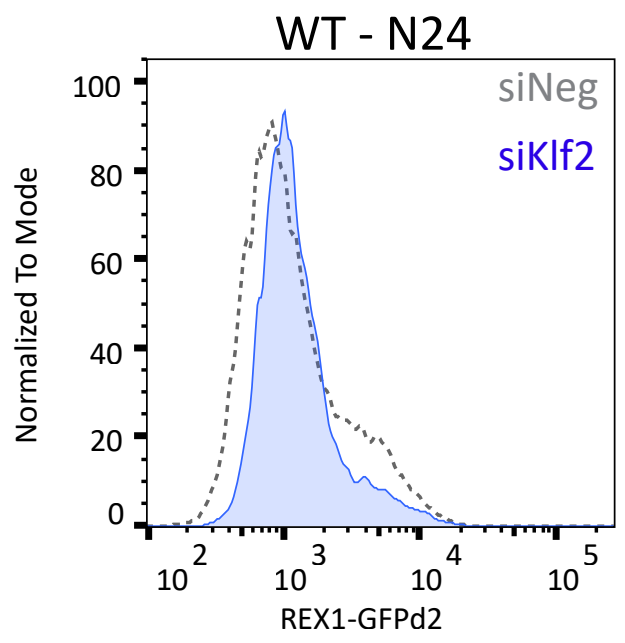

Otx2
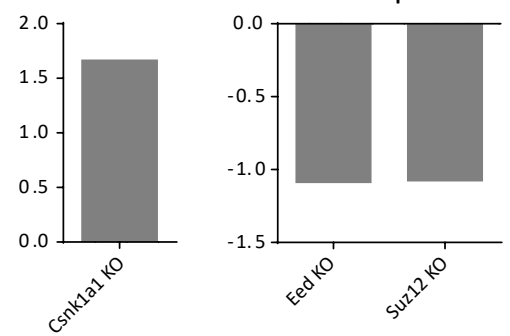

b

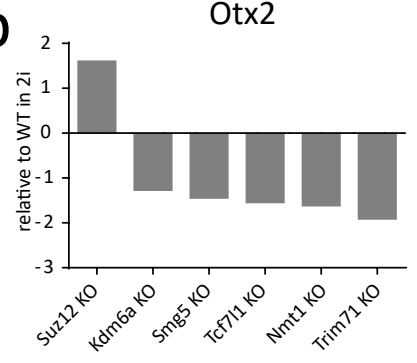

Fgf5

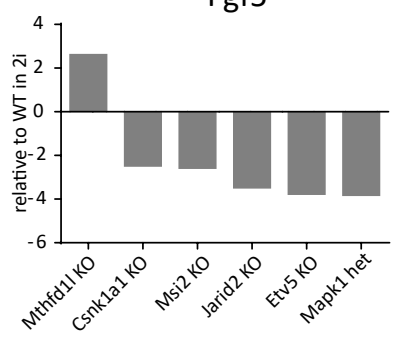

Pou3f1

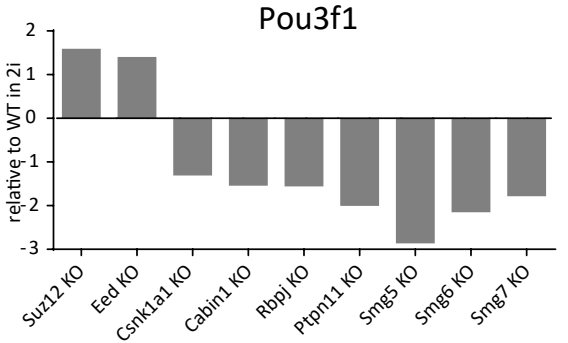

e

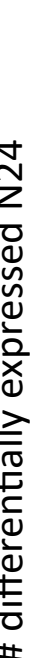

4000

2000

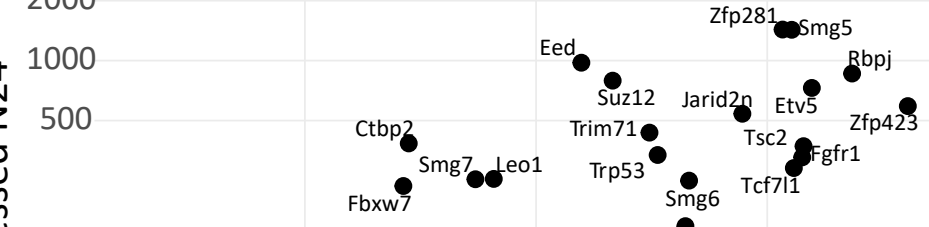

Csnk1à

100

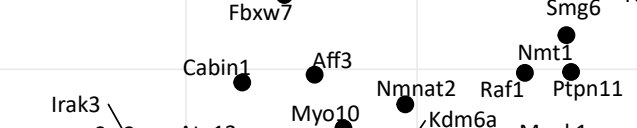

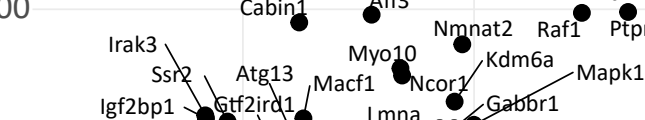

Igf2bp1 Getf2ird1/ Macf1 Lmna

$13 \mathrm{mbtl} 3 \mathrm{Mbd}$

Pitpnc1 1 Sulf 1 -Dym

10

Nes Fgfr2 Arih2 Esp9x Mta3 Cdk8 Fryl

aHnrnph1

Myc Msi2 $\omega^{\text {Rps6ka1 }}$ Arid5b

Hprt

0

Pum1

Jmjdic Usp7

eAlg13

0

1

2

3

average naive marker logFC @ N24

Supplementary Figure 2 
a

\section{KOESCs@ N24}

compare KO N24 expression to WT RNA-seq time-course

WT: $2 \mathrm{i} \rightarrow 2 \mathrm{~h} \rightarrow 4 \mathrm{~h} \rightarrow 6 \mathrm{~h}$ $\rightarrow 32 \mathrm{~h}$

C

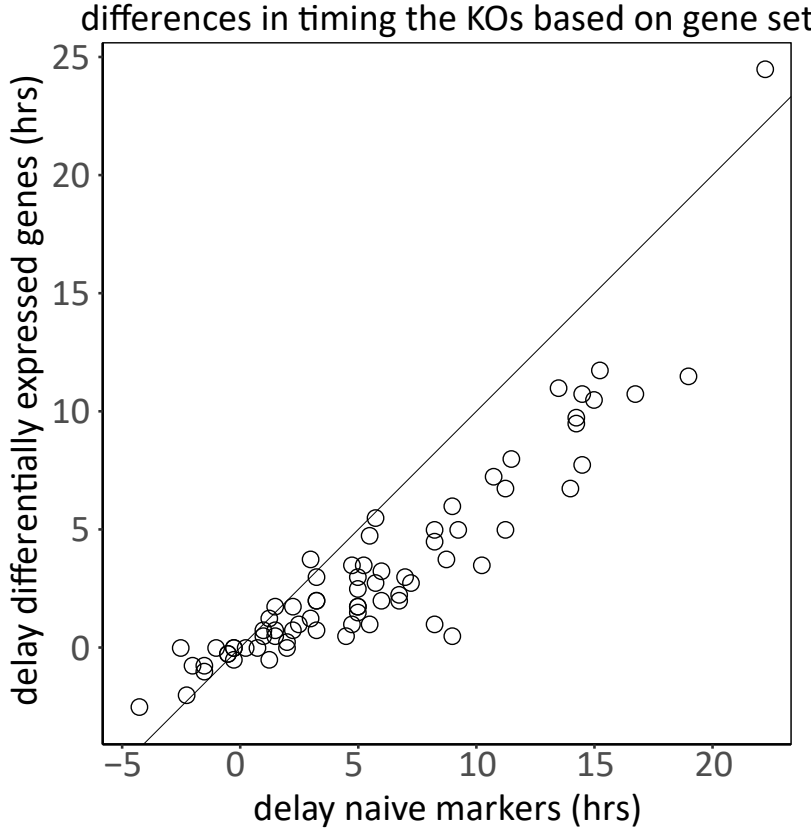

b

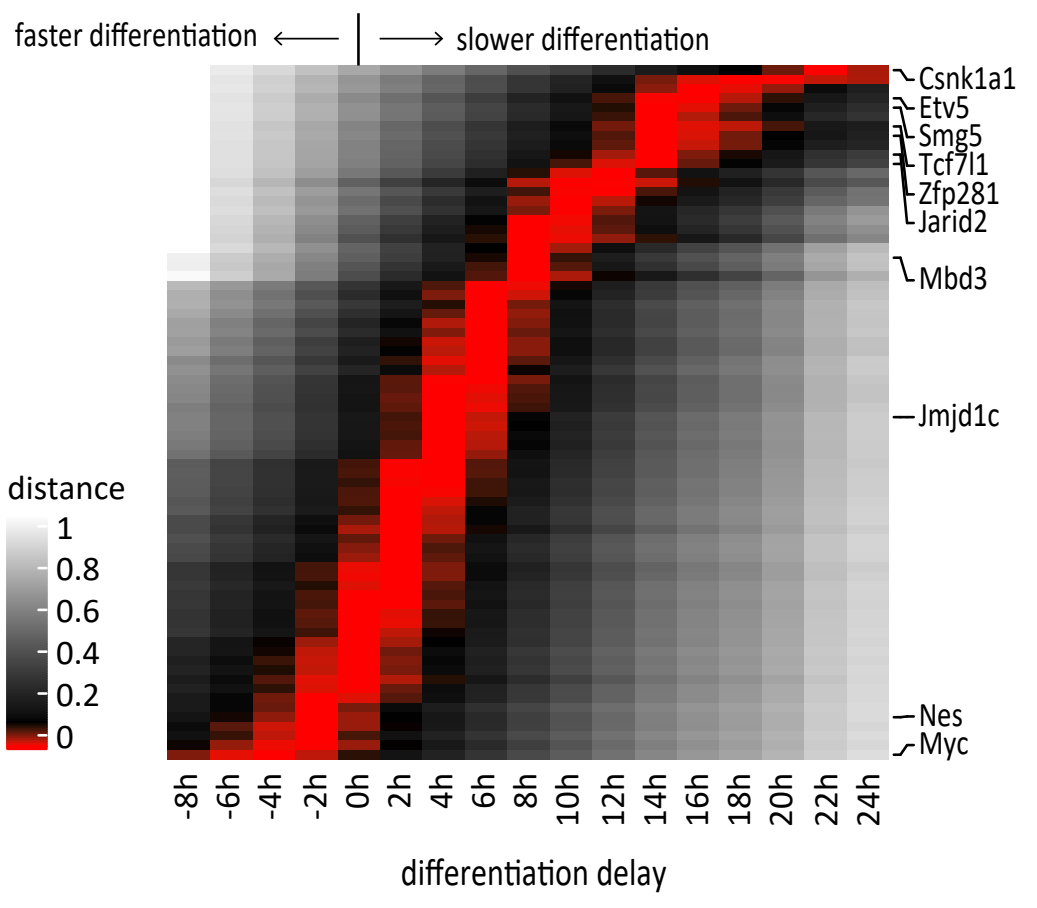


a

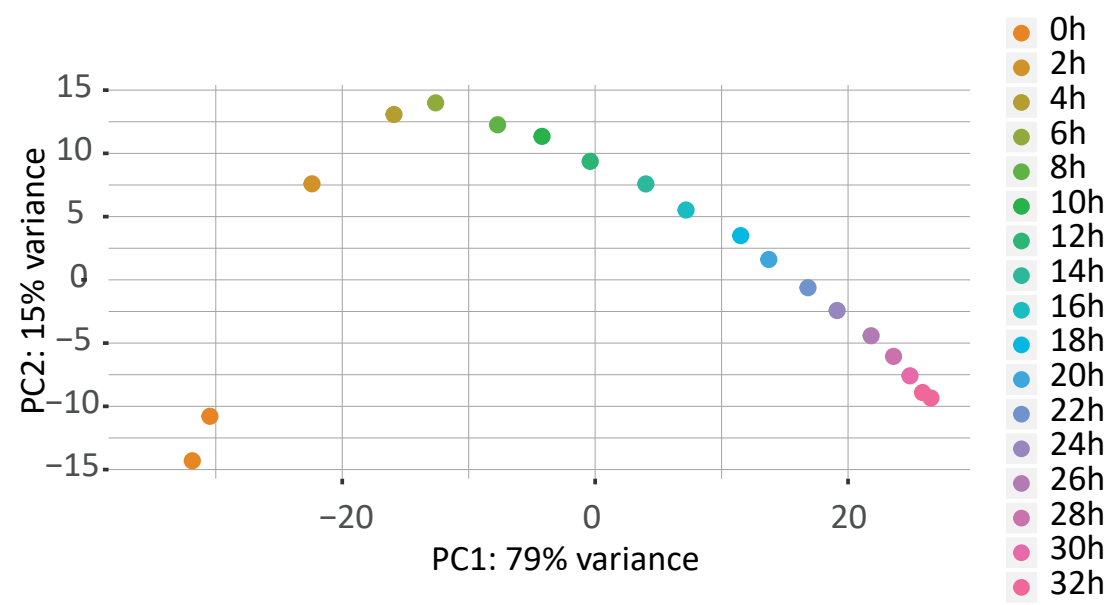

$\mathrm{b}$

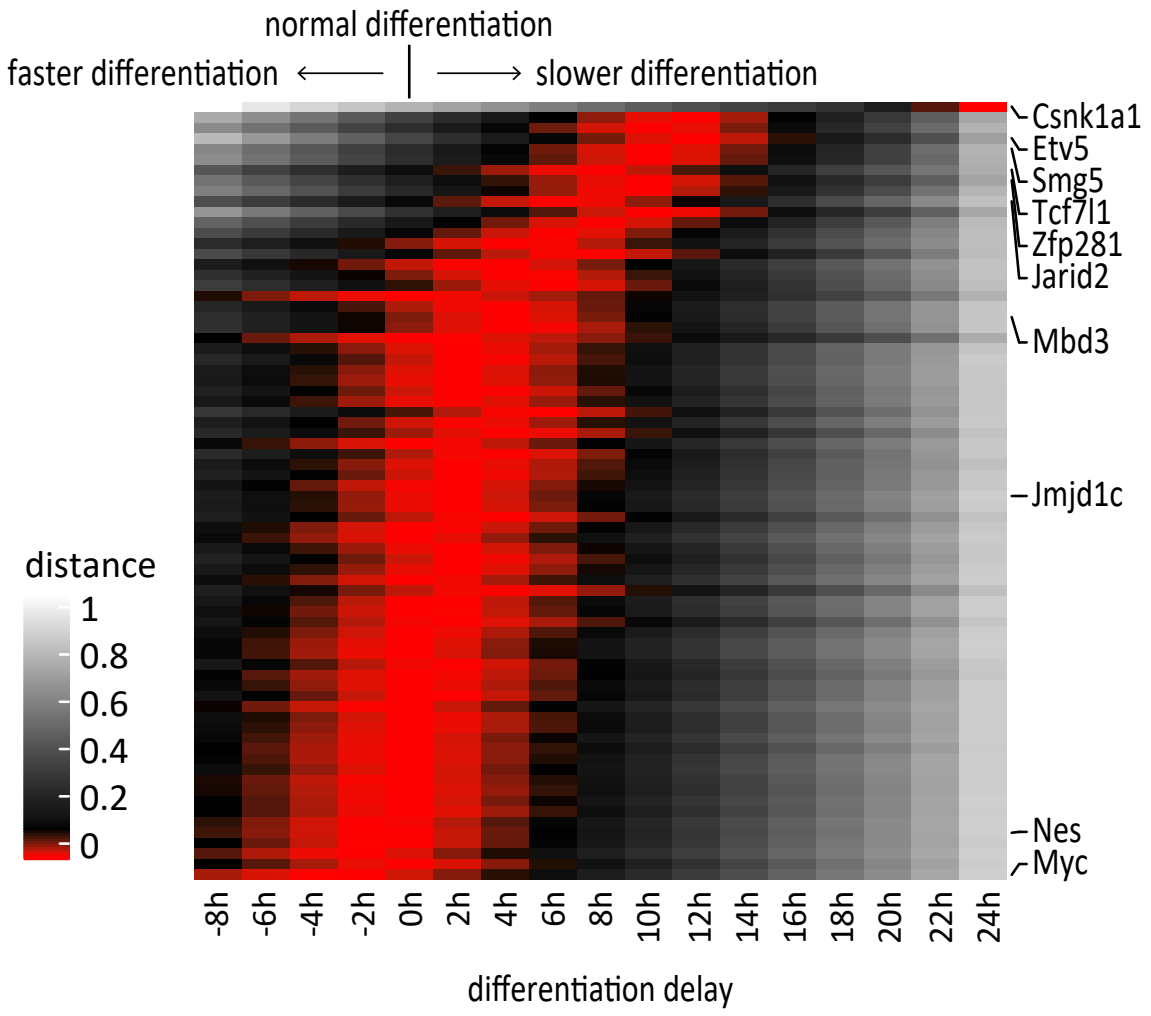

Supplementary Figure 3 
bioRxiv preprint doi: https://doi.org/10.1101/2020.03.23.000109; this version posted July 2, 2020. The copyright holder for this preprint (which was not certified by peer review) is the author/funder. All rights reserved. No reuse allowed without permission.

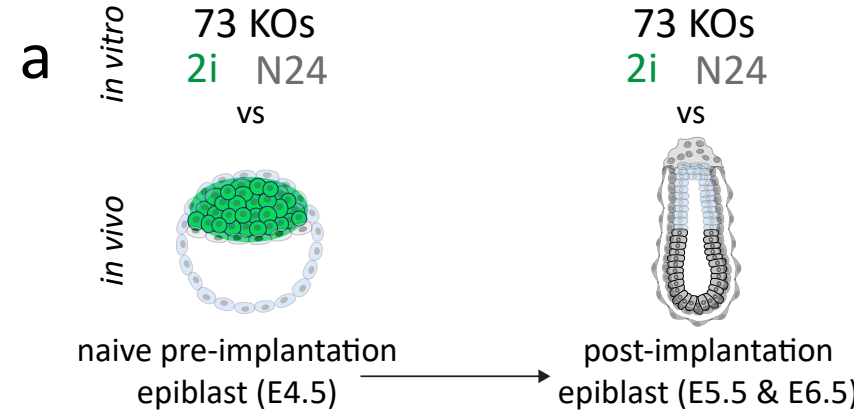

C

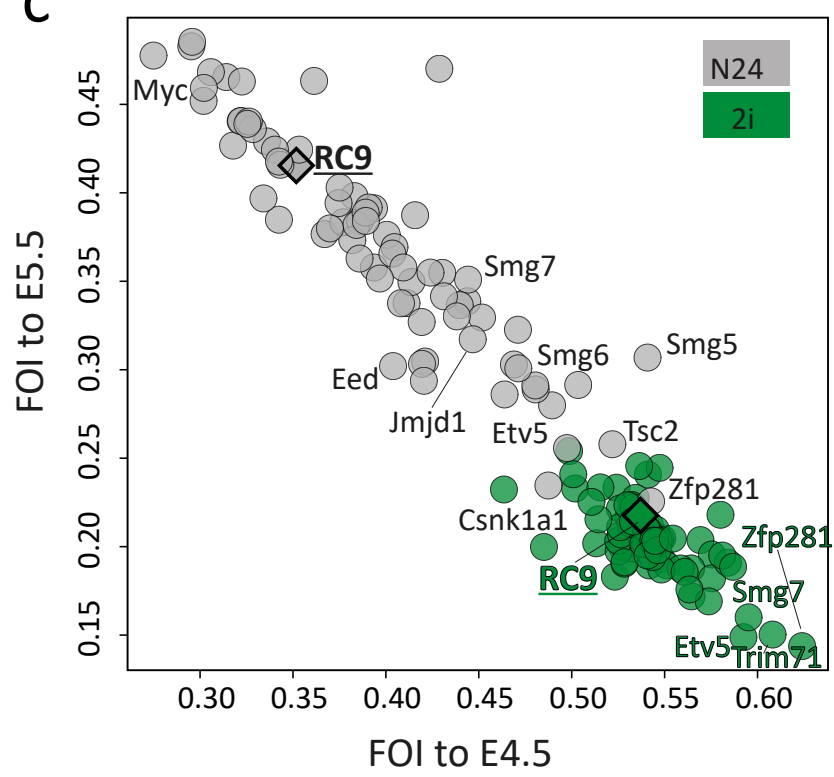

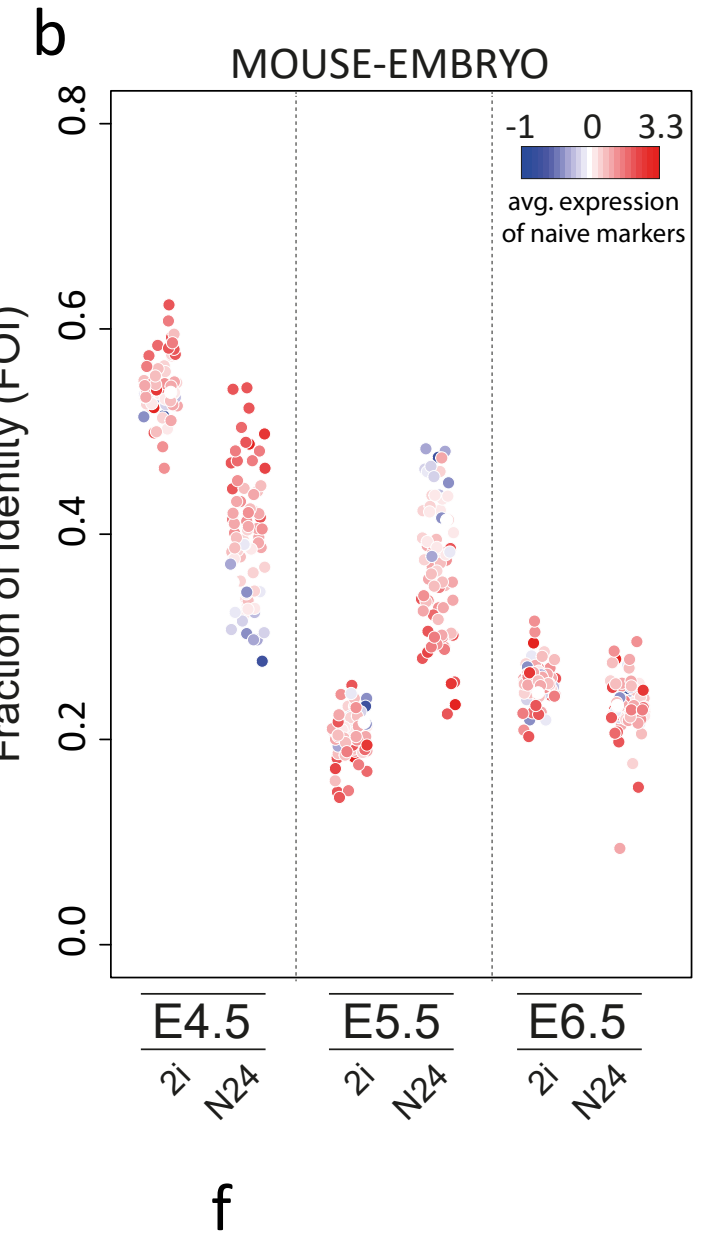

$\mathrm{e}$

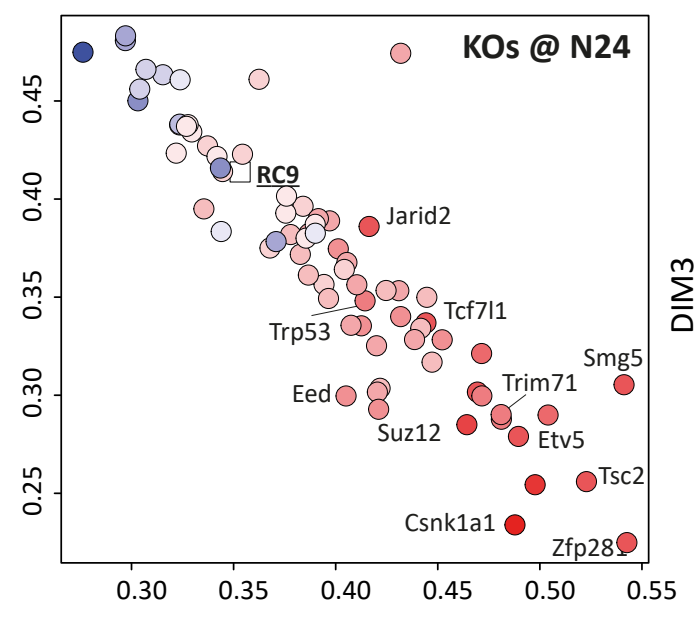

FOI to E4.5
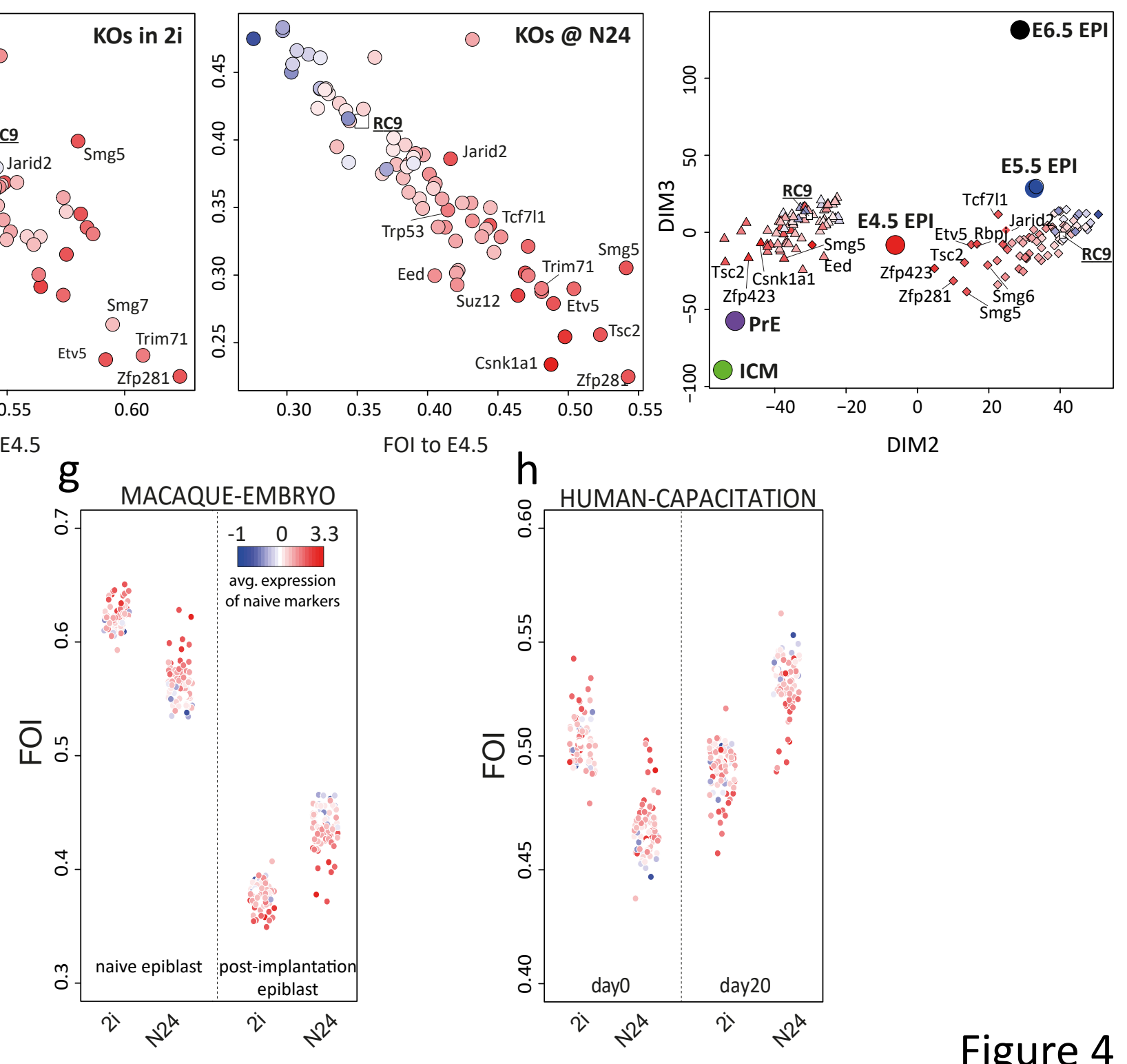

$\mathrm{h}$

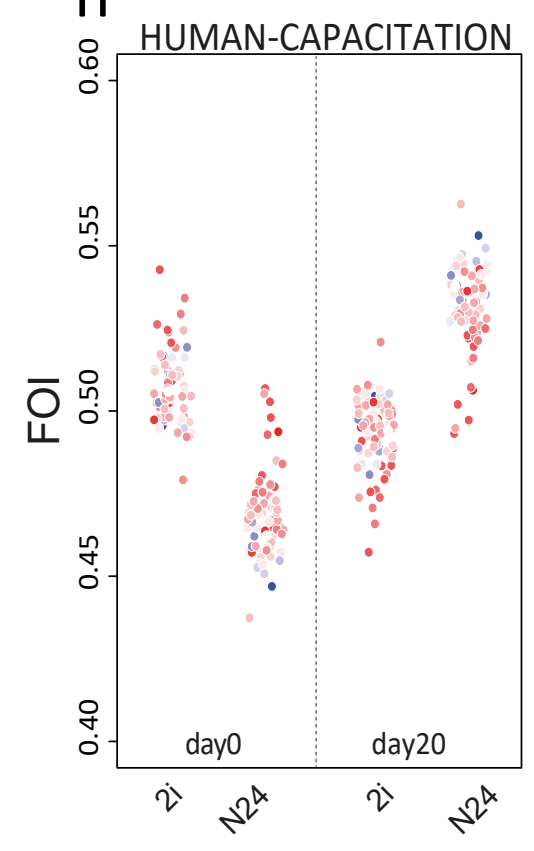

Figure 4 
bioRxiv preprint doi: https://doi.org/10.1101/2020.03.23.000109; this version posted July 2, 2020. The copyright holder for this preprint (which was not certified by peer review) is the author/funder. All fights reserved. No reuse allowed without permission.
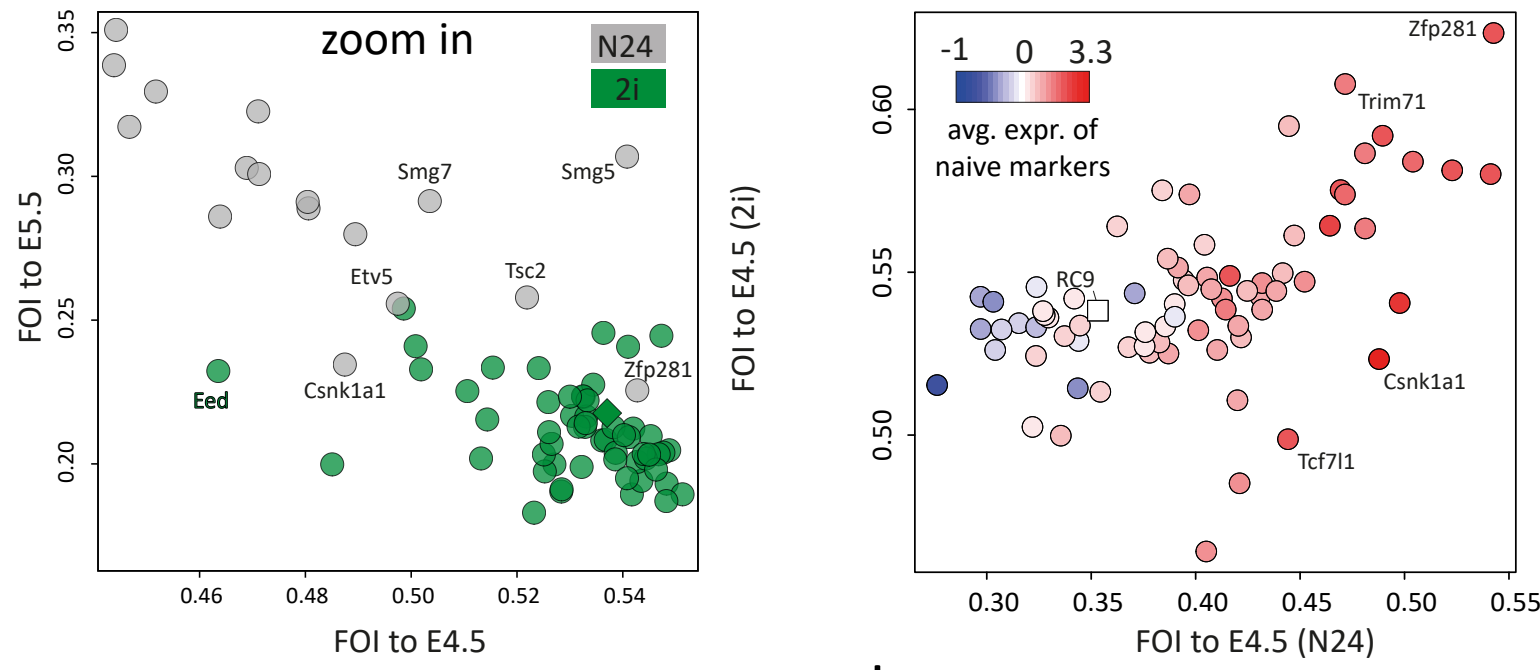

C
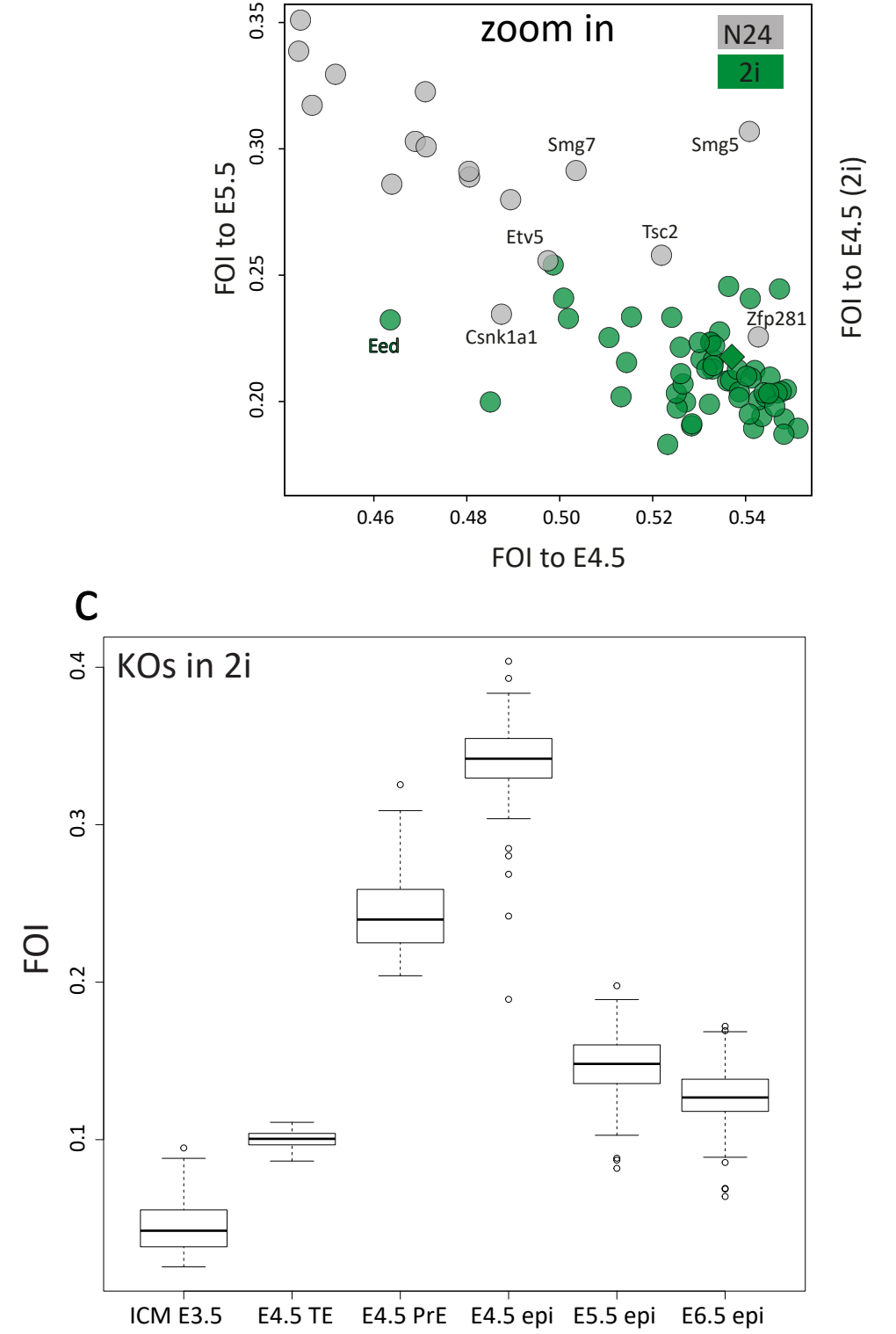

d

e

\section{f}

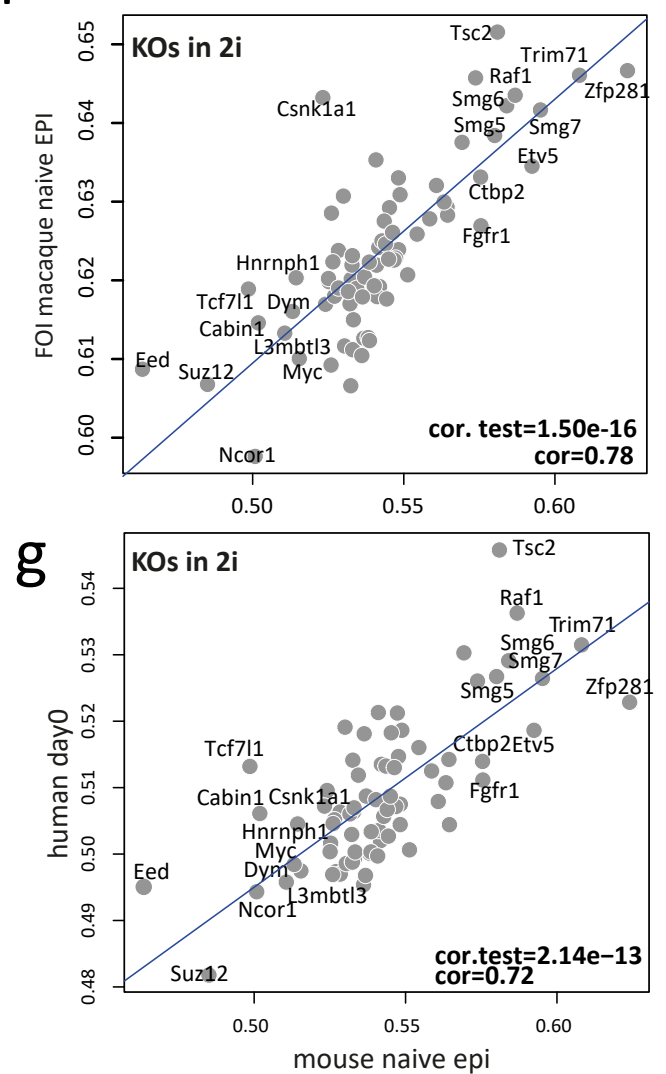

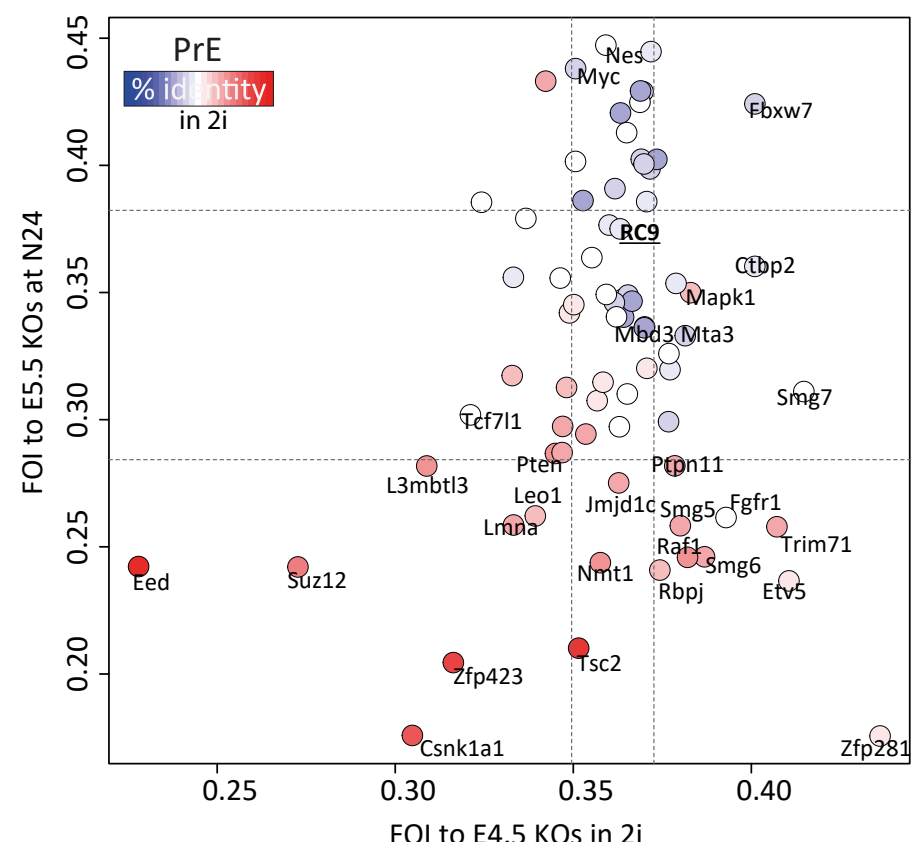

h
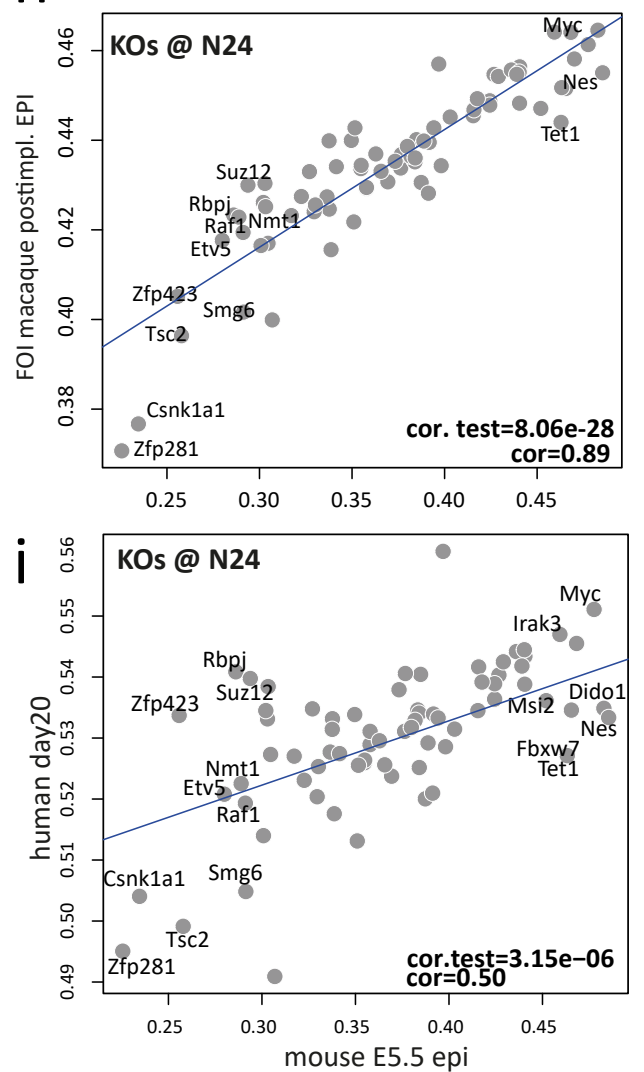
bioRxiv preprint doi: https://doi.org/10.1101/2020.03.23.000109; this version posted July 2, 2020. The copyright holder for this preprint (which was not certified by peer review) is the author/funder. All rights reserved. No reuse allowed without permission.

b
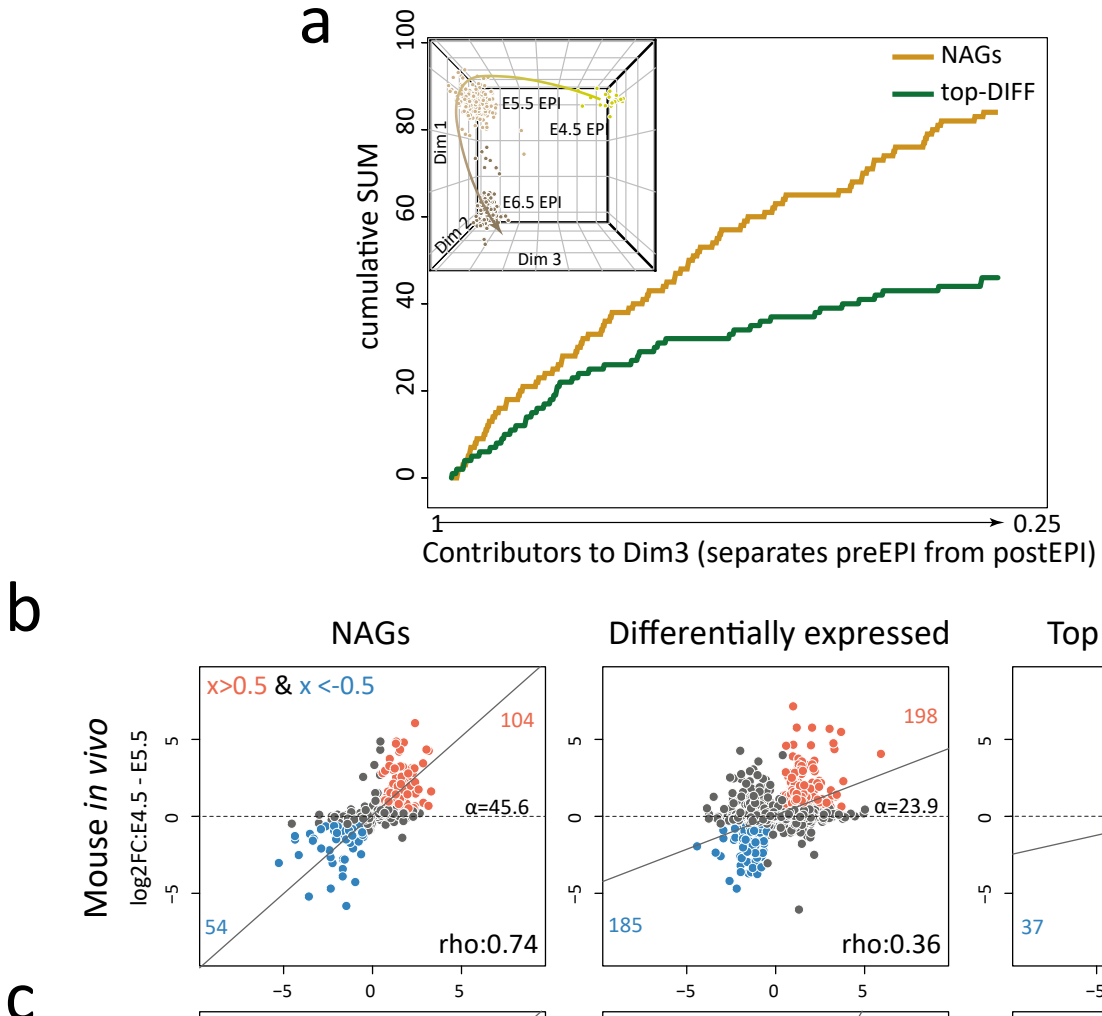

\section{Differentially expressed}

Top diff. expressed

C
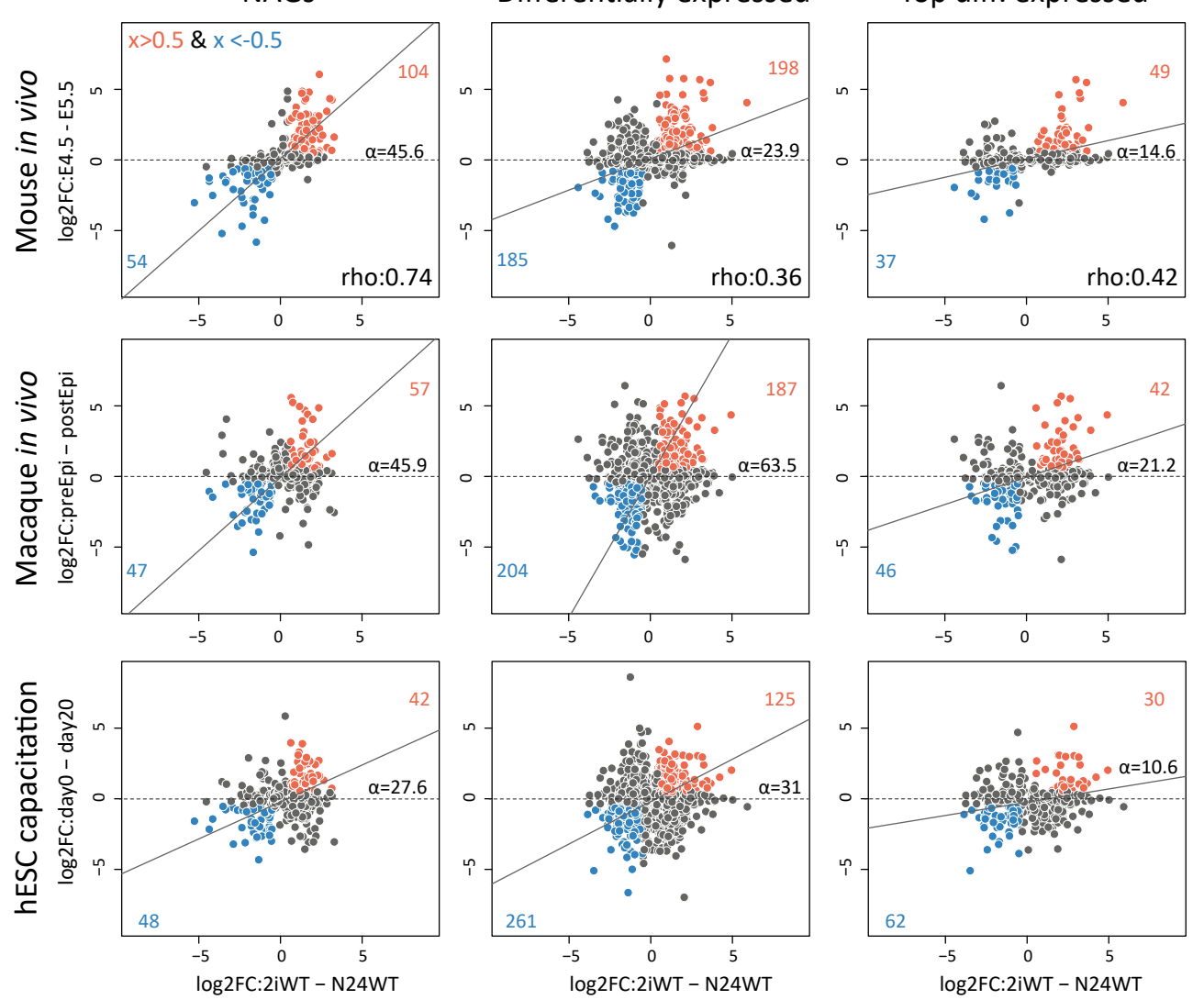



0.0

e Mouse Macaque Human

in vivo in vivo capacitation

adj. $p<0.0001$
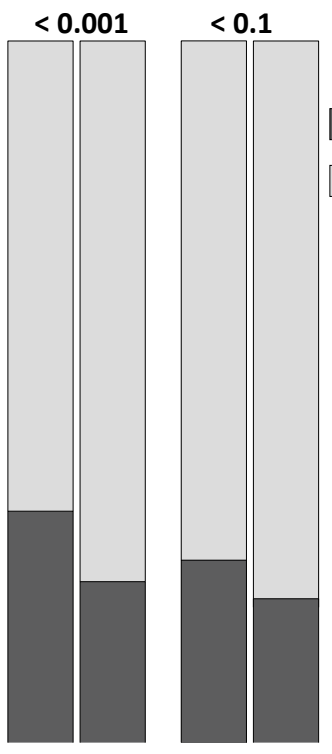

1.0
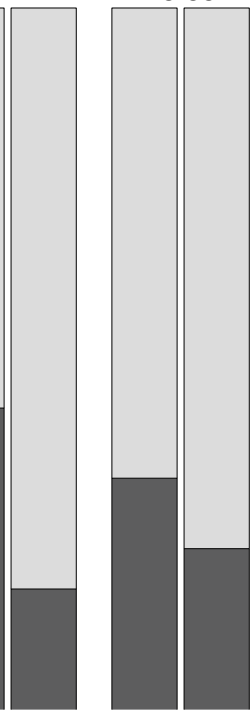

co-regulated

low-change or not co-regulated

$f$

NAGS

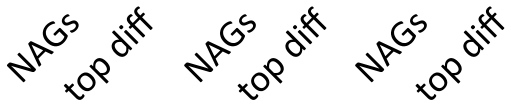
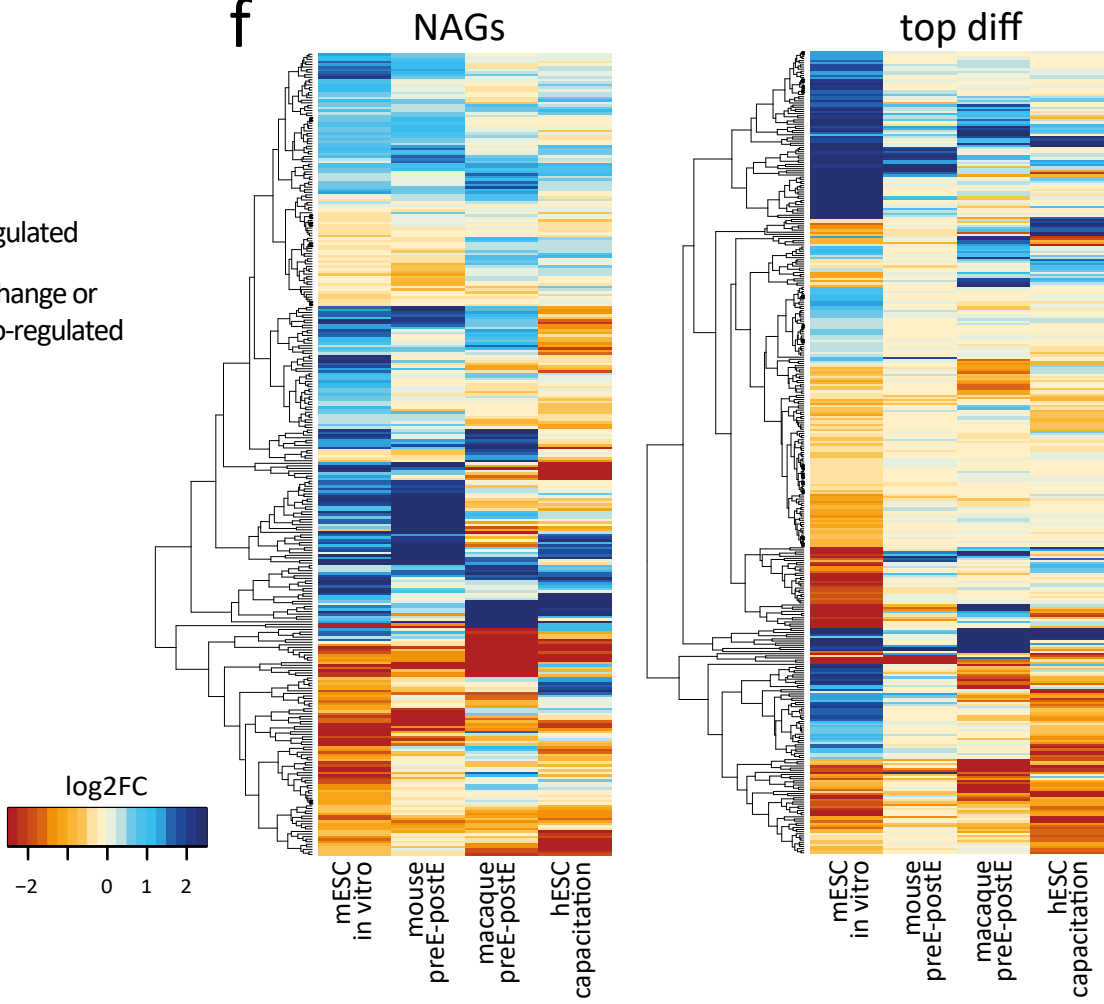

Figure 5 
a

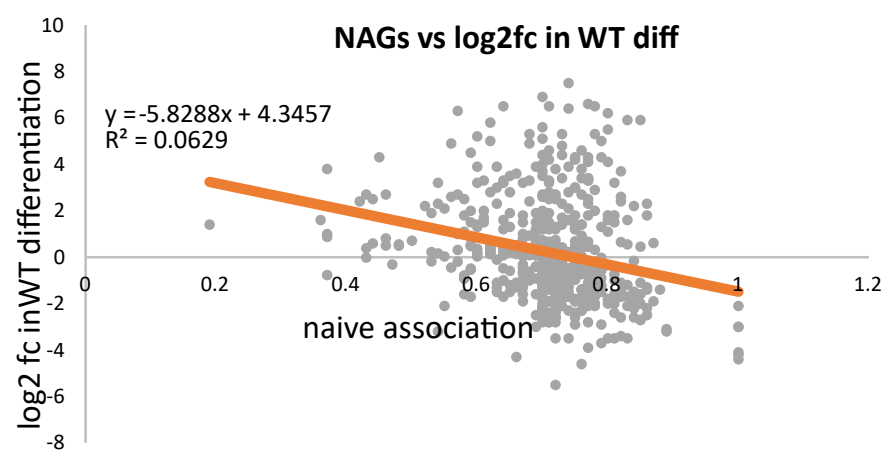

C
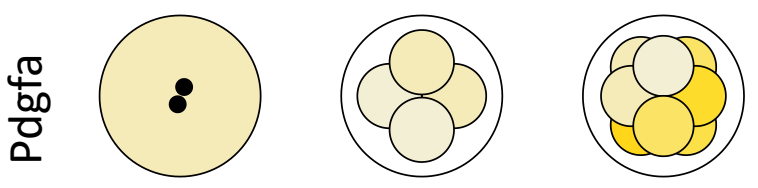

zygote

4-cell

8-cell
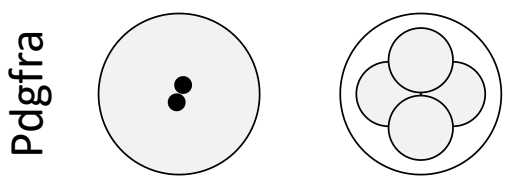

zygote

4-cell

8-cell

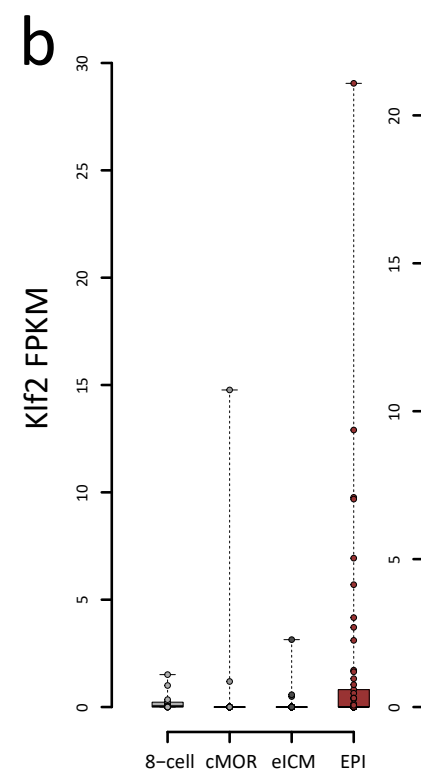

HUMAN
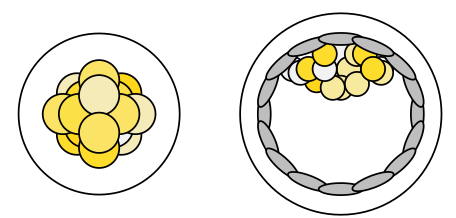

comp.Mor early ICM

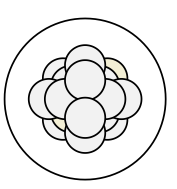

comp.Mor

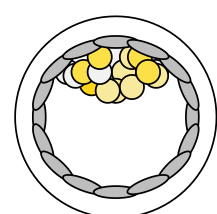

early ICM

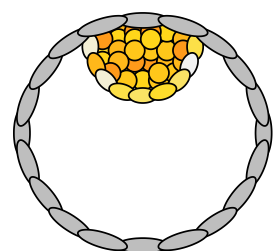

late ICM

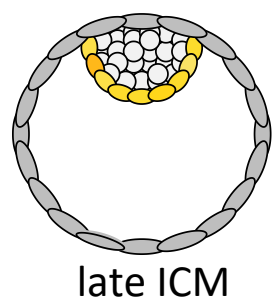

d

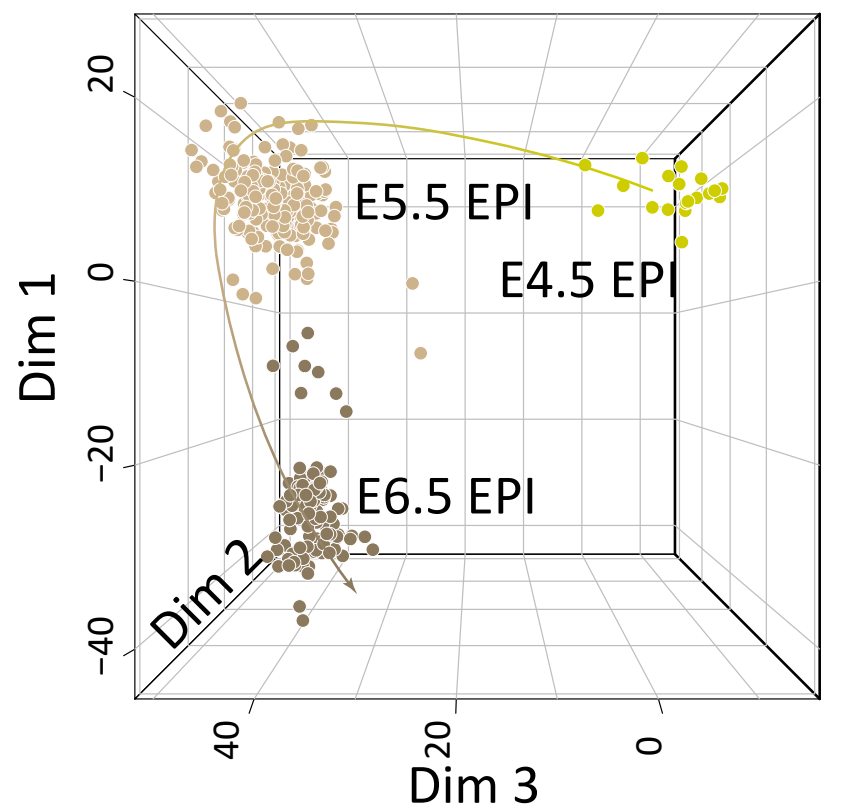


bioRxiv preprint doi: https://doi.org/10.1101/2020.03.23.000109; this version posted July 2, 2020. The copyright holder for this preprint (which was not certified by peer review) is the author/funder. All rights reserved. No reuse allowed without permission.

a

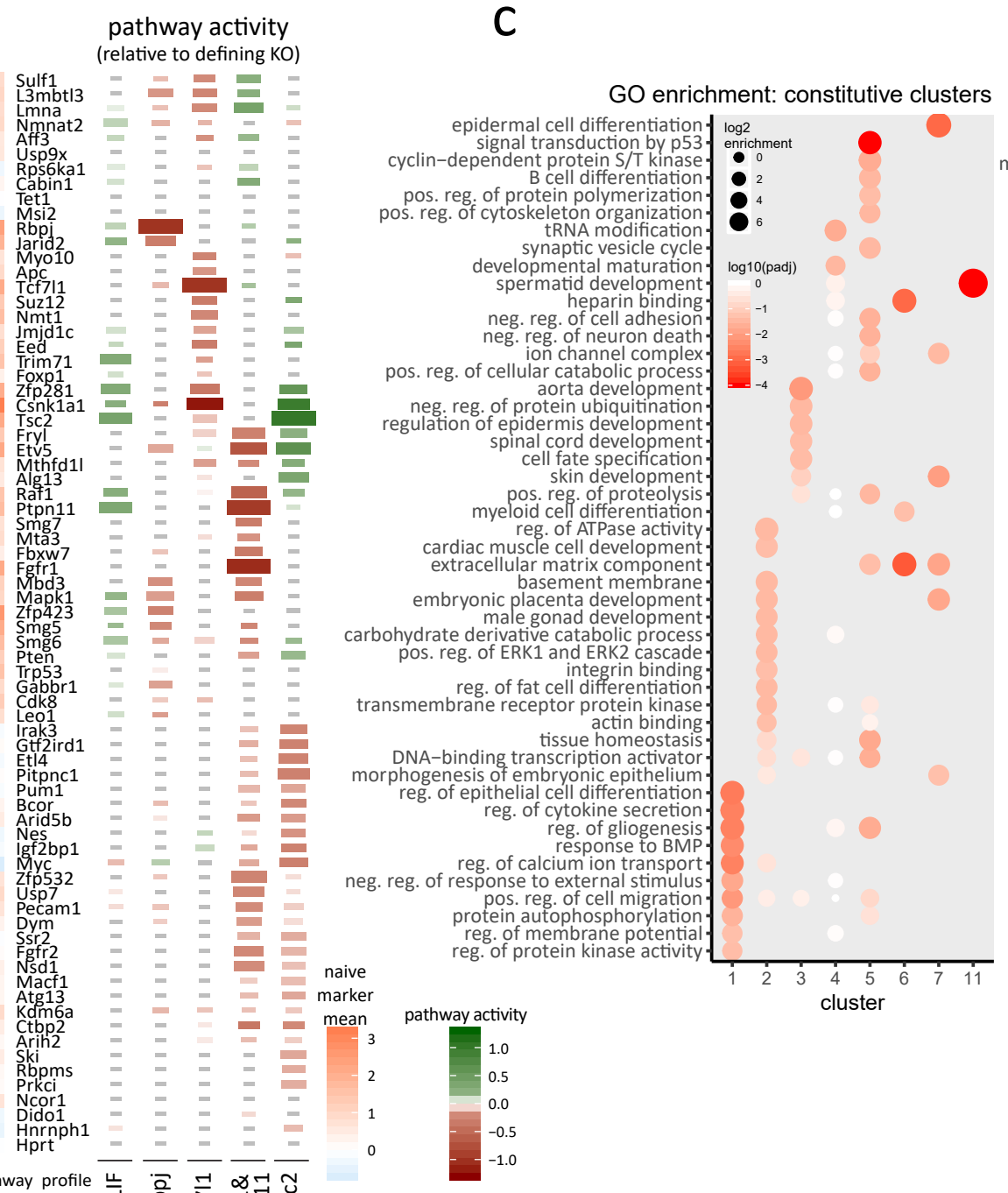

e

GO enrichment: induced clusters

reg. of protein import into nucleus $f$ log 2 morphogenesis of a branching en anithenesis. dendrite development
reg. of membrane potential type eye development T cell differentiation axon guidance
telencephalon development. canonical Wht signaling pathway skeletal system morphogenesis morphogenesis of a branching structure maintenance of location epithelial tube morphogenesis transcription activator activity reg. of developmental growth ameboidal-type cell migration reg. of innate immune response. pos. reg. of protein secretion pos. reg. of protein secretion
cytosolic ribosome cytosolic ribosome focal adhesion
mRNA binding mammary gland development actin-based cell projection mesenchymal cell differentiation neg. reg.n of neurogenesis peptidyl-yysine modification

pos. reg. of secretion by cell
reg. of cell-cell adhesion reg. of cell-cell adhesion stem cell population maintenance plasma membrane receptor complex cell fate commitment response to steroid hormone response to grow th factor stimulation. embryonic organ morphogenesis transcription factor complex reg. of immune effector process
eproductive structure development reg. of apoptotic signaling pathway

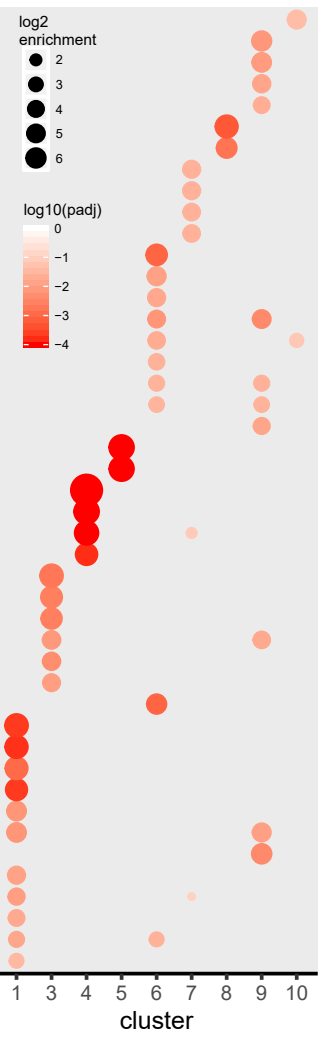

cluster avg. $\log 2 \mathrm{FC}(\mathrm{N} 2)$

4
-2
0

b

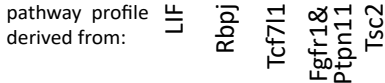

Constitutive KO response (logFC at N24)

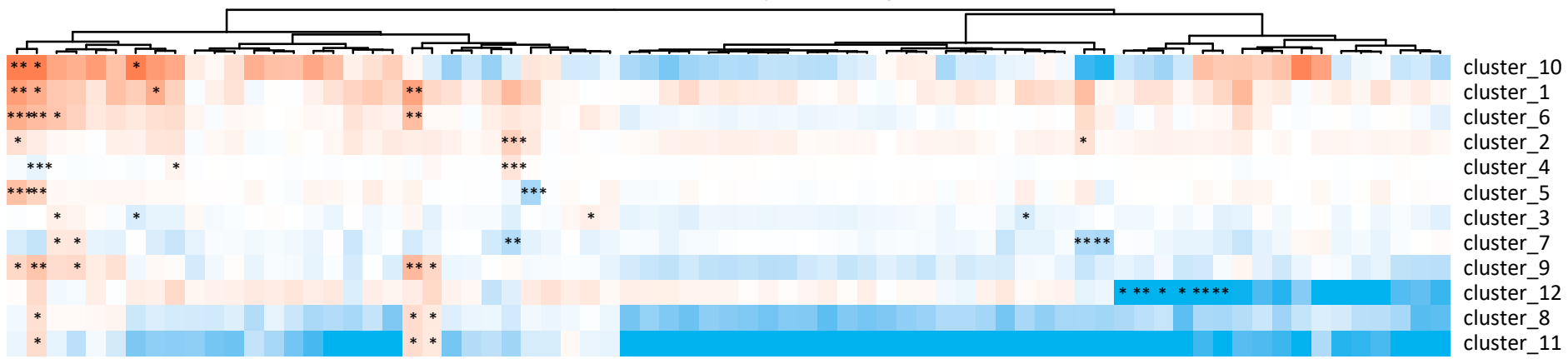

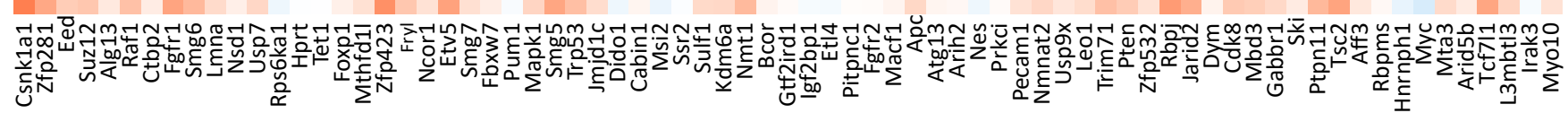

naive markers

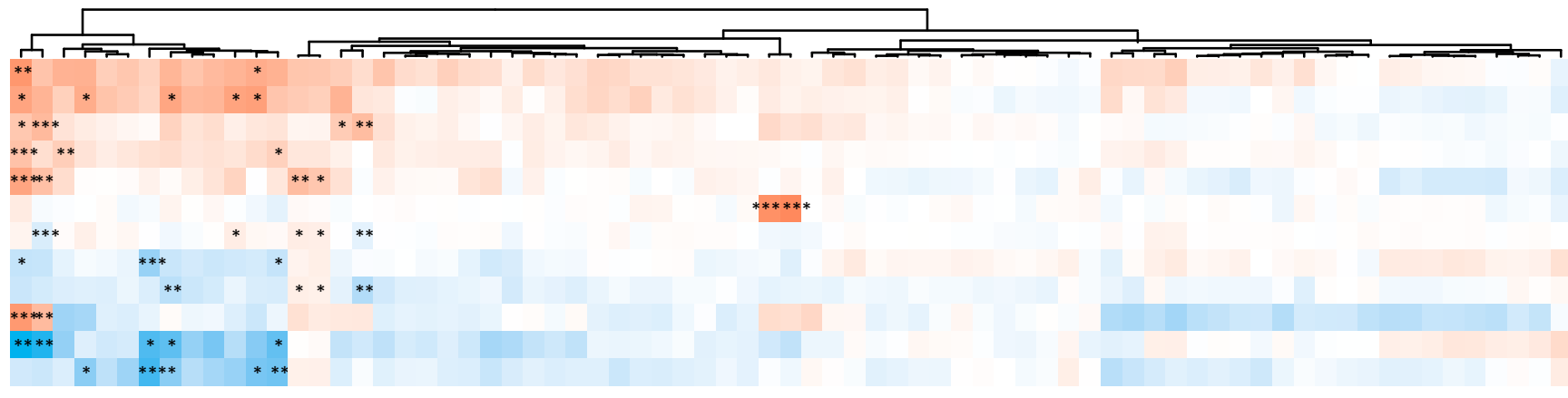

cluster_1

cluster_11

cluster_6

cluster_10

cluster_5

cluster_4

cluster_7

cluster 9

cluster_3

cluster_12

cluster 8

cluster_2

naive markers 
bioRxiv preprint doi: https://doi.org/10.1101/2020.03.23.000109; this version posted July 2, 2020. The copyright holder for this preprint (which was not certified by peer review) is the author/funder. All rights reserved. No reuse allowed without permission.

a Example pathway signature workflow, Tsc2 (mTOR signalling)

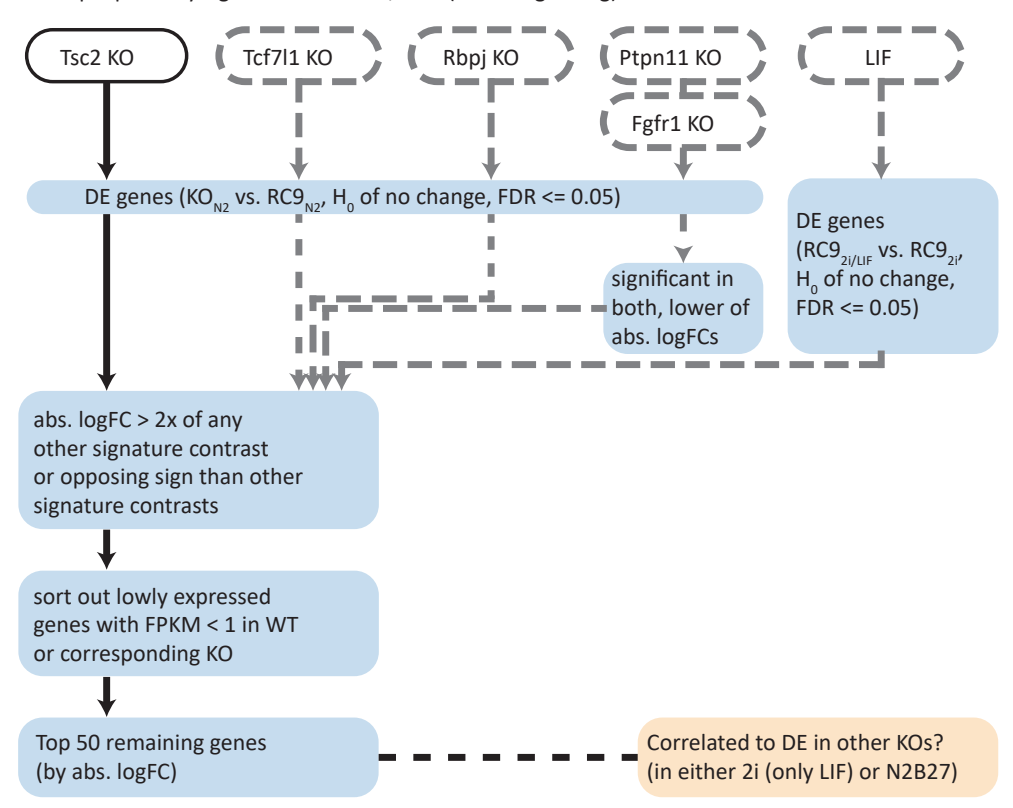

b

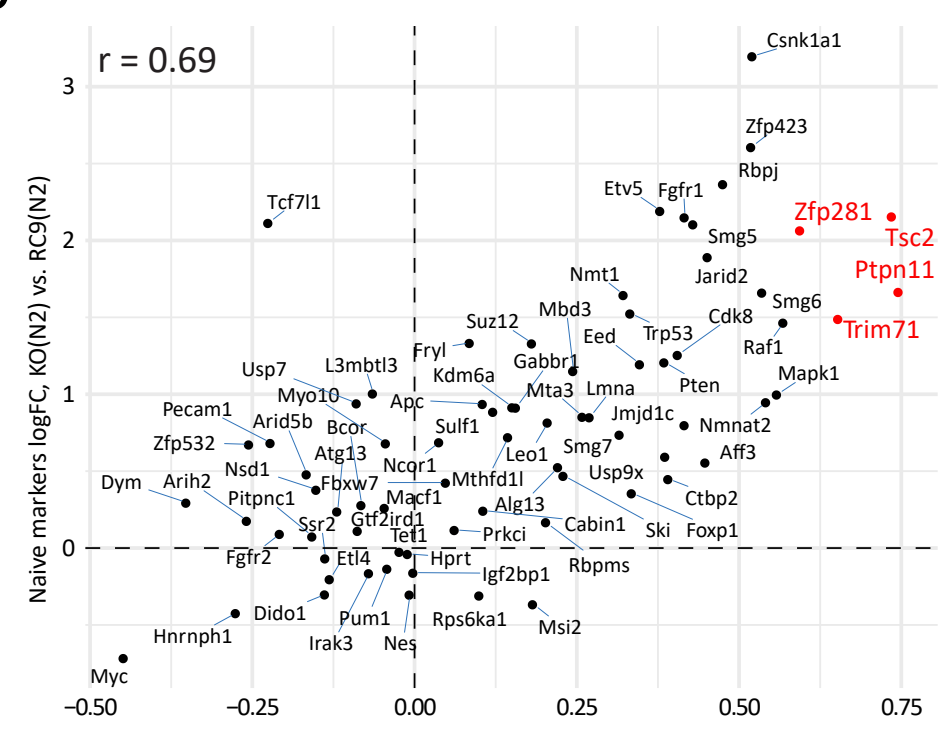

correlation $[\mathrm{KO}(2 \mathrm{i})$ vs. $\mathrm{RC9}(2 \mathrm{i})] \sim[\mathrm{RC}(2 \mathrm{iL})$ vs. $\mathrm{RC9}(2 \mathrm{i})]$

e

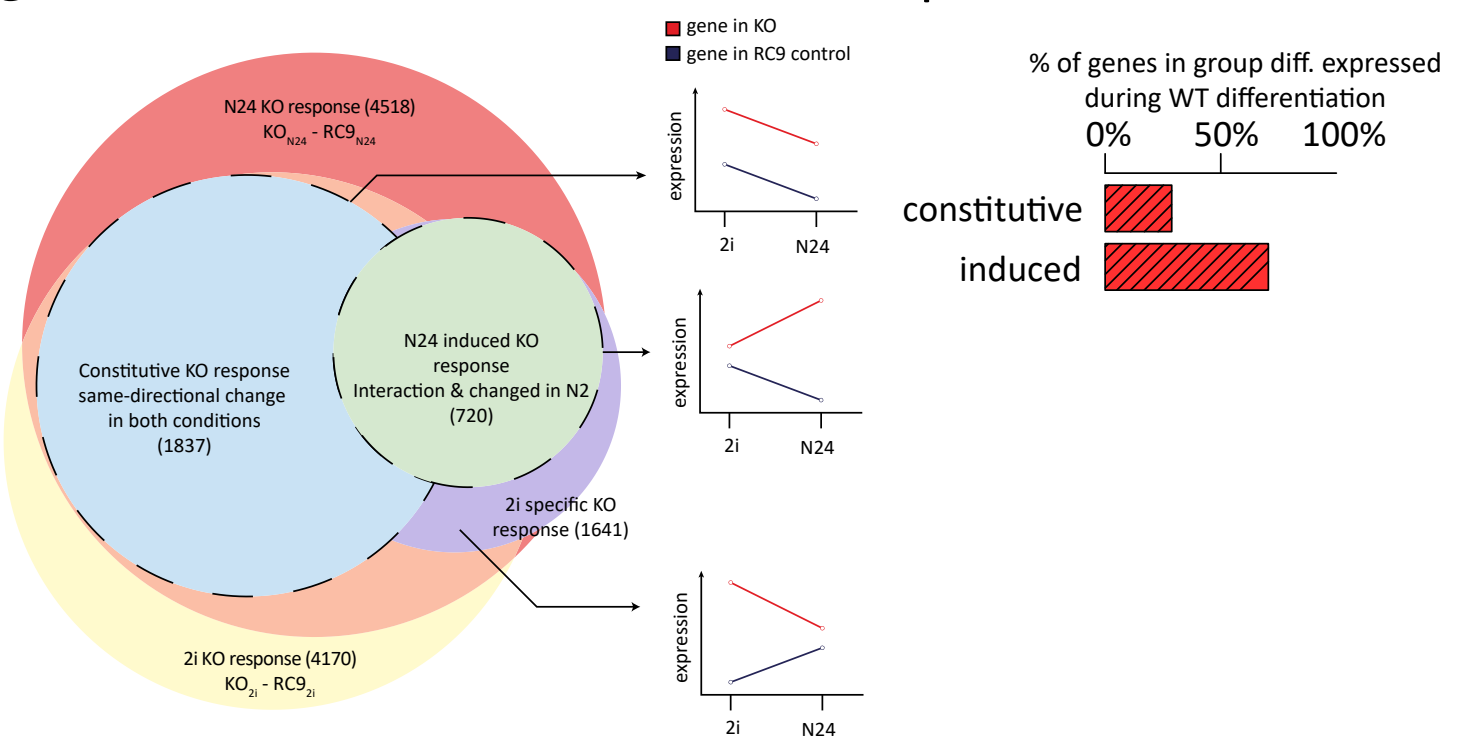

C

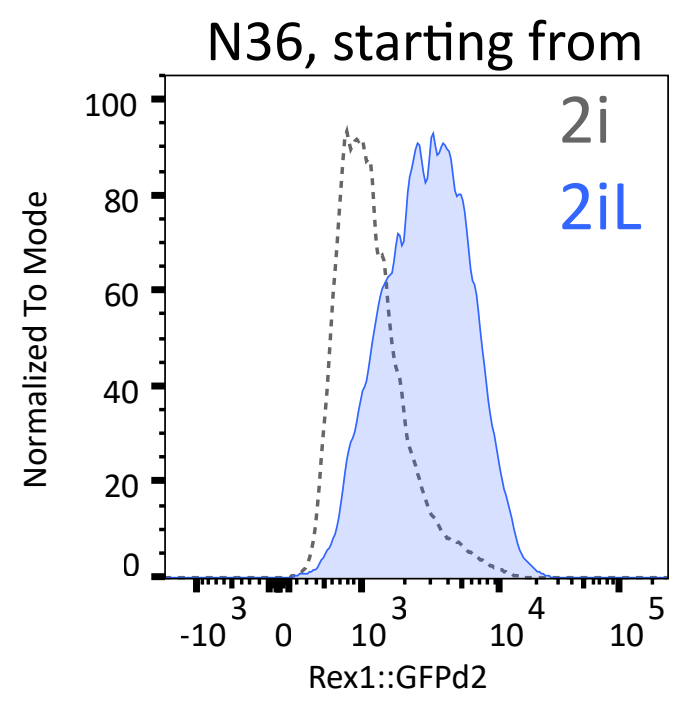

Predictiveness of pathway perturbation for strength of differentiation delay phenotype

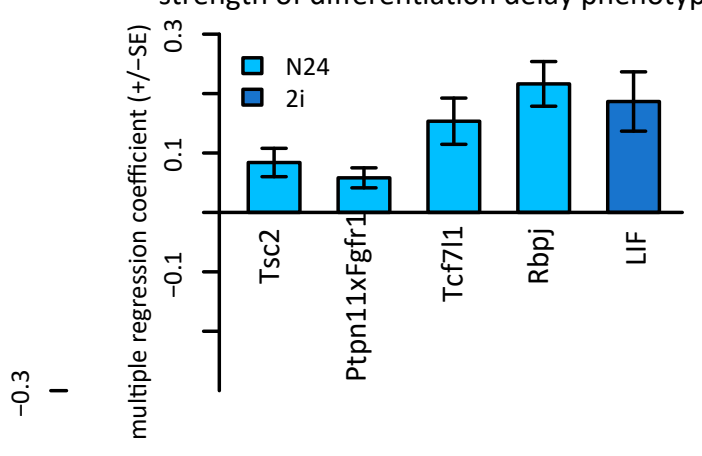

\section{Supplementary Figure 6}




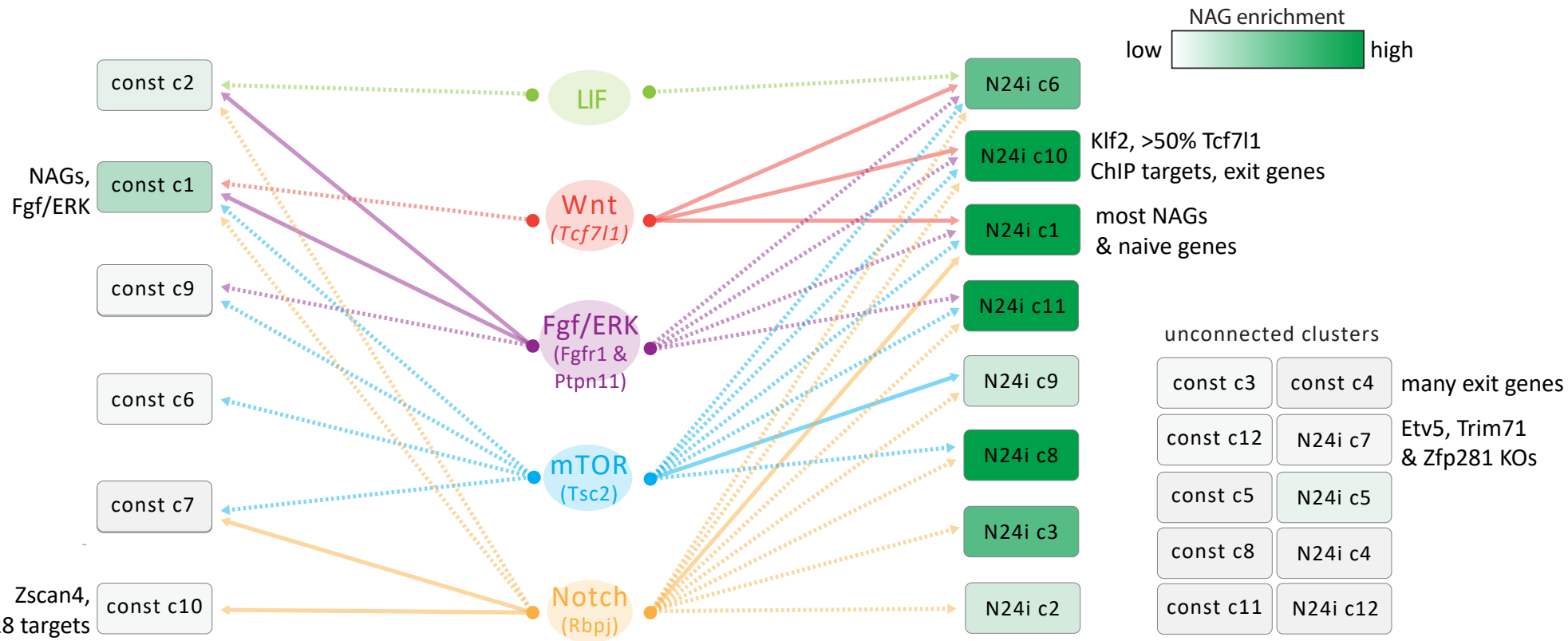

b

naïve GRN on

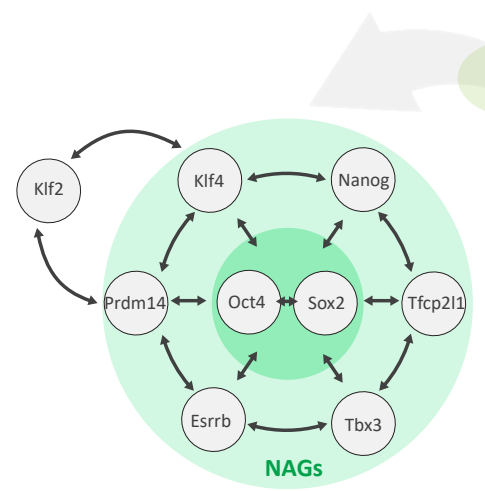

naïve

in vitro

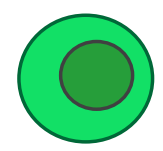

or

in vivo

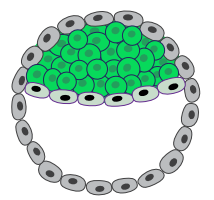

LIF

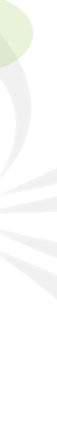

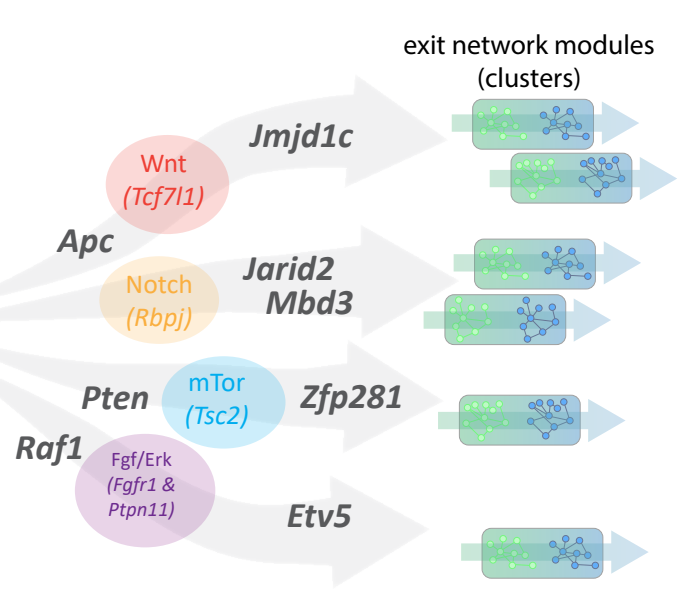

exit network modules

formative GRN on

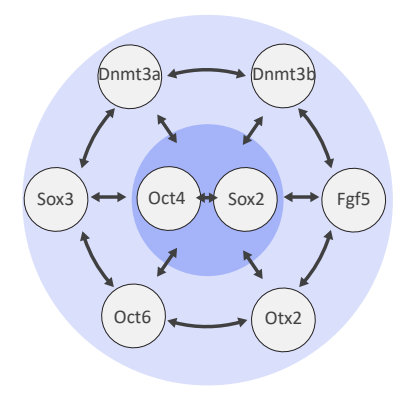

\section{formative}

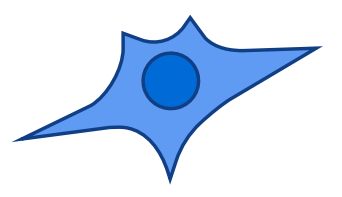

(e.g. Zfp281, Smg5)

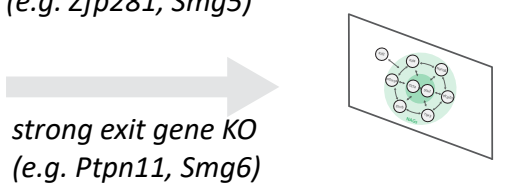

(e.g. Ptpn11, Smg6)

intermediate exit gene $K O$

(e.g. Nmt1, Cdk8)

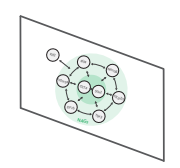

weak exit gene $K O$

(e.g. Jmjd1c, Leo1)
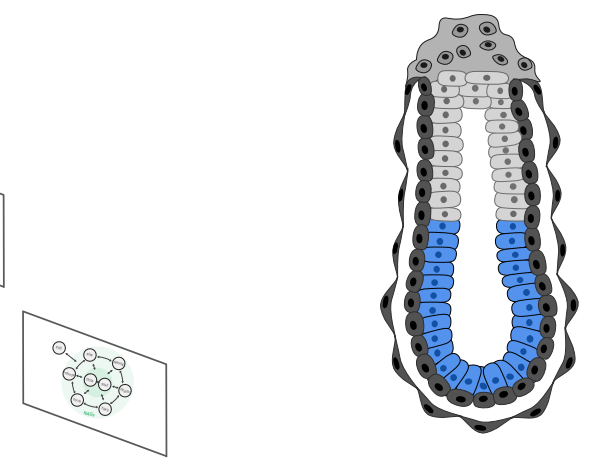


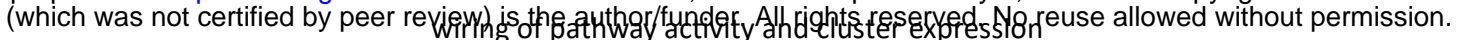

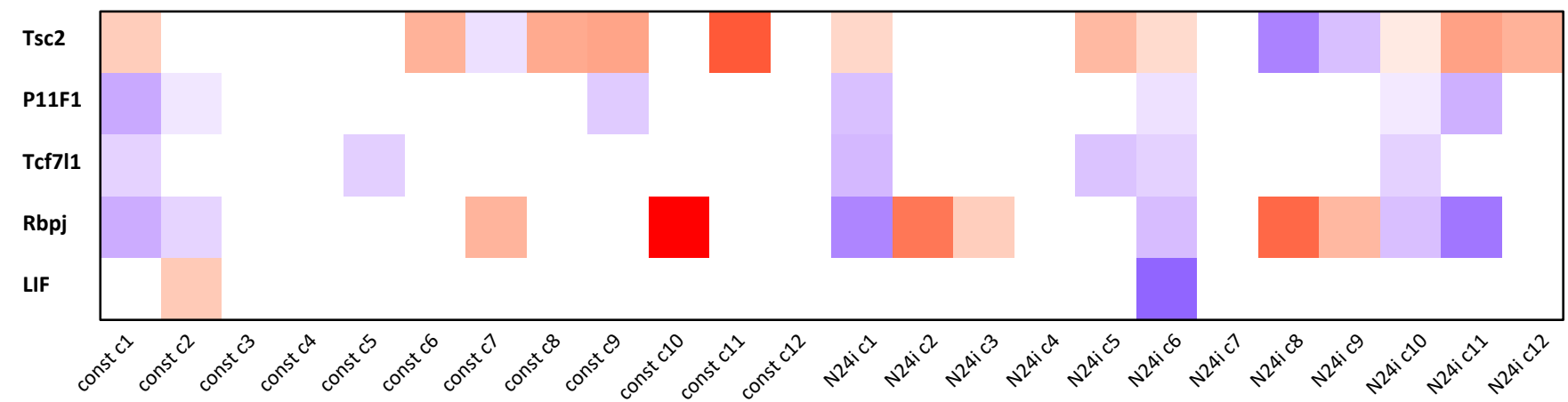

b

pathway activity over time course

- avg. $\log \mathrm{FC}$ relative to mean

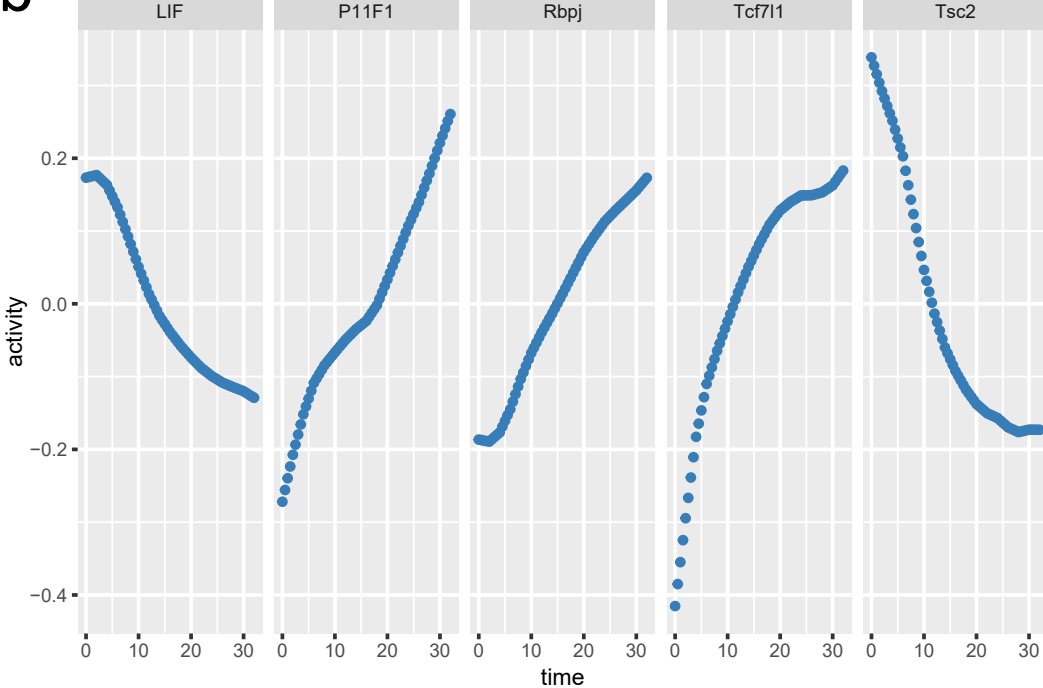

C predicted kinetics based on pathway activity (induced clusters)

.

\begin{tabular}{l|l|l|l|l|l|l|l|l|l|l|l|l|l|l|l|l|l} 
cluster1 & cluster2 & cluster5 & cluster8 & cluster9 & cluster10 & cluster11 & cluster3 & cluster6 & cluster12 & cluster4 & cluster7
\end{tabular}
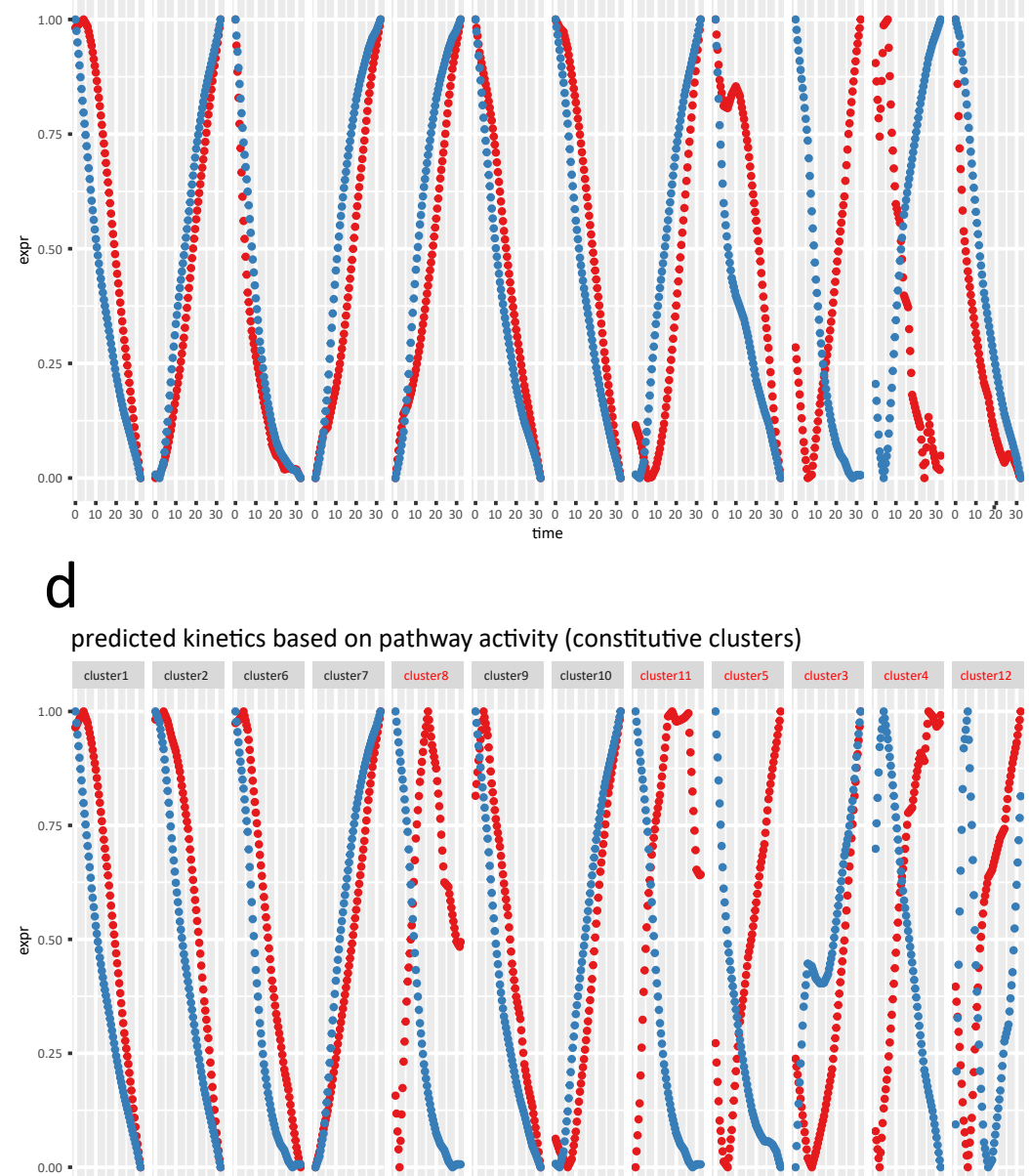

d

time

\section{Supplementary Figure 7}

e
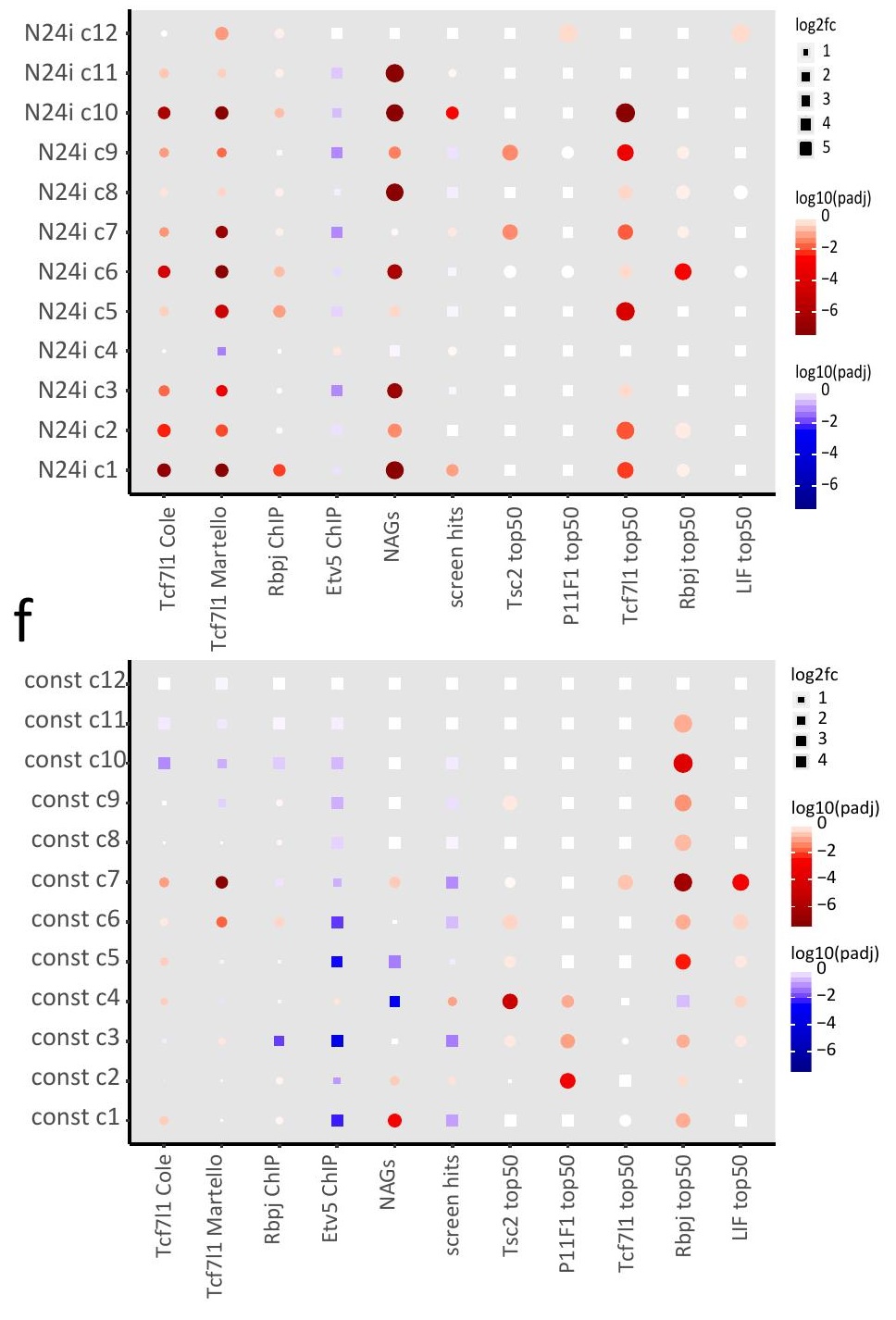


\section{Figure Legends}

\section{$595 \quad$ Figure 1}

596 a, Illustration of the Rex1::GFPd2 reporter cell line and its' exit from naïve pluripotency. Rex1-

597 GFPd2 (in short Rex1-GFP) expression is tightly linked to naïve pluripotency. Shutdown of GFP expression indicates commitment to differentiation.

599 b, FACS analysis of Rex1-GFPd2 reporter levels throughout a 72h differentiation time-course after $2 \mathrm{i}$ withdrawal.

601

c, Scheme of the screening strategy to identify candidate genes involved in exit from naïve pluripotency. After random insertional mutagenesis using piggyBac transposon gene-traps, haploid Rex1::GFPd2 ESCs were released into differentiation. Cells that maintain GFP expression after exposure to differentiation conditions were isolated and the gene-trap insertion sites mapped.

d, Plot showing cumulative number of hits and the cumulative number of novel hits over 35 independent insertional mutagenesis screens in haploid ESCs.

e, Representative Rex1-GFP FACS plots showing the differentiation delays $30 \mathrm{~h}$ after $2 \mathrm{i}$ withdrawal of Tcf7l1, Trim71 and Mbd3 KOs. A Myc KO served as a negative control. Blue

610 indicates the KO FACS profiles and dashed lines indicates WT Rex-GFP.

611 f, Differential expression of genes at N24 vs. 2i in WT RC9 cells. Black dots show significance

612 (FDR $\leq 0.05, \mathrm{H0}:|\mathrm{FC}|>1.5)$. Pluripotency genes are red dots, haploid screen hits are blue dots, the 73 KO-genes are green dots.

$614 \mathrm{~g}$, t-SNE projection of the $73 \mathrm{KOs}$ in 2i, based on expression of 3058 differentiation-associated 615 genes $\left(\mathrm{RC9}^{\mathrm{N} 24}\right.$ vs. RC92i, $\left.\mathrm{FDR} \leq 0.05, \mathrm{HO}:|\mathrm{FC}|>1.5\right)$. Differentiation defects observed at N24 616 in the respective KOs are indicated by a colour gradient, measured as average naive marker 
618 in the respective KO at N24. Red: delayed differentiation; blue: accelerated differentiation 619 see key in $\mathbf{h}$.

620 h, Similar to g, for KOs at N24

621

\section{Supplementary Figure 1}

623 a, The top 15 candidate genes from the haploid screen ordered by the number of times the 624 hit was found in the 35 screens, including the number of independent integration sites and 625 the calculated FDR.

b, Significantly enriched pathways among screen hits ranked according to fold enrichment; colours represent FDR in screen analysis. Numbers next to bars indicate number of hit-genes within category,

c, Significantly enriched GO terms among screen hits as in $\mathbf{b}$,

e, Idealized strategy illustrating gRNAs and genotyping primers.

632 f, Western analysis using indicated KOs and indicated antibodies. Tubulin or Gapdh were used as loading controls, as indicated.

g, Anti-flag specific Westerns in indicated KO ${ }^{\text {rescue }}$ ESCs upon stable forced expression of 3xflag rescue cDNAs driven from a CAG promoter.

h, Rex1GFP levels measured by FACS showing restoration of differentiation behaviour in the indicated rescue cell lines at N30.

$638 \mathrm{i}, \mathrm{t}-\mathrm{SNE}$ visualization of RNA-seq profiles in $2 \mathrm{i}$ and at N24 before and after batch correction. expressed between $2 \mathrm{i}$ and N24 in WT ESCs (FDR $\leq 0.05, \mathrm{HO}:|\mathrm{FC}|<1.5)$. 
642 (Ifc $\geq 0.8 ;$ adj. $p$-value $\leq 0.05)$. Log2FC, adj. $p$ value and quantification of the molecular 643 phenotype using the average upregulation of a set of naïve marker genes (Esrrb, Nanog, $644 T f c p 2 / 1, T b \times 3, \operatorname{Prdm14}, K l f 4)$ are shown.

645 I, Dot-plot showing regression analysis of KO-induced changes at N24, comparing Tcf7l1 KO to Apc and Csnk1a1 KOs. Total least squares (TLS) analysis results are indicated.

$647 \mathrm{~m}$, Rex1-GFP levels measured by FACS at N24 showing the effect of Epiblastin A addition during differentiation.

649

n, Rex1GFP analysis at N24 of Pum1 KO cells, showing a relatively strong differentiation defect at early passages.

651

652

Figure 2

653

a, Naïve (top) and formative (bottom) marker gene expression changes at N24 compared to

654 WT in all 73 KO ESCs. Clustering based on naïve marker gene expression shows a wide 655 distribution of differentiation delay phenotypes.

656

b, Rex1GFP FACS analysis of Jarid2 and Tcf7/1 KO ESCs after transfection with negative control or Klf2 specific siRNAs.

c, For each of the $73 \mathrm{KOs}$, the change of mean naïve marker gene expression at N24 (KO vs WT) was plotted against the change of the global transcriptome at N24 state (correlation of

660 KO-induced change at N24 with differentiation-induced changes in WT differentiation, N24 vs 661 2i).

d, Comparison of number of genes deregulated in $2 \mathrm{i}$ and at N24 in all KOs (FDR $\leq 0.05, \mathrm{HO}$ : $|F C|<1.5)$. Differentiation phenotypes are colour coded according to mean naïve marker gene expression change in KOs at N24. 

the Gsk3 inhibitor, $\mathrm{CH}$.

667

668

\section{Supplementary Figure 2}

669

a, RNA-seq based fold changes (FC, relative to $W T$ ) of indicated naïve marker genes in indicated KOs in $2 \mathrm{i}$ (only FCs with adj. $\mathrm{p} \leq 0.05$ are shown).

671 b, RNA-seq derived data showing FCs of indicated formative marker genes in indicated KOs in 672 2i (only FCs with adj. $p \leq 0.05$ are shown).

673 c, Rex1-GFP analysis of WT cells at N24, transfected with negative control or KIf2 specific siRNAs. KIf2 depletion increases the speed of Rex1GFP downregulation.

675 d, Comparison of the number of differentially expressed genes at N24 (FDR $\leq 0.05, \mathrm{HO}$ : $|\mathrm{FC}|$ $<1.5)$ to the mean naïve marker gene expression at N24 (phenotype strength). Stronger differentiation phenotypes correlate with more differentially expressed genes.

e, Expression log2 fold changes (log2FC) at N24 between knockout and RC9 control, comparing Tcf7l1 KOs to Jmjd1c KOs. Each dot corresponds to one gene. Only genes showing significance (FDR $\leq 0.05, \mathrm{HO}:|\mathrm{FC}|<1.5)$ in either one of the KOs are plotted. Red line: total genes is shown by largely identical directionality of regulation of significantly regulated genes in both KOs.

Figure 3

a, Scheme illustrating comparisons of the $73 \mathrm{KO}$ gene expression profiles at N24 with a $2 \mathrm{~h}$ resolution in vitro differentiation time course of WT RC9 ESCs. 
b, Heatmap showing differentiation delays of the $73 \mathrm{KO}$ lines quantified by average naïve marker gene expression. Red bars indicate closest correlation to that specific time-point. Positive values indicate delayed differentiation. Negative values more rapid differentiation.

691 Each line corresponds to one KO. Selected KOs are indicated. See Supplementary Table 2 for 692 the full hierarchy of the 73 KOs. 3058 genes changed in WT differentiation from $2 i$ to N24.

\section{Supplementary Figure 3}

697

a, Principle component analysis (PCA) of the $2 \mathrm{~h}$ resolved WT differentiation time course sampled by RNA-seq. Oh corresponds to cells in $2 \mathrm{i}$.

b, Heatmap showing differentiation delays of the $73 \mathrm{KO}$ lines quantified using expression of all 3058 genes differentially expressed in WT between $2 \mathrm{i}$ and N24. Red bars indicate closest correlation to that specific time-point. Positive values indicate delayed differentiation. Negative values more rapid differentiation compared to WT. Each line corresponds to one KO. Selected KOs are indicated.

Figure 4

a, Scheme illustrating the comparison of gene expression profiles of $73 \mathrm{KO}$ in $2 \mathrm{i}$ and at N24 with in vivo derived averaged single cell expression data comprising peri-implantation epiblast tissues. Specifically, transcription profiles of each $\mathrm{KO}$ in $2 \mathrm{i}$ and at N24 were compared with in expression datasets obtained from the E4.5, E5.5 and E6.5 epiblast. 
represents one KO. Average naïve marker expression in KOs at N24 is indicated by a colour

713 gradient.

714

c, Plot showing a comparison of FOls of WT (RC9) and KO cells in 2i (green) and at N24 (grey) to E4.5 and E5.5 mouse epiblast, computed using all expressed genes ( $\log 2$ FPKM $>0)$.

716 (d,e) Data shown in Figure 4c separated by culture condition. Comparison of FOls of WT and

717 KO cells in $2 \mathrm{i} \mathrm{d}$, and at N24 e, to E4.5 and E5.5 mouse epiblast; average naïve marker 718 expression in KOs at N24 is indicated by a colour gradient.

f, PCA showing all KOs in $2 \mathrm{i}$ (triangles) and at N24 (diamonds) and embryo derived datasets (ICM, PrE, E4.5Epi, E5.5Epi, E6.5Epi). As in d,, blue to red colour gradient indicates naïve marker expression at $\mathrm{N} 24$ of the respective KO.

g, Similar to Figure $4 b$, showing FOls between $2 \mathrm{i}$ and N24 datasets and macaque pre- and post-implantation embryos.

h, Similar to Figure 4b, showing FOls between $2 \mathrm{i}$ and N24 datasets and hESC capacitation datasets comprising hESCs in naïve conditions (day0) and hESCs 20 days after release into primed conditions (day20).

\section{Supplementary Figure 4}

a, Zoom in of the plot in Figure 4c, focusing on the overlap of several KOs in $2 \mathrm{i}$ (green) and in N24 (grey). N24 samples of Etv5, Smg7, Tsc2, Smg5 and Zfp281 KOs show FOls to the E4.5 EPI

731 similar to KOs cultured in $2 \mathrm{i}$.

b, Comparison of FOls to the E4.5 epiblast of all KOs in 2i compared to FOI of all KOs at N24; average naïve marker expression in KOs at N24 is indicated as colour gradient. With some exceptions, stronger in vitro phenotypes translate to increased similarity to the E4.5 epiblast in $2 \mathrm{i}$ and at $\mathrm{N} 24$. 

epiblast (Epi), E5.5 Epi and E 6.5 Epi. Analysis extended but similar to Figure 4b. epiblast(y-axis). Dotted grey lines indicate 95\% confidence intervals. Colour gradient shows

740 similarity of $2 \mathrm{i}$ expression profiles to PrE.

741 e, Correlation plot of FOls between 2i profiles and mouse naïve (E4.5) epi and FOls between

742 2i profiles and macaque naive epi (correlation significance is indicated).

$743 \mathbf{f}$, Correlation plot of FOls between $2 \mathrm{i}$ profiles and mouse naive epi and FOls between $2 \mathrm{i}$

744 profiles and human naïve ESCs (day0) (correlation significance is indicated). profiles and macaque post-implantation epi (correlation significance is indicated). profiles and human ESCs at day 20 after release into primed culture conditions (correlation significance is indicated).

750

\section{$751 \quad$ Figure 5}

752 a, x-axis displays all $3^{\text {rd }}$ dimension (Dim3) genes, which separates mouse naïve from post753 implantation epiblast (the inset shows the full PCA plot, see also Supplementary Figure 5d) in order of extent of contribution to Dim3. The contribution to Dim3 of NAGs (orange) and the top 250 differentially regulated genes (green) are plotted in a cumulative manner.

b, Plots comparing relative log2 FC in mouse ESC differentiation ( $2 \mathrm{i}$ vs $\mathrm{N} 24$ ) to relative $\log 2 \mathrm{FC}$ in mouse in vivo transition from pre- to post-implantation epiblast (E4.5 vs E5.5). Selected gene groups are plotted: i) NAGs, ii) all 3058 differentially expressed genes in mESC 
values indicate the level of correlation between in vivo and in vitro differentiation of given gene groups. Alpha indicates the angle between the $x$-axes and the orthogonal regression. Genes with $\log 2 \mathrm{FC}>0.5$ and $<-0.5$ in both the $\mathrm{x}$ - and $\mathrm{y}$-axes are highlighted in orange and blue respectively. macaque in vivo transition from pre- to post-implantation epiblast cells. naïve ESC capacitation ( $\mathrm{d} 0$ to $\mathrm{d} 20$ ).

e, Graphs showing the frequency of co-regulation of NAGs or the top 250 differentially expressed genes ( $2 \mathrm{i}$ vs N24) with differentially expressed genes in mouse and macaque in vivo pre- to post-implantation epiblast differentiation, and human naïve ESC capacitation. Twotailed chi-square test ( $\mathrm{Cl}$ 95\%) was used to compute significance levels between the expected and the observed number of modulated genes.

f, One-way hierarchical clustering showing expression changes of NAGs (left) and top differentially expressed genes (right) in the indicated differentiation models.

\section{Supplementary Figure 5}

777 a, Expression change of NAGs during naïve to formative differentiation in WT ESCs (y-axis) versus the strength of link to the naïve network (naïve association, $x$-axis). The orange line

779 indicates the linear regression. The $R^{2}$ value is indicated in the plot.

780 b, KIf2 expression at the indicated stages. Compacted morula (cMOR), expanded ICM (eICM), mouse preimplantation development. 
c, Plots derived from the GRAPPA visualisation-app showing expression in yellow of Pdgfa and its cognate receptor $P d g$ fra during mouse preimplantation embryo development.

a, Relationship between the KO expression profiles at N24 (or in 2i for the LIF pathway) to the expression footprints of the five signalling pathways as indicated (see also Supplementary Figure 6a). Relative pathway activity is -1 for Fgf/ERK (defined by Fgfr1 \& Ptpn11 KOs), Wnt (Tcf7l1 KO) and Notch (Rbpj KO) and +1 for mTOR (Tsc2) and LIF (defined by presence of LIF in $2 \mathrm{i}$ medium) for effects equally strong as in the representative KO. Pathway activity is indicated by colour code (green for activity, red for inactivity). All tiles with an absolute pathway activity smaller 0.3 are grey. The size of the tiles corresponds to the spearman correlation of pathway footprints between each $\mathrm{KO}$ and the corresponding representative KOs. The colour gradient on the sidebar at the left indicates mean change of naïve marker gene expression (as a measure of phenotypic strength) for the corresponding KO at N24. clusters. Asterisks indicate that the absolute row-wise z-value of the respective mean expression value was above $1.96(p \leq 0.05)$, indicating extreme values within the context of that row. For reference, average naive marker $\log 2 \mathrm{FCs}$ at N24 vs WT are shown in a separate row below (similar to Figure 2a).

805 c, GO enrichment analysis per 'constitutive KO response' cluster. Dot-size scales with log2 enrichment. Adj. p vales are clour coded in shades of red. 

response gene-clusters.

e, As for c, except GO enrichment analysis per 'N24 induced KO response' cluster.

810

\section{Supplementary Figure 6}

812 a, Schematised computational strategy to derive non-overlapping gene-expression footprints

813 for the five signalling pathways.

814 b, Average change in naive marker expression induced by KOs at N24 (y-axis) and their 'LIF-

815 likeness' in 2i (x-axis; correlation of RC9 LIF response with knockout induced response in 2i).

816 Overall correlation across these values is $r=0.69$. The KOs showing the strongest correlations

817 (Tsc2, Ptpn11 and Trim71 KOs) are indicated in red.

818

c, Flow analysis showing delayed Rex1-GFP downregulation in cells starting from cultures in 2i/LIF compared to cells starting from $2 \mathrm{i}$.

820

d, Multiple regression coefficients of pathway activity profiles across all KOs when predicting the differentiation delay phenotype (defined by mean naïve marker gene expression change at N24). Error bars indicate the standard error of the coefficient. exemplary gene expression behaviours. The constitutive and the N24 induced KO response group of genes were used for further analysis.

$826 \mathrm{f}$, Percentages of genes in the N24 induced KO response (N24 induced) or constitutive KO response (constitutive) clusters that significantly change during WT differentiation (FDR $\leq$ 0.05, $\mathrm{HO}:|\mathrm{FC}|<1.5)$. 
832 a, Visualization of connections between pathways (central ellipses) and gene clusters

833 (rectangles) based on multiple regression models. The colour gradient of the clusters indicates

834 enrichment for NAGs. Dashed connections link pathways to clusters that fit time-course

835 validated regression models (see Supplementary Figure 7A and C). Continuous connections

836 are further supported by ChIP or pathway defining gene enrichments (see Supplementary

837 Figure 7E,F). Connections are coloured by source pathway. Unconnected clusters are listed at 838 the right.

839 b, Summary model of the genetic and signaling pathway control of naïve to formative

840 transition. Between the naïve and formative gene regulatory networks, the five signaling

841 pathways largely operate through partially overlapping separable gene clusters.

842

\section{Supplementary Figure 7}

844 a, Heatmap illustrating multiple regression models of pathway activities predicting cluster

845 expression. Only significant (adj. p. $\leq 0.01$ ) interactions are shown. Colour intensity represents

846 the strength of the interaction. Red tiles indicate that higher activity of a certain pathway

847 results in higher expression of the cluster, while blue tiles indicate the opposite.

848 b, Pathway activities (average expression of the 50 specific pathway-defining genes) across a

849 WT differentiation time-course for all 5 pathways. $y$-axis shows average log2FC of pathway

850 defining genes relative to the mean expression throughout the time-course.

851

c, Observed cluster expression change (red) and cluster expression change predicted by

852 regression models (blue) throughout the $32 \mathrm{~h}$ WT differentiation time course for all induced

853 clusters. Predicted cluster expression is calculated using pathway activities over the WT time

854 course and significant (adj. $p \leq 0.01$ ) interactions from the multiple regression models. Graphs 
855 show $\log 2 \mathrm{FC}$ of cluster expression relative to the mean cluster expression across the time

856 course. Red cluster names indicate that cluster-pathway connections were not predicted in

857 Supplementary Figure 7A or validation through $2 \mathrm{~h}$ time-course expression failed (predicted

858 expression did not precede observed expression).

859 d, Similar to Supplementary Figure 7C for constitutive clusters.

860 e, Enrichment from Fisher test of ChIP-targets, NAGs and pathway defining gene sets in

861 induced clusters. Enrichment is indicated by red circles, depletion by blue squares. Size

862 indicates strength of over- or underrepresentation and colour gradient encodes adj. p values.

863 f, Similar to Supplementary Figure 7E for constitutive clusters. 


\section{Cloning}

866 Guide RNAs (gRNAs) were designed using the computational pipeline described below, or by

867 http://crisprscan.org. To clone the gRNAs for ESC KO of the 154 selected genes, we used RecET

868 recombineering to insert the guide RNA protospacer encoding sequence between the U6

869 promoter and the universal sgRNA scaffold in pBR322-U6-cm-ccdB-sgRNA-amp, as described

870 previously (Baker et al, 2016). We generated 331 sgRNA expressing plasmids for 149 genes (2

871 sgRNAs for 131 genes, 3 sgRNAs for 5 genes, 4 sgRNAs for 12 gens and 6 sgRNAs for 1 gene).

872 In brief, the plasmid was linearized at the point of insertion by BstZ17I digestion, purified 873 using the Invitrogen Charge Switch PCR Purification Kit and dissolved in water; 80-mer

874 oligonucleotides containing the protospacer sequence, flanked by $30 \mathrm{bp}$ of homology to the 875 target plasmid were dissolved in water. E. coli GB05 transformed with the pSC101-Prha-ETgA876 tet plasmid ${ }^{63}$, was cultured to OD600 0.5 in deep-well 96 well plates and then induced for 1 877 hour for expression of the ETgA operon with L-rhamnose. Electroporation with $200 \mathrm{ng}$ (0.1 878 pmol) of the linearised vector and 50 pmol of oligonucleotide, using BTX 96 well 879 electroporation plates (MOS96, 2mm gap,) and the BTX ECM630 electroporator with HT-200 880 adapter, was described previously ${ }^{64}$. After one hour of recovery time, 100 microliters (1/10th)

881 of the culture was transferred to a new plate with 900 microliters of LB media plus 100 micrograms/ml ampicillin. After overnight selection the saturated cultures were plated for

883 single colonies on L-agar +100 micrograms/ml ampicillin. A single colony for each construct was picked and the clones were grown under selection in 96 well plates, plasmid DNA was 885 isolated, and Sanger sequenced from both directions using the M13F and M13R primers 886 (flanking the U6-protospacer-sgRNA scaffold cassette, Supplementary Table 6). Sequence 
second colony or repeat cloning for the remaining 15 clones, all the desired constructs were obtained. Alternatively, annealed oligonucleotides (Supplementary Table 6) were cloned into

890 a Bsal site of a gRNA expression vector (Addgene Plasmid number 41824) ${ }^{65}$ and correct

891 insertion was determined by Sanger sequencing with the SP6 primer (Supplementary Table

892 6). For generating rescue cell lines, indicated coding sequences were cloned into a pCAG-

3xFLAG-empty-pgk-hph vector ${ }^{14}$ after PCR amplification and correct insertion was detected by restriction digest and Sanger sequencing with the 3xFlag_seq primer (Supplementary Table 6).

896

897

\section{Cell culture}

898 Diploid (biparental) ES cells were routinely cultured in gelatin coated $25 \mathrm{~cm}^{2}$ flasks in DMEM 899 supplemented with 15\% FCS (batch tested, Biowest), 1x Penicillin-Streptomycin (Sigma),

$900 \quad 0.1 \mathrm{mM}$ NEEA, $1 \mathrm{mM}$ sodium pyruvate, $1 \mathrm{mM}$ L-glutamine, $0.05 \mathrm{mM}$ 2-mercatpoethanol

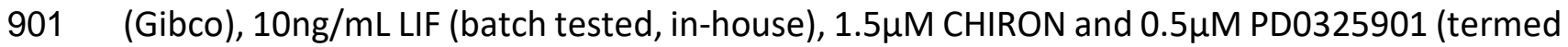
902 ESDMEM-2i) on gelatin coated plates. The basal medium during all differentiation assays and 903 for haploid ES cell culture (N2B27) consisted of a 1:1 mixture of DMEM/F12 and Neurobasal 904 medium supplemented with $1 \times$ B27 (Gibco), 0.5x N2 (homemade), 0.1mM NEAA, 1mM L905 glutamine, 1x Penicillin-Streptomycin and 0.05mM 2-mercaptoethanol. Haploid ES cells were 906 routinely cultured in N2B27 supplemented with $3 \mu \mathrm{M}$ CHIR99201, $1 \mu \mathrm{M}$ PD0325901 and $90710 \mathrm{ng} / \mathrm{ml}$ LIF and regularly sorted based on FSC/SSC parameters by FACS ${ }^{66}$. Haploid Rex1GFPd2-IRES-BSD (hRex1GFPd2, ${ }^{67}$ and haploid Rex1::mKO2-IRES-BSD/pOct4-GFP-IRES-

909 Puro ESCs (generated in this study from a double reporter mouse) were derived from 910 activated oocytes in 2i/LIF medium and used for screens. Biparental diploid ES cells with a GFP

911 driven by the endogenous Rex1 promoter and EF1a driven Cas9 targeted to the Rosa26 locus 
912 (RC9 cells, ${ }^{13}$ served as parental cell line for the KO lines generated in this study. A list of KO

913 cell lines can be found in Supplementary Table 6.

914

Transposon-based saturation screen for key players of the exit from pluripotency

916 The genetrap vectors 5'-PTK-3', TNN, TNP (Horie et al. 2011, Allan Bradley Lab) together with

917 haploid Rex1::GFPd2 ES cells (hRex1GFPd2, Leeb et al. 2014) and haploid Rex1::mKO2-IRES-

918 BSD/pOct4-GFP-IRES-Puro ES cells (hOR, this study) were used in 35 independent experiments

919 to drive the screen to saturation. Mutant pools were generated by electroporation of $10^{7}$

920 haploid ESCs using a GenePulser Xcell ( $270 \mathrm{~V}, 500 \mu \mathrm{F}, \infty \Omega$, Biorad) with $0.5 \mu \mathrm{g}$ genetrap plasmid and $10 \mu \mathrm{g}$ hyperactive transposase (hyPBase) ${ }^{68} .24 \mathrm{~h}$ after electroporation, selection was started for 4 days using $1 \mu \mathrm{g} / \mathrm{ml}$ Puromycin. Thereafter cells were plated at a density of $10^{4}$ cells $/ \mathrm{cm}^{2}$ in N2B27 medium in the absence of LIF or inhibitors to allow differentiation. After 7-10 days in differentiation conditions, GFP-positive cells were sorted and replated at a density of $10^{4}$ cells $/ \mathrm{cm}^{2}$. After culture in N2B27 medium for a further 7-10 days, GFP-positive ESCs were resorted, expanded for $48 \mathrm{~h}$ in $2 \mathrm{i} /$ LIF medium and DNA was isolated using the PureGene kit (Qiagen) according to the manufacturer instructions. Sequencing libraries were prepared using an optimized Splinkerette PCR protocol (Leeb et al. 2014) with a modified and improved set of adapters and primers (see Supplementary Table 6). Adapters were annealed at $50 \mu \mathrm{M}$ each in T4 DNA ligase buffer by incubating at $97.5^{\circ} \mathrm{C}$ for 150 sec followed by a

931 temperature decrease of $0.1^{\circ} \mathrm{C} / 5 \mathrm{sec}$ for 775 cycles. Ready-to-use adapters were stored at $20^{\circ} \mathrm{C}$. Genomic DNA was isolated using the PureGene kit (Qiagen) according to the manufacturers recommendations and quantified on the Qubit 2 fluorometer (Life 

with the following settings: Duty Cycle $-20 \%$, Intensity -5 , Cycles per Burst -200 , Time -60 seconds, Temperature $-4^{\circ}$ to $7^{\circ} \mathrm{C}$. After shearing DNA was isolated with the QIAquick PCR purification kit (Qiagen) and quality assessed using the Agilent Bioanalyzer with the DNA high sensitivity kit (Agilent). End-repair was performed using the NEBNext End Repair Module

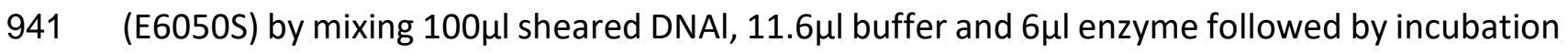
942 at $20^{\circ} \mathrm{C}$ for $60 \mathrm{~min}$. After cleanup with the PureLink PCR Purification Kit (K3100-01), $42 \mu \mathrm{l}$ of the end repaired DNA was A-tailed with the NEBNext dA-Tailing Module (E6053S) with $5 \mu$ l buffer and $3 \mu$ l enzyme at $37^{\circ} \mathrm{C}$ for 60 minutes. Following PureLink purification of to the A-tailed DNA, the annealed adapters were ligated using theNEBNext Quick Ligation (E6056S) kit at $20^{\circ} \mathrm{C}$ for 30min. Products were cleaned up using Ampure XP beads at a 1:1 ratio. Libraries were PCR

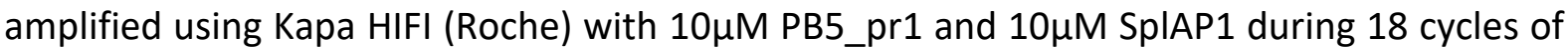
$20 \sec 98^{\circ} \mathrm{C}, 20 \sec 63^{\circ} \mathrm{C}$ and $40 \sec 72^{\circ} \mathrm{C}$ with an initial denaturation at $95^{\circ} \mathrm{C}$ for 2 minutes and a final extension at $72^{\circ} \mathrm{C}$ for $5 \mathrm{~min}$. Samples were cleaned up using Ampure XP beads at a ratio of 0.8. A second round of PCR was conducted using xxul from PCR1 using the Kapa HIFI polymerase with $10 \mu \mathrm{M}$ SpIAP2 and $10 \mu \mathrm{M}$ index primer for 13 cycles at an annealing temperature of $60^{\circ} \mathrm{C}$ with all other settings as in PCR1. Final libraries were quantified using the KAPA SYBR Fast qPCR Mix with primers SYB7 and SYB5 at $10 \mu \mathrm{M}$ each and a final quality control was conducted with the Agilent Bioanalyzer DNA High Sensitivity Assay.I ntegration sites were mapped as in ${ }^{12}$ and described below.

956

Generation of KO ESCs

958 Direct comparison between KOs necessitates a similar culture and passaging history. We 959 therefore established an experimental pipeline to parallelise the generation of multiple KO 
ESC lines per week. $2 \times 10^{5}$ RC9 cells were transfected in a 6-well format with a set of two gRNA

961

962

963

964

965

966

967

968

969

970

971

972

973

974

975

976

977

978

979

980

981

982

983

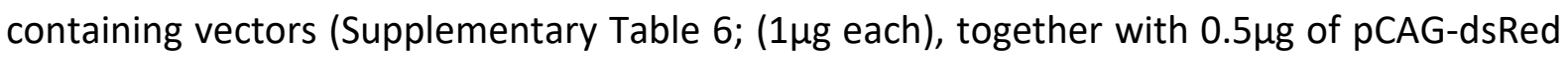

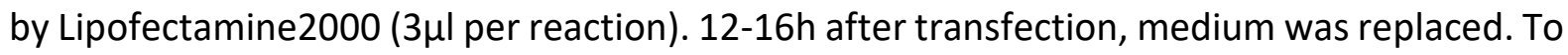
enrich for transfectants, cultures were sorted for GFP/DsRed double positive cells on a BD FACS Aria III 48h after transfection and plated at clonal density in $6 \mathrm{~cm}$ dishes (100 to 800 cells per dish) in ESDMEM2i. Approximately one week after sorting, 48 colonies per gene KO were picked into 96-well plates, trypsinized and split into one expansion-plate and one plate for PCR genotyping after boiling lysis and proteinase $\mathrm{K}$ treatment (see PCR genotyping). Identified KO clones were expanded to 12-well or 6-well plates after 3 days of growth and the remaining plate was frozen in 50\%FCS/10\% DMSO as a backup. Clones were frozen from $12-$ or 6-well plates in freezing medium containing 50\% FCS and $10 \%$ DMSO. Of the attempted 154 gene disruptions, we achieved 115 homozygous knockout cell lines. In 16 cases, only heterozygous clones were obtained (indicating essentiality of these genes), and in 23 cases we were unable to recover gene disruptions, despite multiple attempts (Supplementary Table 6).

\section{PCR genotyping}

Crude DNA lysates were generated by pelleting half of a picked colony in a PCR plate at 500g at $4^{\circ} \mathrm{C}$, followed by two washes with PBS. $25 \mu$ l of water were added and pellets were boiled for $5 \mathrm{~min}$ at $95^{\circ} \mathrm{C}$. After cooling, Proteinase $\mathrm{K}$ was added at a final concentration of $3 \mu \mathrm{g} / \mu \mathrm{l}$ and incubated at $65^{\circ} \mathrm{C}$ for $1 \mathrm{~h}$ followed by inactivation at $95^{\circ} \mathrm{C}$ for $10 \mathrm{~min}$. Successful KO generation was confirmed by PCR, employing a three-primer strategy. Specific reverse primers for the deletion event and a possible wildtype allele were used in combination with a common forward primer (Supplementary Table 6). Cell lines with indels caused by a NHEJ event around 
the exonic gRNA1 target site, were detected by Sanger sequencing of the "wildtype"-band.

Verification of the KO-events was performed by the same PCR strategy on DNA, isolated using the PureGene DNA isolation kit (Qiagen) according to the manufacturer recommendations. according to the manufacturers recommendations for cycling and a standard annealing temperature of $55^{\circ} \mathrm{C}$ and $35-40$ cycles.

990

991

\section{Immunoblotting}

Whole cell fractions were isolated using RIPA buffer and protein concentrations were

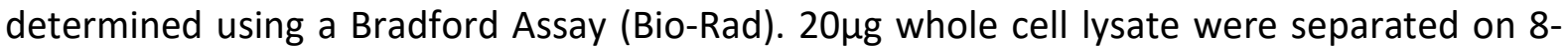
$12 \%$ SDS-PAGE gels (depending on the molecular weight of the target proteins), and subsequently blotted on $0.2 \mu \mathrm{m}$ nitrocellulose membranes (Amersham). Membranes were for $1 \mathrm{~h}$. Primary antibodies were incubated overnight at $4^{\circ} \mathrm{C}$, at a dilution of $1 / 1000$ with the

Tubulin 1/5000, Anti-Smg5 1/200, Smg7 1/2000. Secondary goat anti-mouse IgG HRP

1000 (1:10000) or goat anti-rabbit IgG HRP (1:15000) were incubated at RT for $1 \mathrm{~h}$ in blocking solution. Antibody binding was detected using the ECL select detection kit (Amersham). A list of antibodies can be found in Supplementary Table 6.

\section{Parallel Differentiation}

1005 ES Cells were differentiated in seven batches, always including WT controls, control KO ESCs 1006 (no phenotype expected) and a range of ESCs with expected weak to strong phenotypes. 1007 Where applicable two independent KO clones were used, other KO ESCs were cultured in 
parallel replicates before intiation of differentiation. $24 \mathrm{~h}$ before starting the differentiation assay, medium was changed from ESDMEM2i to N2B27 based 2i/LIF medium. Cells were then trypsinized, counted and plated in $2 \mathrm{i}$ (without LIF) at a density of $10^{4}$ cells $/ \mathrm{cm}^{2}$ in two replicate wells of a 6-well plate for differentiation, one 6-well as undifferentiated control and 2 wells of a 12-well plate (undifferentiated control and differentiated sample) to monitor differentiation by FACS. $12 \mathrm{~h}$ after plating, cells were washed twice with PBS and medium was changed to unsupplemented N2B27. For the undifferentiated controls medium was replaced with fresh $2 \mathrm{i}$. $24 \mathrm{~h}$ later, at N24, cultures were harvested in RLT buffer and stored at $-80^{\circ} \mathrm{C}$ before isolation of RNA using the RNeasy Mini Kit (Qiagen). mRNA was isolated from 1 ug total RNA by poly-dT enrichment using the NEBNext Polya, mRNA Magnetic Isolation Module according to the manufacturer's instructions. Samples were eluted in $15 \mathrm{ul} 2 \mathrm{x}$ first strand cDNA synthesis buffer (NEBnext, NEB). After chemical fragmentation by incubating for 15 min at $94^{\circ} \mathrm{C}$ the sample was directly subjected to the workflow for strand specific RNA-Seq library preparation (Ultra Directional RNA Library Prep, NEB). For ligation custom adapters were (Supplementary Table 6). After ligation, adapters were depleted by an XP bead purification

1023 (Beckman Coulter) adding beads in a ratio of 1:1, followed by an index PCR (15 cycles) using Illumina compatible index primer. After double XP beads purifications (with beads added in a ratio 1:1) libraries were quantified on a Fragment Analyzer run with a NGS Assay Kit (Agilent) and loaded on a HiSeq 3000 flowcell with 50 cycles single end sequencing by pooling the

1027 samples based on molarity aiming for 30 mio. reads per sample.

1028

\section{$2 \mathrm{~h}$ resolved differentiation timecourse}

Medium was changed to N2B27-2iLIF 24h before the assay. Cells were trypsinized, counted and plated at a density of $10^{4}$ cells $/ \mathrm{cm}^{2}$ in 6 -well plates in $2 \mathrm{i}$ (without LIF). $12 \mathrm{~h}$ later medium 
was changed to unsupplemented N2B27 (or 2i for the undifferentiated controls) and cells were harvested in buffer RLT every 2 hours for the next 32h (N32). RNA was isolated using the RNeasy Mini Kit (Qiagen). All libraries were generated from 500ng RNA using the TruSeq Stranded mRNA Library Prep Kit (Illumina) and analysed on a HiSeq4000, Paired End 75bp.

\section{LIF signature}

$10381 \times 10^{4 c}$ ells $/ \mathrm{cm}^{2}$ were plated in N2B27-2i and N2B27-2iLIF and grown for $24 \mathrm{~h}$ after which RNA was isolated using the RNeasy Mini Kit (Qiagen). Libraries were made from $1 \mu \mathrm{g}$ high quality RNA using the Quantseq 3' mRNA-Seq Library Prep Kit FWD for Illumina (Lexogen) according to the manufacturer's instructions. Libraries were quantified using qPCR according to the

1042 Quantseq Library Prep Kit and with the included primers, pooled and quality checked using an Agilent Bioanalyzer DNA High Sensitivity Assay (Agilent). Multiplexes were sequenced on a HiSeq4000, Single End 50bp.

\section{RNAi experiments}

1047 For RNAi FlexiTube siRNAs against KIf2 were used with AllStars Negative Control siRNAs

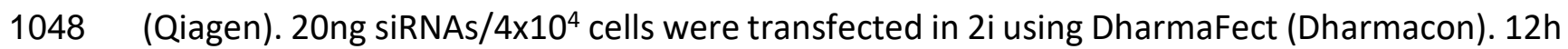
1049 later medium was changed to N2B27 after two PBS washes. At N24 and N32 Rex1GFPd2 expression was determined by flowcytometry (see Flowcytometry Analysis) and cells were

1051 harvested in RLT buffer and isolated with the RNeasy mini kit (Qiagen) according to the manufacturer's recommendations. cDNA was transcribed using the SensiFAST cDNA Synthesis Kit (Bioline). Naïve and primed marker expression was determined by qPCR using the Sensifast SYBR No Rox-Kit (Bioline). Primers are listed in Supplementary Table 6. 
1057 Cells were dissociated in $0.25 \%$ trypsin/EDTA and trypsin was neutralized with DMEM

1058 supplemented with 15\% FCS. After passing through a $40 \mu \mathrm{m}$ mesh, Rex1-reporter activity was

1059 measured using a BD Fortessa machine. High-throughput-measurements were performed in

1060 96-well plates using the HTS unit of the BD Fortessa. Data were analyzed using FlowJo 1061 software. 
Data Analysis

\section{Transposon Mutagenesis Screen in Haploid Murine Embryonic Stem Cells}

Integration mapping was performed as previously described ${ }^{12}$. Most gene-trap insertions

were found in intronic sequences, consistent with the relative length distributions of genomic

features. However, after normalising to available TTAA sites per region, TTAA sites in 5'UTRs

1067

and promoters (<-500bp) were overrepresented, whereas TTAA sites in introns were

1068

relatively depleted. 7760 independent integrations passing cut-off criteria were mapped to

1069

3469 genes. Of those, 232 Genes (2474 independent integration sites) were hit in five or more

1070 independent screens, indicating that those are integrations causative for the detected

1071

differentiation defect. To complement the candidate list and correct for gene specific biases

1072

we further developed a strategy to assign statistical strength to candidate genes by assessing

1073

the number of integrations per gene in relation to available TTAA transposon integration sites

1074

We utilized the number of different TTAA-sites within each gene to calculate the probability

1075

of having integrations in $k$ or more different locations by chance. All TTAA sites in mm10 were

1076 assessed using bowtie and the analysis was restricted to genes classified as protein coding,

1077 long non-coding RNAs and micro RNAs. We computed the probability that a gene is hit $k$ times

1078 given that it contains $n$ TTAA transposon integration sites assuming a binomial distribution

1079 (while $k$ is the observed number of integrations).

1080

$$
P[X \geq i]=\sum_{i=k}^{n} P[X=i]=\sum_{i=k}^{n} B\left(i \mid p_{0}, n\right)=\sum_{i=k}^{n}\left(\begin{array}{c}
n \\
i
\end{array}\right) p_{0}^{i}\left(1-p_{0}\right)^{n-i}
$$

1081

po was then calculated by $\frac{\text { All hit TTAA-sites in genes }}{\text { All TTAA-sites in genes }}$ 
We applied this test to genes with at least 26 TTAA sites, as genes with fewer TTAA sites would

be significant $(p$-value $<0.05)$ with a single integration. Candidate gene lists between both

\section{Guide RNA design criteria}

1087

To automate the design of sgRNAs as far as possible we used the R package CRISPRseek (Zhu et al., 2014) to score the efficiency and likely off-target binding of candidate sgRNA sequences. For a selected gene the algorithm proceeds as follows:

1090

1. Retrieve the sequence of the first protein coding exon

2. Sequence has to be at least 50 bases long

3. Find sgRNAs within the sequence and score them.

4. If no appropriate sgRNA could be found, move to next exon

5. Still in the first third of the coding sequence?

1098 A minimal efficiency of 0.2 and an off-target score $<100$ was required.

1100 The surrounding regions of the sgRNAs (min distance 20bp) were used as input for PRIMER3 1101 primer predictions. Primers were designed to have a length of 18-27 bases with an optimum 1102 of 20 and to have melting temperatures between $57^{\circ} \mathrm{C}$ and $63^{\circ} \mathrm{C}$ with an optimum of $60^{\circ} \mathrm{C}$. All 
primers were checked for off-target binding sites by using Blastn. Blastn was called with the

1104 parameters:

1105 -task megablast -use_index false -word_size 7 -perc_identity 65 -evalue 30000 -

1106 max_target_seqs 50 -num_threads 4 -outfmt 7.

\section{RNA quantification, RNAseq}

1108 Quality control was performed using fastQC (version 0.11.5). Transcripts were mapped to the

1109 mm10 mouse reference genome and counts determined using STAR (version 2.5.3). RPKM

1110 values were calculated using DeSEQ2 (version 1.20.0).

\section{RNA quantification, QuantSeq}

1112 Transcripts from RC9 cells in 2i and 2i/LIF medium were measured using QuantSeq (Lexogen)

1113 and quantified as described. Reads were trimmed with bbduk (version 35.92). Quality control

1114 was performed using fastQC (version 0.11.5). Transcripts were mapped to the mm10 mouse

1115 reference genome using STAR (version 2.5.3). After indexing with samtools (version 1.3) reads

1116 in genes were counted using HTSeq-count (version 0.6.0). Counts were subjected to

1117 differential expression analysis.

\section{Differential expression analysis}

1119 Differential expression analysis was carried out using limma (version 3.30.13), after

1120 transforming transcript counts using the included voom function. We then fitted a linear

1121 model to sample measurements from each combination of knockouts (including RC9 control)

1122 and conditions ( $2 \mathrm{i}$ and N24). RNAseq batches were included in the model as a confounding

1123 factor. Contrasts were fitted to determine the expression differences between a) each 
1124 knockout sample in $2 \mathrm{i}$ and the RC9 control, b) each knockout sample in N24 and the RC9

1125 control, and c) the difference between the log-fold-changes calculated in a) and b)

1126 (interaction effect). We also fitted a contrast to determine changes during normal

1127 differentiation, i.e. the differential expression between RC9N24 versus RC92i. All fitted

1128 contrasts were tested for differential expression using moderated t-statistics through the

1129 limma functions eBayes (HO of no change) or treat (HO interval [- $\log 2(1.5), \log 2(1.5)]) . p-$

1130 Values were corrected for multiple testing using the Benjamini Hochberg method (FDR). For

1131 subsequent analyses the more stringent $p$-values and FDR values as calculated by treat were

1132 used (unless noted otherwise). Differential expression analysis of 2i/LIF vs. 2i RC9 measurements was carried out separately, but in an identical fashion.

\section{GO enrichment analysis}

1135 Mouse gene GO annotations were extracted from the R package org.Mm.eg.db (version 1136 3.4.0). GO terms that included between 5 and 500 genes were selected for further analysis.

1137 For each gene list of interest, significance of functional enrichment of GO terms compared to 1138 the background list was determined using Fisher's exact tests. The background was restricted

1139 to genes whose transcripts were detected at a median count above 5. To reduce redundancy 1140 of functional categories, we clustered GO terms that differed in 5 or fewer genes of interest

1141 (using the R base function hclust on the L1-distance of the binary membership matrix). The 1142 smallest GO term by total annotations was selected independently from Fisher test results as 1143 the primary, i.e. most specific term to represent each cluster. We then carried out correction

1144 for multiple hypothesis testing using the Benjamini-Hochberg method on all primary GO 1145 terms to obtain adjusted $p$-values. 
1147 To quantify the similarity of each gene's differential expression (DE) pattern across KOs at N24

1148 with the DE pattern of seven core pluripotency markers (Nanog, Esrrb, Tbx3, Tfcp2l1, Klf4,

1149 Prdm14 and Zfp42), multiple regression analysis was performed. I.e., each gene's DE pattern

1150 was modelled as a linear combination of marker DE patterns, quantifying the strength of

1151 association as their shared variance (multiple- $R^{2}$ ). NAGs were defined as having a naïve

1152 marker $\mathrm{R}^{2}$ of $\geq 0.7$.

\section{Cluster analysis (constitutive \& N24 induced)}

1154 Based on the responses to the knockout conditions (excluding Suz12, Eed \& Csnk1a1 KOs) in

1155 both $\mathrm{N} 24$ and $2 \mathrm{i}$ and their interactions with the RC9 control, we defined two main categories

1156 of responsive genes: (1) the constitutive knockout response: genes that were significantly

1157 changed (adj. $\mathrm{p}-\leq 0.05$ ) in the same direction in both $\mathrm{N} 24$ and $2 \mathrm{i}$, in at least one knockout condition. Genes from the N24 induced KO response category were excluded. (2) N24 induced knockout response: genes that were significantly changed in both N24 and the knockout:RC9 interaction term, in at least one knockout condition. To determine whether genes in the N24

1161 induced knockout response subset were overall more correlated with naive pluripotency markers, we checked the distribution of naive marker multiple-R2 values in the 'N24 induced knockout response' set compared to both the background distribution and genes classified as part of the constitutive KO response.

1166 To determine if distinct functional clusters of genes that may be activated in all, single, or sub-

1167 groups of knockouts, we clustered genes in either of the categories (constitutive and N24 1168 induced response) based on their $\mathrm{KO}^{\mathrm{N} 2}$ vs. RC9 ${ }^{\mathrm{N} 24}$ log-fold-changes, using the R base package 
1169 hclust with the "Ward.D2" method. We determined the total within-cluster variance at

1170 different numbers of clusters. The number of clusters was chosen such that the model

1171 improved strongly by increasing the number of clusters up to that value, but only marginal

1172 gains result from increasing it further. Thereby 12 gene clusters in the constitutive response

1173 and 12 clusters in the induced response were identified. GO enrichment analysis was carried

1174 out as described above for member genes of each cluster

\section{Differentiation pathway correlation analysis}

1176 We defined downstream responses of key differentiation pathways using the most specific

1177 responses to the knockouts of their upstream regulators: Tsc2 (mTOR), Ptpn11 \& Fgfr1

1178 (Fgf/Erk signalling), Tcf7l1 (Wnt signalling), and Rbpj (Notch signalling). In addition, LIF-specific

1179 changes were defined as the response of genes in RC9 cells in the $2 \mathrm{i} /$ LIF compared to the $2 \mathrm{i}$

1180 condition. To ensure specificity, we required that the log-fold-change of each response gene

1181 for a given regulator (in N2B27 medium) exceeded that observed in the remaining conditions

1182 by a factor of two. In the case of Ptpn11 \& Fgfr1, this criterion had to be met by the lower of

1183 the two log-fold-changes for each gene. Additionally, the genes were required to satisfy a

1184 FPKM (CPM in case of LIF) cut-off of at least 1 in either the KO or the WT. From all remaining,

1185 significantly expressed genes (FDR $\leq 0.05$ ) we then selected, by absolute log-fold-change in

1186 descending order, the top 50 most specific genes for each pathway (Supplementary Figure

1187 6a).

1189 Pathway activity was determined across all other knockouts at N24. For each pathway, the

1190 log-fold-changes of the 50 pathway-specific response genes from the associated regulator

1191 knockout (KO ${ }^{\mathrm{N} 24}$ vs. RC9 ${ }^{\mathrm{N} 24}$ ) were selected. We then correlated these log-fold-changes to the 
log-fold-changes of the same genes in all other knockouts. A positive correlation indicates

1193 that the pathway is disrupted in a manner similar to knocking out its upstream regulator, whereas a negative correlation indicates the opposite. In the case of LIF-specific genes, a positive correlation indicates an effect similar to that of adding LIF. The analysis was repeated for all $2 \mathrm{i}$ experiments (using the same lists of specific genes as in N24). P-values of correlation values were calculated based on Fisher's Z transform. P-values were then corrected for multiplicity using the Bonferroni method.

1200 To determine how predictive the calculated correlations were of the differentiation phenotype (mean naive marker logFC vs. RC9 in N2B27), we carried out multiple linear regression and extracted the coefficients of the model to establish the relative contribution of each pathway signature to naïve marker deregulation.

\section{Quantification of differentiation delay in knockouts}

1205 Raw RNA sequencing data was aligned to the mm10 genome and read counts were obtained using the STAR aligner (version2.5.2b). Gene expression for the following biotypes was quantified: protein_coding, misc_RNA, miRNA, scaRNA, scRNA, snoRNA, snRNA, sRNA. Read counts were normalized using DESeq2 (version 1.22.2) and subsequently the log2fc between all samples and the mean expression of the $2 \mathrm{i}$ samples was calculated. This resulted in expression profiles for each gene over 32 hours of differentiation.

1212 Gaussian process regression was used to smoothen the profiles of each gene (R package tgp 1213 version 2.4-14). The expression profiles of 73 knockouts at N24 were mapped on the 1214 differentiation axis from the time course to quantify differentiation delays per KO, i.e. we 
aimed to quantify how many hours the KO expression pattern is 'behind' the expected differentiation in WT cells. This 'mapping' to the time axis was done first computing the logFCs between $\mathrm{N} 24$ versus $2 \mathrm{i}$ for each $\mathrm{KO}$. The resulting logFC profiles were compared to the logFCs at each time point during the WT differentiation. We assumed that the time point at which the difference between the WT profile and the KO profile is minimized best reflects the molecular differentiation state of the respective KO.

We used the Euclidean distance for this purpose. To make distances better interpretable between the knockouts the distances were set to:

1223

where the maximum and minimum distances refer to the respective maximum and minimum Euclidean distances of the respective KO across all time points (i.e. the worst and the best matching time point). Thus, the best matching time point will get a distance score of 0 .

1230 In order to further increase the time resolution, additional time points were imputed via linear interpolation. Here we interpolated expression profiles every 15 minutes between the two neighbouring time points of the time point with the smallest Euclidean distance. Timing of used to quantify the differentiation delay.

\section{Prediction of cluster expression by pathway activity}




$$
\text { directed } F C(K O)=F C\left(\frac{K O}{W T}\right) *\left\{\begin{array}{c}
L I F\left\{\begin{array}{c}
+1 \text { same direction as in } \frac{L I F}{2 i} \text { comparison } \\
-1 \text { opposite direction as in } \frac{L I F}{2 i} \text { comparison }
\end{array}\right. \\
T s c 2, P 11 F 1, T c f 7 l 1, R \operatorname{lopj}\left\{\begin{array}{c}
+1 \text { same direction as in } \frac{\text { rep. } K O}{W T} \text { comparison } \\
-1 \text { opposite direction as in } \frac{\text { rep. } K O}{W T} \text { comparison }
\end{array}\right.
\end{array}\right.
$$

$$
\text { directed avg. } \log F C_{K O}=\frac{\sum_{n}^{P W} \text { specific marker genes directed } F C(K O)_{n}}{\sum_{n}^{P W} \text { specific marker genes directed } F C(\text { rep. } K O)_{n}}
$$

$$
\text { pathway activity }_{K O}=\text { directed avg. } \log F C_{K O} *\left\{\begin{array}{c}
1 \text { for } L I F, T s c 2 \\
-1 \text { for } P 11 F 1, T c f 7 l 1, R b p j
\end{array}\right.
$$

1243 The combinations of pathway activities and average cluster expression changes over all KOs were used to build a multiple regression model to predict the expression changes in each

1245 cluster by the five pathway activities. Connections between pathway activities and cluster 1246 expression changes not making a significance cut-off (padj $<=0.01$ ) of the multiple regression 1247 models were excluded from each model. The remaining model was tested in the time course 1248 data. Connections from clusters where prediction of expression changes based on pathway activities did follow or contradict the observed expression changes in the time course were excluded.

\section{Embryo comparison}

1253 Sequencing data were obtained from the European Nucleotide Archive ${ }^{69}$ from single-cell mouse embryo profiling studies ${ }^{10,70}$. Human capacitation dataset was downloaded from

1255 Gene Expression Omnibus website (GSE123055). The macaque FKPM expression dataset was provided by ${ }^{33}$. Orthologues genes mapping in 1-to-1 fashion were used during comparative analysis. Alignments to gene loci were quantified with htseq-count ${ }^{71}$ based on annotation 
from Ensembl $87^{69}$. Principal component and cluster analysis were based on log2 FPKM values computed with the Bioconductor package DESeq ${ }^{72}$, custom scripts and FactoRmineR packages ${ }^{73}$. High variable genes were calculated by fitting a non-linear regression curve between average log2 FPKM and the square of coefficient of variation. Specific thresholds were applied along the $x$-axis (log2FPKM) and y-axis (coefficient of variation) to identify the most variable genes. Fractional identity between the bulk RNAseq $(2 \mathrm{i}, \mathrm{N} 24)$ and the mouse, macaque embryo or human capacitation dataset were computed using $\mathrm{R}$ package DeconRNASeq ${ }^{74}$.

\section{GENEES (Genetic Network Explorer for Émbryonic Stem Cell Differentiation)}

1267 To both visualize and interrogate gene expression analysis of all KO ES cells we have set up 1268 the online tool GENEES (Genetic Network Exploration of Embryonic Stem Cell Differentiation).

1269 It is accessible at [http://shiny.cecad.uni-koeln.de:3838/stemcell_network/; User: stemcells;

$1270 \mathrm{Pw}$ : S3m7KmlfHr9]. The app integrates multiple visualisations and summary statistics to make

1271 all information, from the gene level to network modules / pathways / GO terms, directly 1272 available for exploratory analysis. The app has 4 different tabs for inspection of single genes, 1273 predefined genesets, pre-computed clusters and custom genesets. Single genes can be 1274 inspected in the "Genes tab", while predefined genesets are available for selection under the 1275 "Genesets" tab. The previously described constitutive clusters and InteractionN2 clusters can 1276 be further inspected in the "Pre-computed clusters" tab. In the "Select custom geneset" tab, 1277 a set of genes of interest subjected to analysis. 


\section{References}

1278

1279

1280

1281

1282

1283

1284

1285

1286

1287

1288

1289

1290

1291

1292

1293

1294

1295

1296

1297

1298

1299

1300

1301

1302

1303

1304

1305

1306

1307

1308

1309

1310

1311

1. Smith, A. Formative pluripotency: the executive phase in a developmental continuum. Development 144, 365-373 (2017).

2. Kalkan, T. et al. Tracking the embryonic stem cell transition from ground state pluripotency. Development 144, 1221-1234 (2017).

3. Buecker, C. et al. Reorganization of Enhancer Patterns in Transition from Naive to Primed Pluripotency. Cell Stem Cell 14, 838-853 (2014).

4. Dunn, S.J., Martello, G., Yordanov, B., Emmott, S. \& Smith, A.G. Defining an essential transcription factor program for naive pluripotency. Science 344, 1156-1160 (2014).

5. Niwa, H. The principles that govern transcription factor network functions in stem cells. Development 145, dev157420 (2018).

6. Chen, X. et al. Integration of external signaling pathways with the core transcriptional network in embryonic stem cells. Cell 133, 1106 - 1117 (2008).

7. Ying, Q.L. et al. The ground state of embryonic stem cell self-renewal. Nature 453, 519-523 (2008).

8. Boroviak, T., Loos, R., Bertone, P., Smith, A. \& Nichols, J. The ability of inner-cell-mass cells to self-renew as embryonic stem cells is acquired following epiblast specification. Nature cell biology 16, 516-528 (2014).

9. Acampora, D. et al. Loss of the Otx2-Binding Site in the Nanog Promoter Affects the Integrity of Embryonic Stem Cell Subtypes and Specification of Inner Cell Mass-Derived Epiblast. Cell reports 15, 2651-2664 (2016).

10. Mohammed, H. et al. Single-Cell Landscape of Transcriptional Heterogeneity and Cell Fate Decisions during Mouse Early Gastrulation. Cell reports 20, 1215-1228 (2017).

11. Leeb, M. \& Wutz, A. Derivation of haploid embryonic stem cells from mouse embryos.

Nature 479, 131-134 (2011).

12. Leeb, M., Dietmann, S., Paramor, M., Niwa, H. \& Smith, A. Genetic exploration of the exit from self-renewal using haploid embryonic stem cells. Cell Stem Cell 14, 385-393 (2014).

13. Li, M. et al. Genome-wide CRISPR-KO Screen Uncovers mTORC1-Mediated Gsk3 Regulation in Naive Pluripotency Maintenance and Dissolution. Cell reports 24, 489-502 (2018).

14. Betschinger, J. et al. Exit from Pluripotency Is Gated by Intracellular Redistribution of the bHLH Transcription Factor Tfe3. Cell 153, 335-347 (2013).

15. Guo, G., Huang, Y., Humphreys, P., Wang, X. \& Smith, A. A PiggyBac-based recessive screening method to identify pluripotency regulators. PloS one 6, e18189 (2011).

16. Mulas, C., Kalkan, T. \& Smith, A. NODAL Secures Pluripotency upon Embryonic Stem Cell Progression from the Ground State. Stem cell reports 9, 77-91 (2017). 
17. Kokubu, C. \& Takeda, J. When half is better than the whole: advances in haploid embryonic stem cell technology. Cell Stem Cell 14 (2014).

18. Elling, U. et al. Forward and reverse genetics through derivation of haploid mouse embryonic stem cells. Cell Stem Cell 9, 563-574 (2011).

19. Kalkan, T. \& Smith, A. Mapping the route from naive pluripotency to lineage specification. Philosophical Transactions of the Royal Society of London B: Biological Sciences 369 (2014).

20. Ye, J. \& Blelloch, R. Regulation of pluripotency by RNA binding proteins. Cell Stem Cell 15, 271-280 (2014).

21. Kalkan, T. et al. Complementary Activity of ETV5, RBPJ, and TCF3 Drives Formative Transition from Naive Pluripotency. Cell Stem Cell 24, 785-801.e787 (2019).

22. Ursu, A. et al. Epiblastin A Induces Reprogramming of Epiblast Stem Cells Into Embryonic Stem Cells by Inhibition of Casein Kinase 1. Cell Chem Biol 23, 494-507 (2016).

23. Martello, G. \& Smith, A. The nature of embryonic stem cells. Annual review of cell and developmental biology 30, 647-675 (2014).

24. Qiu, D. et al. KIf2 and Tfcp2l1, Two Wnt/B-Catenin Targets, Act Synergistically to Induce and Maintain Naive Pluripotency. Stem cell reports 5, 314-322 (2015).

25. Hall, J. et al. Oct4 and LIF/Stat3 additively induce Kruppel factors to sustain embryonic stem cell self-renewal. Cell Stem Cell 5, 597-609 (2009).

26. Yeo, J.C. et al. KIf2 is an essential factor that sustains ground state pluripotency. Cell Stem Cell 14, 864-872 (2014).

27. Martello, G. et al. Esrrb is a pivotal target of the Gsk3/Tcf3 axis regulating embryonic stem cell self-renewal. Cell Stem Cell 11, 491-504 (2012).

28. Moreira, S. et al. Endogenous BiolD elucidates TCF7L1 interactome modulation upon GSK-3 inhibition in mouse ESCs. bioRxiv, 431023 (2018).

29. Wray, J. et al. Inhibition of glycogen synthase kinase-3 alleviates Tcf3 repression of the pluripotency network and increases embryonic stem cell resistance to differentiation. Nature cell biology 13, 838-845 (2011).

30. Shy, B.R. et al. Regulation of Tcf7I1 DNA binding and protein stability as principal mechanisms of Wnt/ $\beta$-catenin signaling. Cell reports 4, 1-9 (2013).

31. Mayer, D. et al. Zfp281 orchestrates interconversion of pluripotent states by engaging Ehmt1 and Zic2. The EMBO journal 39, e102591 (2020).

32. Rossant, J. \& Tam, P.P.L. Exploring early human embryo development. Science 360, 10751076 (2018).

33. Nakamura, T. et al. A developmental coordinate of pluripotency among mice, monkeys and humans. Nature 537, 57-62 (2016). 
34. Rostovskaya, M., Stirparo, G.G. \& Smith, A. Capacitation of human naïve pluripotent stem cells for multi-lineage differentiation. Development 146, dev172916 (2019).

35. Artus, J., Kang, M., Cohen-Tannoudji, M. \& Hadjantonakis, A.-K. PDGF signaling is required for primitive endoderm cell survival in the inner cell mass of the mouse blastocyst. Stem cells (Dayton, Ohio) 31, 1932-1941 (2013).

36. Boroviak, T. et al. Single cell transcriptome analysis of human, marmoset and mouse embryos reveals common and divergent features of preimplantation development. Development 145 (2018).

37. Plusa, B., Piliszek, A., Frankenberg, S., Artus, J. \& Hadjantonakis, A.-K. Distinct sequential cell behaviours direct primitive endoderm formation in the mouse blastocyst. Development 135, 3081-3091 (2008).

38. Smith, A.G. et al. Inhibition of pluripotential embryonic stem cell differentiation by purified polypeptides. Nature 336, 688-690 (1988).

39. Watanabe, S. et al. Activation of Akt signaling is sufficient to maintain pluripotency in mouse and primate embryonic stem cells. Oncogene 25, 2697-2707 (2006).

40. Kunath, T. et al. FGF stimulation of the Erk1/2 signalling cascade triggers transition of pluripotent embryonic stem cells from self-renewal to lineage commitment. Development 134, 2895-2902 (2007).

41. Lowell, S., Benchoua, A., Heavey, B. \& Smith, A.G. Notch promotes neural lineage entry by pluripotent embryonic stem cells. PLoS biology 4, e121 (2006).

42. Molotkov, A., Mazot, P., Brewer, J.R., Cinalli, R.M. \& Soriano, P. Distinct Requirements for FGFR1 and FGFR2 in Primitive Endoderm Development and Exit from Pluripotency. Developmental cell 41, 511-526 e514 (2017).

43. Lake, R.J., Tsai, P.-F., Choi, I., Won, K.-J. \& Fan, H.-Y. RBPJ, the Major Transcriptional Effector of Notch Signaling, Remains Associated with Chromatin throughout Mitosis, Suggesting a Role in Mitotic Bookmarking. PLoS genetics 10, e1004204 (2014).

44. Ohtsuka, S., Nakai-Futatsugi, Y. \& Niwa, H. LIF signal in mouse embryonic stem cells. JAKSTAT 4, e1086520-e1086520 (2015).

45. Villegas, F. et al. Lysosomal Signaling Licenses Embryonic Stem Cell Differentiation via Inactivation of Tfe3. Cell Stem Cell 24, 257-270 e258 (2019).

46. Niwa, H., Ogawa, K., Shimosato, D. \& Adachi, K. A parallel circuit of LIF signalling pathways maintains pluripotency of mouse ES cells. Nature 460, 118-122 (2009).

47. Hoffman, J.A., Wu, C.I. \& Merrill, B.J. Tcf7l1 prepares epiblast cells in the gastrulating mouse embryo for lineage specification. Development 140, 1665-1675 (2013).

48. $\mathrm{Xu}, \mathrm{T}$. et al. RBPJ/CBF1 interacts with L3MBTL3/MBT1 to promote repression of Notch signaling via histone demethylase KDM1A/LSD1. The EMBO journal 36, 3232-3249 (2017).

49. Amano, T. et al. Zscan4 restores the developmental potency of embryonic stem cells. Nature Communications 4, 1966 (2013). 
50. Macfarlan, T.S. et al. Embryonic stem cell potency fluctuates with endogenous retrovirus activity. Nature 487, 57-63 (2012).

51. Chen, E.Y. et al. Enrichr: interactive and collaborative HTML5 gene list enrichment analysis tool. BMC Bioinformatics 14, 128 (2013).

52. Quenneville, S. et al. In Embryonic Stem Cells, ZFP57/KAP1 Recognize a Methylated Hexanucleotide to Affect Chromatin and DNA Methylation of Imprinting Control Regions. Molecular Cell 44, 361-372 (2011).

53. Takahashi, K. \& Yamanaka, S. Induction of pluripotent stem cells from mouse embryonic and adult fibroblast cultures by defined factors. Cell 126, 663-676 (2006).

54. Xie, H., Ye, M., Feng, R. \& Graf, T. Stepwise Reprogramming of B Cells into Macrophages. Cell 117, 663-676 (2004).

55. Lassar, A.B., Paterson, B.M. \& Weintraub, H. Transfection of a DNA locus that mediates the conversion of 10T1 2 fibroblasts to myoblasts. Cell 47, 649-656 (1986).

56. Ramos-lbeas, P. et al. Pluripotency and $\mathrm{X}$ chromosome dynamics revealed in pig pregastrulating embryos by single cell analysis. Nature Communications 10, 500 (2019).

57. Bredenkamp, N. et al. Efficient RNA-mediated reprogramming of human somatic cells to naïve pluripotency facilitated by tankyrase inhibition. bioRxiv, 636670 (2019).

58. Chambers, I. \& Tomlinson, S.R. The transcriptional foundation of pluripotency. Development 136, 2311-2322 (2009).

59. Nichols, J. \& Smith, A. Pluripotency in the embryo and in culture. Cold Spring Harb Perspect Biol 4, a008128 (2012).

60. Boroviak, T. \& Nichols, J. The birth of embryonic pluripotency. Philosophical Transactions of the Royal Society B: Biological Sciences 369 (2014).

61. Boroviak, T. \& Nichols, J. Primate embryogenesis predicts the hallmarks of human naïve pluripotency. Development (Cambridge, England) 144, 175-186 (2017).

62. Rossant, J. \& Tam, P.P.L. New Insights into Early Human Development: Lessons for Stem Cell Derivation and Differentiation. Cell Stem Cell 20, 18-28 (2017).

63. Baker, O. et al. RAC-tagging: Recombineering And Cas9-assisted targeting for protein tagging and conditional analyses. Sci Rep 6, 25529 (2016).

64. Sarov, M. et al. A genome-scale resource for in vivo tag-based protein function exploration in C. elegans. Cell 150, 855-866 (2012).

65. Mali, P. et al. RNA-Guided Human Genome Engineering via Cas9. Science 339, 823-826 (2013).

66. Leeb, M., Perry, A.C.F. \& Wutz, A. Establishment and Use of Mouse Haploid ES Cells, in Current Protocols in Mouse Biology (John Wiley \& Sons, Inc., 2015). 
67. Leeb, M. et al. Germline potential of parthenogenetic haploid mouse embryonic stem cells. Development 139, 3301-3305 (2012).

68. Yusa, K., Zhou, L., Li, M.A., Bradley, A. \& Craig, N.L. A hyperactive piggyBac transposase for mammalian applications. Proceedings of the National Academy of Sciences of the United States of America 108, 1531-1536 (2011).

69. Toribio, A.L. et al. European Nucleotide Archive in 2016. Nucleic Acids Res 45, D32-D36 (2017).

1427 70. Deng, Q., Ramsköld, D., Reinius, B. \& Sandberg, R. Single-Cell RNA-Seq Reveals Dynamic, Random Monoallelic Gene Expression in Mammalian Cells. Science 343, 193-196 (2014).

71. Anders, S., Pyl, P.T. \& Huber, W. HTSeq-a Python framework to work with high-throughput sequencing data. Bioinformatics 31, 166-169 (2014).

72. Anders, S. \& Huber, W. Differential expression analysis for sequence count data. Genome biology 11, R106 (2010).

73. Lê, S., Josse, J. \& Husson, F. FactoMineR: An R Package for Multivariate Analysis. 2008 25, 18 (2008).

74. Gong, T. \& Szustakowski, J.D. DeconRNASeq: a statistical framework for deconvolution of heterogeneous tissue samples based on mRNA-Seq data. Bioinformatics 29, 1083-1085 (2013). 